\title{
Epigenetic biomarker and therapeutic intervention for dementia
}

\author{
Cumulative Doctoral Thesis
}

In partial fulfillment of the requirements for the degree

"Doctor rerum naturalium"

In the Neuroscience Program

at the Georg-August-University Göttingen, Faculty of Biology

submitted by

Md Rezaul Islam

\author{
born in \\ Bagerhat, Bangladesh
}

Göttingen, 2019






\section{PhD Thesis Committee:}

1. Prof. Dr. André Fischer, Deutsches Zentrum für Neurodegenerative Erkrankungen (DZNE), Göttingen.

2. Prof. Dr. Tiago Fleming Outeiro, University Medical Center, Göttingen.

3. Dr. Camin Dean, European Neuroscience Institute, Göttingen

Extended Thesis Committee:

- Prof. Dr. Gregor Eichele, Max Planck Institute for Biophysical Chemistry, Göttingen.

- Prof. Dr. Ralf Heinrichh, Schwann-Schleiden Research Center, Göttingen

- Prof. Dr. Susann Boretius, German Primate Center, Göttingen.

Date of oral examination: $30^{\text {th }}$ October, 2019 


\section{Declaration}

I herewith declare that I have prepared the dissertation "Epigenetic biomarker and therapeutic intervention for dementia" entirely by myself with no other aids or sources than quoted.

Göttingen, 31.08.2019

Md Rezaul Islam 
I would like to dedicate this work to my parents... 


\section{Table of Contents}

Introduction....................................

Memory...........................................

Implicit memory .........................................

Explicit memory......................................... 4

Heart Failure and memory............................5

Aging and memory $\ldots \ldots \ldots \ldots \ldots \ldots \ldots \ldots \ldots \ldots \ldots \ldots$

Alzheimer's Disease................................... 7

Histone modification and memory $\ldots \ldots \ldots \ldots \ldots \ldots \ldots . . . . . .9$

microRNAs and memory $\ldots \ldots \ldots \ldots \ldots \ldots \ldots \ldots \ldots \ldots$

microRNAs as biomarker and therapy .................12

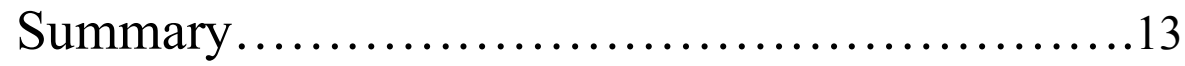

Research Article 1.............................14-61

Research Article 2............................62-107

Discussion.....................................108-112

References....................................113-119

Appendix...................................120

Acknowledgements...........................121

Curriculum Vitae...............................122-123 


\section{Introduction}

\section{Memory}

Memory is an integral part of us. It shapes our understanding and connects us with the world. Without memory, our life will be meaningless. Various brain regions, including the hippocampus, cortex, cerebellum, striatum, the amygdala can take part in the acquisition, storage, and retrieval of memory. Memory can be broadly classified into two types: a) implicit (non-declarative) memory for skill and motor functions that require reflexes and b) explicit (declarative) memory for facts, people, and events.

Little was known about how implicit and explicit memory are formed, and stored until the 1960 's. Later, many of the current understandings of memory in molecular and cellular level evolved from studies investigating Aplysia, a simple invertebrate model. Aplysia has only 20,000 nerve cells compared to a trillion in the mammalian brain ${ }^{1}$. Molecular mechanisms of learning and memory in Aplysia were later found to be conserved, at least in part, in mammals.

\section{Implicit memory}

Aplysia displays a defensive gill-withdrawal reflex after light touch to the siphon. By applying a strong stimulus to its tail (tail shock), the withdrawal reflex of both gill and siphon can be enhanced, a process that is known as sensitization of the gill-withdrawal reflex (Fig T1).
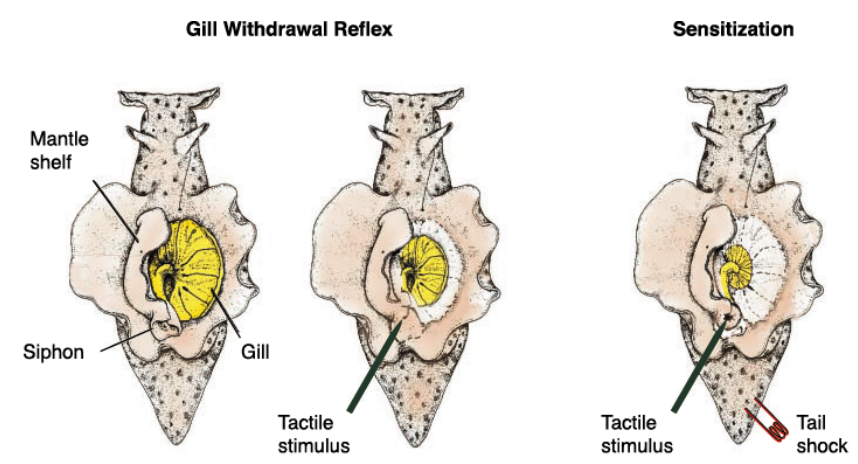

Fig T1. A simple learned behavior in Aplysia. Application of mild touch to Aplysia's siphon leads to withdrawal its gill. This reflex is further strengthened after applying a tail shock. Figure adapted from Kandel ${ }^{1}$.

During sensitization, the stimulus on the modulatory neurons from tails leads to release serotonin onto sensory neuron of the siphon ${ }^{2,3}$. Serotonin, by binding to a transmembrane receptor on pre-synaptic compartment activates Adelylyl cylase, which converts ATP to cAMP (Fig T2). cAMP activates protein kinase A (PKA) at pre-synaptic terminals leading to enhanced availability and release of glutamate, a neurotransmitter, into synaptic cleft ${ }^{5,6}$. If the actual 
stimulus (tail shock) is preceded by conditioned stimulus (stimulation of siphon) ${ }^{7}$, the synaptic strength is further increased and thus there will be a stronger gill-withdrawal reflex. This short time synaptic strength involves covalent modifications of preexisting proteins but no new protein synthesis ${ }^{1,8-10}$.

Repeated, spaced stimuli produce synaptic strength that can stay longer than that from single stimuli, and thereby forms long term implicit memory. The difference between long-term and short-term memory is that long-term memory requires new protein synthesis, and it requires post-synaptic depolarization ${ }^{11}$. During long term memory formation, serotonin-induced cAMP and PKA persists. Protein kinase A, in turn, activates mitogen-activated protein kinase (MAPK) and both move to the nucleus and then phosphorylates transcription factor CREB-1 and thereby, activates it ${ }^{12}$. CREB-1 can initiate gene expression changes in two phases: a) the first wave of immediate-early genes expression and $b$ ) the second wave of gene expression by inducing enhancer-binding transcription factor, $\mathrm{C} / \mathrm{EBP}^{13,14}$. Newly synthesized mRNAs are then delivered to specific synapses, whose stimulation initially triggered CREB-1 dependent gene expression. This phenomenon is termed as "synaptic tagging", and that is newly synthesized molecules will be delivered throughout the cell but will be only used in active synapses ${ }^{15,16}$. Transported mRNAs are translated into protein locally at the synapse ${ }^{17}$. Increased activity of PKA and local protein synthesis mark active synapses ${ }^{16}$. Of note, local protein synthesis is essential for maintaining long term memory as well. Subsequent studies revealed that the function of CREB-2 could inhibit the function of CREB-1, a memory suppressor gene, and through microRNAs (e.g., miR-124) ${ }^{18-20}$. Thus, memory formation is under tight regulation and memory suppressor genes providing a checkpoint for memory storage. However, increased serotonin levels could alleviate these suppressions. 
(a) Early implicit memory

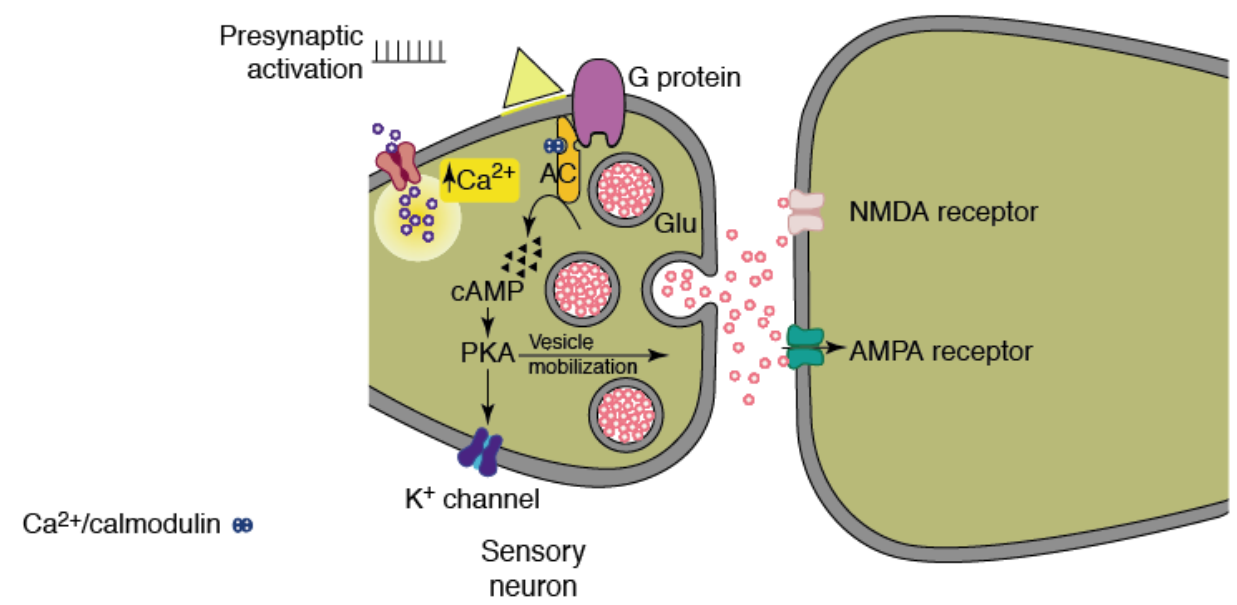

(b) Late implicit memory

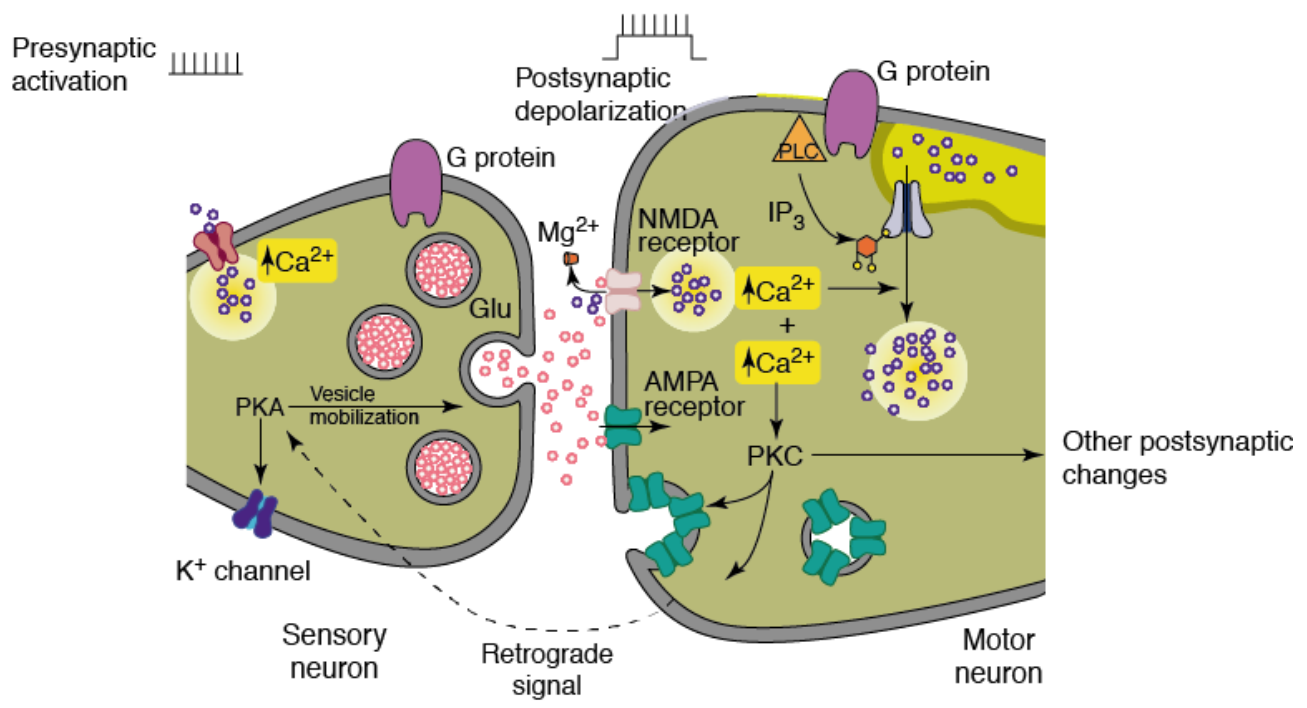

Fig T2. Signaling cascade that underlies early and late implicit memory in Aplysia.

a) Stimulus dependent pre-synaptic activation leads to increased intracellular $\mathrm{Ca}^{2+}$ concentration. $\mathrm{Ca}^{2+}$, in turn, activates adenlyl cyclase enzyme produce cAMP that triggers PKA mediated vesicle movement and neurotransmitter release into synaptic cleft. (b) In the presence of post-synaptic depolarization, along with presynaptic changes, post-synapic NMDA and PLC receptors become activated. As a result, increased $\mathrm{Ca}^{2+}$ influx through NMDA receptor and $\mathrm{Ca}^{2+}$ release from cellular reservoirs increase $\mathrm{Ca}^{2+}$ within post-synaptic compartment. $\mathrm{Ca}^{2+}$, in turn, initiates several cellular signaling processes including recruitment of AMPA receptors on the postsynaptic membrane. Moreover, glutamate released from post-synaptic compartment can act as retrograde signaling molecule to allow persistent cellular changes in pre-synaptic compartment. Figure modified from Roberts and Glanzman ${ }^{11}$.

Long term facilitation of implicit memory could be destabilized by protein degradation and later could be re-stabilized by protein synthesis at the same synaps $\mathrm{e}^{21}$. Glutamate induced postsynaptic activation of NMDA receptors and Phospholipase C (PLC) during long term implicit memory leads to increased intracellular $\mathrm{Ca}^{2+}$, that in turn, recruits AMPA receptors on the postsynaptic membrane via PKC (Fig T2) and release of glutamate as retrograde signaling molecule to trigger persistent pre-synaptic changes ${ }^{11}$. Similar mechanisms of implicit memory are found in the mammalian brain by classical fear conditioning experiments in animals. 


\section{Explicit memory}

Unlike implicit memory, explicit memory in mammalian brain involves hippocampaldependent tasks. Like implicit memory, explicit memory has a short-term phase (lasts only 1 to 3 hours) that is independent of protein synthesis and a long-term phase that requires protein synthesis. There are two types of brain cells, a) place cells in the hippocampus and b) grid cells in the entorhinal cortex, help in making navigational maps of the environment, and encode spatial information in the brain. Terje Lomo and Tim bliss first discovered activity dependent plasticity in hippocampus ${ }^{22}$, now known as long term potentiation (LTP). long-term potentiation (LTP) plays a significant role in memory in the mammalian brain. The first direct evidence regarding the role of LTP in hippocampal memory came through pharmacological interventions. Morris et al. used NMDA antagonist to block post-synaptic NMDA receptors in the rat's brain and tested their spatial memory in the water maze ${ }^{23}$. Animals with inhibition of NMDA receptors displayed spatial navigation deficits. Subsequent studies knocked out NMDA receptors specifically at various brain regions that led to different responses in mice. For example, transgenic mice with a knockdown at CA1 displayed spatial learning and memory deficits, whereas with a knockdown at CA3 and DG regions mice exhibited deficits in pattern completion, and pattern discrimination, respectively ${ }^{24-26}$.

NMDA receptor resides only on the post-synaptic terminal of a neuron. To be active, the postsynaptic terminal has to be depolarized and glutamate has to be released from the opposed presynaptic part. Thus, the activation of NMDA receptor marks active synapses. Once activated, NMDA receptors can increase $\mathrm{Ca}^{2+}$ influx, and initiate several molecular signaling pathways leading to the induction of LTP. There are two phases of LTP. The early phase involves activation of cAMP, leading to the recruitment of new AMPA type glutamate receptors and thus accompany strengthened response. The late phase, by contrast, requires both gene expression and translation ${ }^{16}$. In terms of molecular mechanisms, late-phase LTP is similar to long term implicit memory in Aplysia. Therefore, it involves PKA, MAPK signaling pathways, CREB-1 mediated gene transcription, distribution of newly synthesized proteins according to synaptic tagging hypothesis. Moreover, the late phase is associated with NMDA dependent dendritic spines enlargement and structural changes in the synapse. However, LTP induction can also be NMDA independent. For example, in CA3 neurons, LTP at mossy fiber synapse is solely dependent on the pre-synaptic neurotransmitter, and thus, post-synaptic NMDA independent ${ }^{27,28}$. Moreover, induction of LTP can vary across different brain regions and also 
within the same region with different patterns of stimuli. For instance, $100 \mathrm{~Hz}$ stimulation in the hippocampus induces LTP that is dependent on NMDA receptor activation, but $200 \mathrm{~Hz}$ stimulation-induced LTP requires voltage-gated $\mathrm{Ca}^{2+}$ channels ${ }^{16}$.

Prolonged synaptic stimulation at low frequency can lead to NMDA dependent long-term depression (LTD). Moreover, pre-synaptic activity produced shorty after post-synaptic action potentials also leads to LTD. Consequently, $\mathrm{Ca}^{2+}$ dependent phosphatases are recruited, leading to a reduction in AMPA receptors at post-synaptic compartment ${ }^{29}$. In a recent study ${ }^{30}$, Camin Dean and her colleagues showed that Synaptotagmin 3 (Syt3) could mediate AMPA receptor endocytosis, and therefore, Syt3 expression might underlie forgetting. Indeed, Syt3 knockout mice could not forget a learned task in the water maze.

Although forgetting is as essential as the formation of memory in physiological condition, a decline in both during dementia leads to disturbances in daily performances. According to the World Health Organization (WHO), worldwide, around 50 million people have dementia. Major risk factors of dementia include cardiovascular factors and aging.

\section{Heart failure and memory}

Multiple feedback mechanisms connect the heart and brain, and these two vital organs share several pathophysiological mechanisms. Both organs are affected by common risk factors including impaired metabolism, age, gender, and educational status ${ }^{31-33}$. Therefore, heart and brain diseases often co-exist in patients ${ }^{34,35}$.

Cardiac dysfunctions affect blood perfusion to the brain. At high blood pressure, the brain vascular system can maintain normal perfusion. However, at low blood pressure, regional hypoperfusion can occur. Cardiac failure leads to chronic hypoperfusion in the brain. Regional perfusion in brain is monitored by "neuro-vascular unit" that is composed of different cell types, including neurons, glia, and pericytes ${ }^{36}$. Therefore, regional hypoperfusion in chronic heart failure may accompany changes in these cell types. Structural and metabolic abnormalities along with a functional decline in multiple brain regions have been observed in patients with heart failure ${ }^{37-41}$. For example, reduced blood flow in bilateral areas of the prefrontal cortex, hippocampus, and thalamus have been reported in patients with heart failure. Patients with heart 
failure have been diagnosed with structural changes in different brain regions, including grey and white matter ${ }^{38}$. Consistently, reduced cortical thickness and extensive tissue loss in hippocampus $^{38}$ (Fig T3) and cortex ${ }^{40}$ are observed in patients with heart failure.

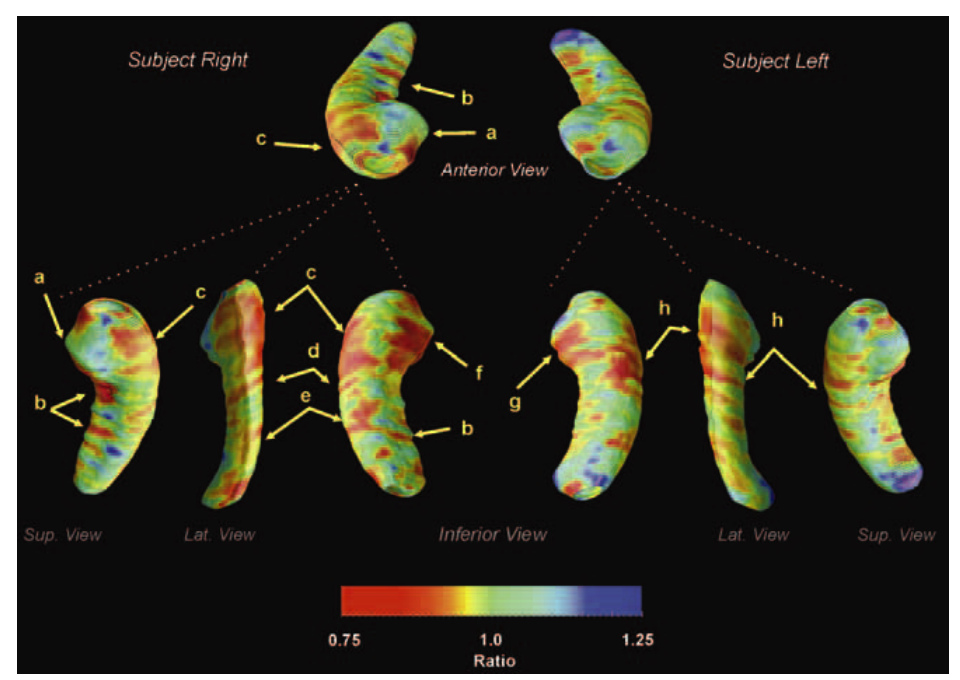

Fig T3. Regional hippocampal damage in heart failure patients.

Figure is highlighting the magnitude of hippocampal atrophy in heart failure patients. The right hippocampus (a-f) demonstrates more atrophy than the left ( $\mathrm{g}$ h). Color is representing the severity of atrophy in HF patients compared to controls. Red and orange color represent more severity of local tissue volume loss. Figure adapted from Woo et al. ${ }^{38}$

In line with structural changes in the brain, recent epidemiological studies reported a cognitive decline in heart failure patients ${ }^{42-46}$. Most of the patients are diagnosed with mild cognitive impairment, whereas some suffer from moderate to severe dementia. Patients with failing heart experience deficits in multiple cognitive domains, including executive function, attention, processing speed, visual spatial functions, leaning, and working memory ${ }^{46}$. Treatment for dementia in heart failure patients has been empirical ${ }^{44}$. Targeted and knowledge-based effective therapeutic invention is urgently required to improve cognition in heart failure patients ${ }^{44}$.

\section{Aging and memory}

Although aging is a significant risk factor for dementia ${ }^{47-50}$, it is difficult to separate the cognitively healthy aging from that of the pathological condition. Based on several cross-sectional and longitudinal data ${ }^{51}$, it is now evident that some aspect of cognition like processing speed starts declining at the early 30 s of healthy individuals. Other cognitive domains, including working memory, episodic memory decline after 55 years of age (Fig T4).

The effect of aging on memory is highly reproducible in animals. Age-associated hippocampaldependent memory deficits and reduced synaptic plasticity have been reported in several animal studies ${ }^{53-55}$. 

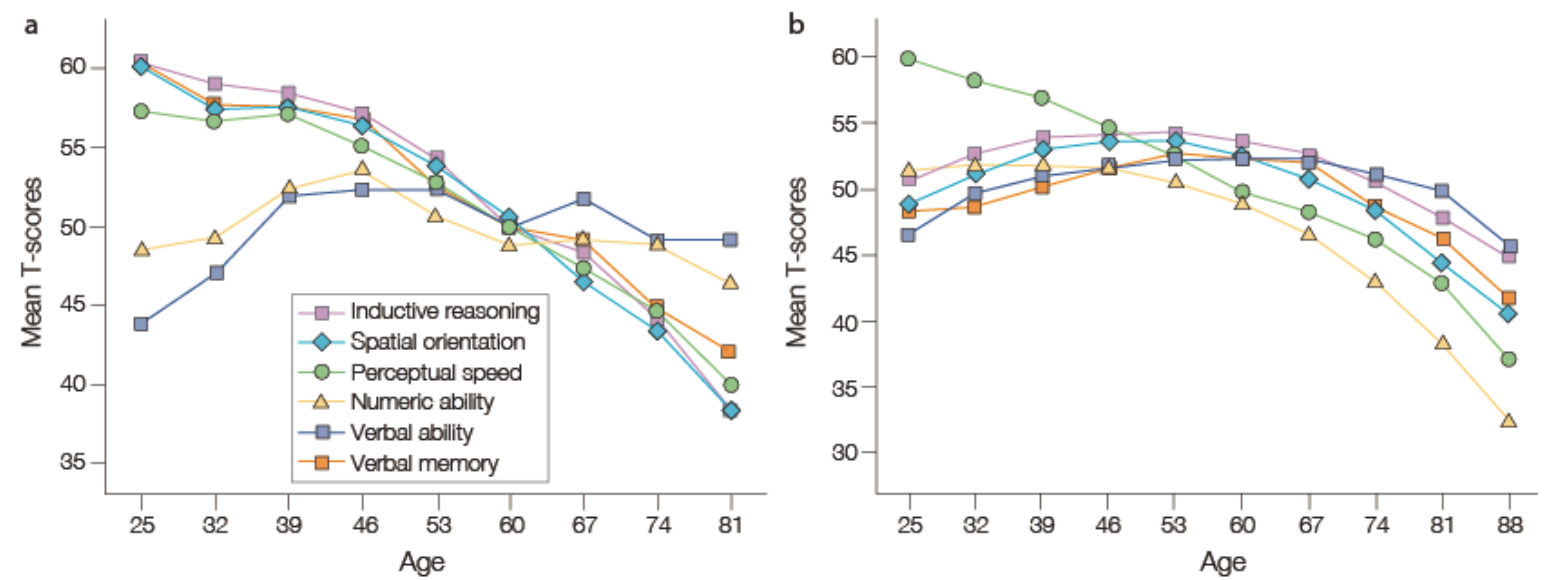

Fig T4. Timeline for age-related changes in cognition.

(a) Cross-sectional data are showing a decline in all cognitive domains after 55 years except numeric and verbal ability.

(b) Longitudinal data illustrating a decline in all domains after 55 years. Figure adapted from Hedden T and Gabrieli, J.D ${ }^{52}$

At the cellular level, aging alters several biological processes and pathways, including insulin signaling, TOR signaling, mitochondrial function, autophagy, and stress responses. While, insulin and IGF-1 can promote learning and memory in humans and animal models ${ }^{56}$, its reduction along with aging has been conserved in worms, flies, and mammals ${ }^{57}$. Mitochondrial function and TOR signaling pathways also play a critical role in physiological aging and maintaining cognition ${ }^{58-62}$. Deficits in mitochondrial function causes decreased lifespan. Decreased signaling of TOR pathways leads to increased autophagy and decreased protein translation. Altered autophagy and TOR signaling pathways have been linked to Alzheimer's disease ${ }^{63}$, and memory ${ }^{64}$. Genome-wide transcriptome analysis revealed that genes involved in stress, mitochondrial energy metabolism, neural plasticity/synaptic function, inhibitory interneuron function, ubiquitin-proteasome pathway, immune response, metal ion homeostasis, myelin, and glial pathways are mostly affected during aging ${ }^{58,65,66}$.

\section{Alzheimer's Disease}

Alzheimer's disease (AD) is an age-related neurodegenerative disease and the most common form of dementia with increasing prevalence ${ }^{67}$. At the structural level, patients display distinct brain atrophy. Microscopic changes in AD patients include extracellular deposition of Amyloid- $\beta$ (A $\beta$ ) plaques and intracellular neurofibrillary tangles (NFTs) from misfolded Tau (Fig T5), a microtubule-associated protein. AD is associated with severe neuronal loss and increased gliosis that accompany irreversible memory decline, disorientation, and confusion in patients ${ }^{68}$. While deposition of $A \beta$ plaques in $A D$ patients starts in prefrontal brain regions followed by spreading over the cortex, the NFTs show a 
highly specific spreading pattern ${ }^{69}$. In general, deposition of the tangles happens starts at the transentorhinal cortex (Braak stage I). At stage II it spreads to the entorhinal cortex and Ammon's horn in the hippocampus. At stage III, the rest of the hippocampal regions, amygdala, and the anterodorsal thalamic nuclei are affected. Finally, tau tangles spreading over the cerebral cortex leading to atrophy ${ }^{70}$ that mark stages IV-VI of the disease.

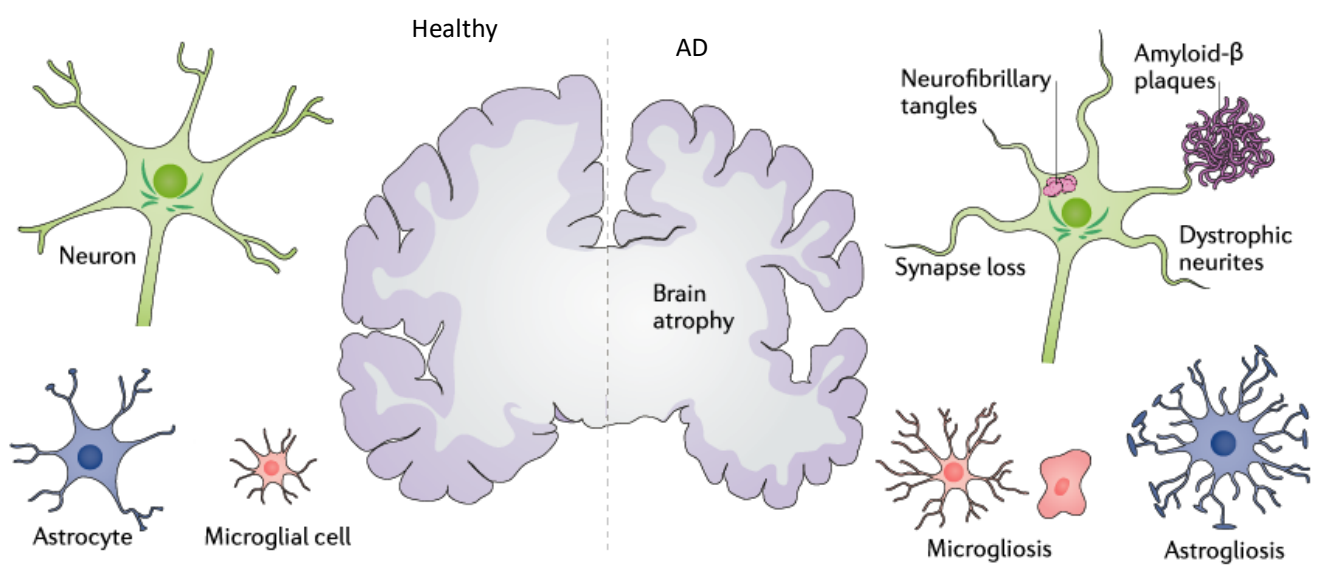

Fig T5. Pathological hallmarks of Alzheimer's disease.

Brain atrophy in Alzheimer's disease (AD) is associated with the loss of brain cells. Deposition of extracellular amyloid$\beta$ plaques and intracellular neurofibrillary tangles is linked to loss of neurites, synapse, and increased gliosis. Increased inflammatory response via microglia is also observed in $\mathrm{AD}$ pathology. Figure adapted from Congdon EE et al. ${ }^{71}$

Evidence shows that the progression of $\mathrm{AD}$ is slow, and changes in brain function take place years before the onset of dementia ${ }^{72,73}$. At the pre-clinical phase, early neuronal and metabolic changes happen in the hippocampus and entorhinal cortex, the regions that also harbor place and grid cells, essential for spatial navigation. Increased neuronal loss leading to volumetric decline contributes to disturbances in both episodic and semantic memory at mild cognitive impairment (Fig T6). In the early $\mathrm{AD}$ phase, neuronal loss continues in various brain regions, including the prefrontal cortex, the brain region that is involved in executive functioning and memory retrieval.

Clinical trials for all the drugs tested so far for $\mathrm{AD}$ have been failed ${ }^{74}$. Aducanumab, from Biogen and Eisai, is the last addition to this list as the biotech company announced in 2019 that it would discontinue phase 3 trials. Failure to accurately detect AD pathology at an early stage is one of the significant reasons why drugs have not been successful. Early accurate detection of AD is crucial and might allow applying early pharmacological interventions to stop or delay disease progression. We will need useful biomarkers to diagnose incipient patients at the pre-clinical stage. 



Fig T6. Progressive changes in AD brain.

a) Early changes in the hippocampus and the entorhinal cortex may affect spatial navigation at pre-clinical AD.

b) increased neuronal loss accompany volumetric loss in the medial temporal and parietal lobes of mild cognitively impaired AD patients.

c) rest of the medial temporal lobe, and frontal lobe are affected at an early AD. Spatial navigation is severely affected at an early AD. Figure adapted from Coughlan et al. ${ }^{75}$

\section{Histone modification and memory}

A long stretch of DNA is packaged into the microscopic nucleus in the form of chromatin. 147 base pairs long DNA is wrapped around a histone octamer to form a nucleosome, the core unit of chromatin ${ }^{76}$. Subsequently, chromatin adopts a high order organization to position itself within the nucleus. All Histones H2A, H2B, H3, H4 in nucleosomes can be post-translationally modified, mostly at different amino acid residues on their tails. Various modification marks on histones determine whether chromatin will be transcriptionally active (euchromatin) or inactive (heterochromatin). Active chromatin will adopt a relaxed and open state allowing non-histone transcription factors and enzymes to interact with DNA. Interactions between different histone modifications and DNA can determine the fate of a gene. These histone-DNA interactions can happen at the promoter, gene-body, and enhancer of a gene. A transcriptionally active gene often has $\mathrm{H} 3 \mathrm{~K} 4 \mathrm{me} 3, \mathrm{H} 3 \mathrm{~K} 4 \mathrm{me} 2$, and $\mathrm{H} 3 \mathrm{~K} 9 \mathrm{ac}$ modifications at its promoter, whereas H3K27me3, H3K9me3 will mark an inactive gene promoter ${ }^{77}$. Some genes are only temporally expressed, therefore are termed as poised. These genes have both an active (H3K4me3) and an inhibitory mark (H3K27me3) at their promoters ${ }^{77}$. Histone modification at gene body (e.g., H3K36me3) can affect the splicing of a given gene ${ }^{78}$. 
Different histone mark readers can recognize the existing histone modification mark; writers can place marks and erasers can remove histone marks to shape distinct expression patterns ${ }^{76}$. For example, $\mathrm{Kmt} 2 \mathrm{a}$ is a methyltransferase that will add methylation on Histone 3 lysine 4 residue leading to gene activation, whereas HDAC is a histone deacetylase, that can erase acetyl group from Histone 3 lysing 9 residue and thus inactivates gene expression. Inhibitors of HDAC [e.g., Vorinostat (SAHA)] can inhibit the function of the HDAC and alter gene expression ${ }^{79}$.

Histone modifications can change upon different environmental stimuli and play a key role in memory formation. In one of the first experiments to link histone modification with memory, Fischer et al. investigated the effects of environmental enrichment on histone modifications ${ }^{80}$. Authors showed that environmental enrichment increased histone acetylation in mice brain and could restore the memory of the memory-impaired mice. By using HDAC inhibitors (e.g., sodium butyrate and TSA), the cognitive benefits from increased acetylation could be reproduced ${ }^{80}$. Besides acetylation, changes in histone methylation have been linked to memory. Gupta et al. reported that increased $\mathrm{H} 3 \mathrm{~K} 4 \mathrm{me} 3$ at the promoter of memory-related genes happen after $1 \mathrm{~h}$ of associative learning ${ }^{81}$. Moreover, recent studies revealing that modulation of different histone-modifying enzymes (e.g., methyl and acetyl transferases) can affect memory as well ${ }^{82-84}$.

\section{microRNAs and memory}

microRNAs are $\sim 19-22$ base long small nuclear RNAs that regulate target gene expression at the posttranscriptional level in healthy and disease conditions ${ }^{85,86}$. Their key roles include cell differentiation and signaling, development, and pathogenesis of many neurological disorders ${ }^{87,88}$. The biogenesis of microRNA has been conserved in all mammals ${ }^{89}$. Both RNA polymerase II (Pol II) and III (Pol III) can synthesize primary microRNA transcript (pri-microRNA) in the nucleus (Fig T7). Once primicroRNA is synthesized, it is then cleaved by the Drosha-DGCR8 (Pasha) microprocessor complex and produces precursor microRNA (pre-microRNA). Subsequently, pre-microRNA is exported from the nucleus to the cytoplasm by Exportin-5-Ran-GTP (Fig T7). In the cytoplasm, an enzyme named Dicer cleaves the pre-microRNA hairpin to produce double-stranded mature microRNA. One of these strands remains functional as it is guided toward its target mRNA by Argonaute (Ago2) proteins and by binding to its 3' UTR region can silence target mRNA expression. Depending on its full or partial complementary sequences, silencing could be achieved through one of the processes from mRNA cleavage, translational repression, or de-adenylation (Fig T7). The other strand of the mature 
microRNA is called the passenger strand, and later degraded. Of note, one microRNA can target multiple mRNAs, and in turn, one mRNA can be targeted by more than one microRNA, giving rise to a complex regulatory network of gene expression and protein homeostasis.

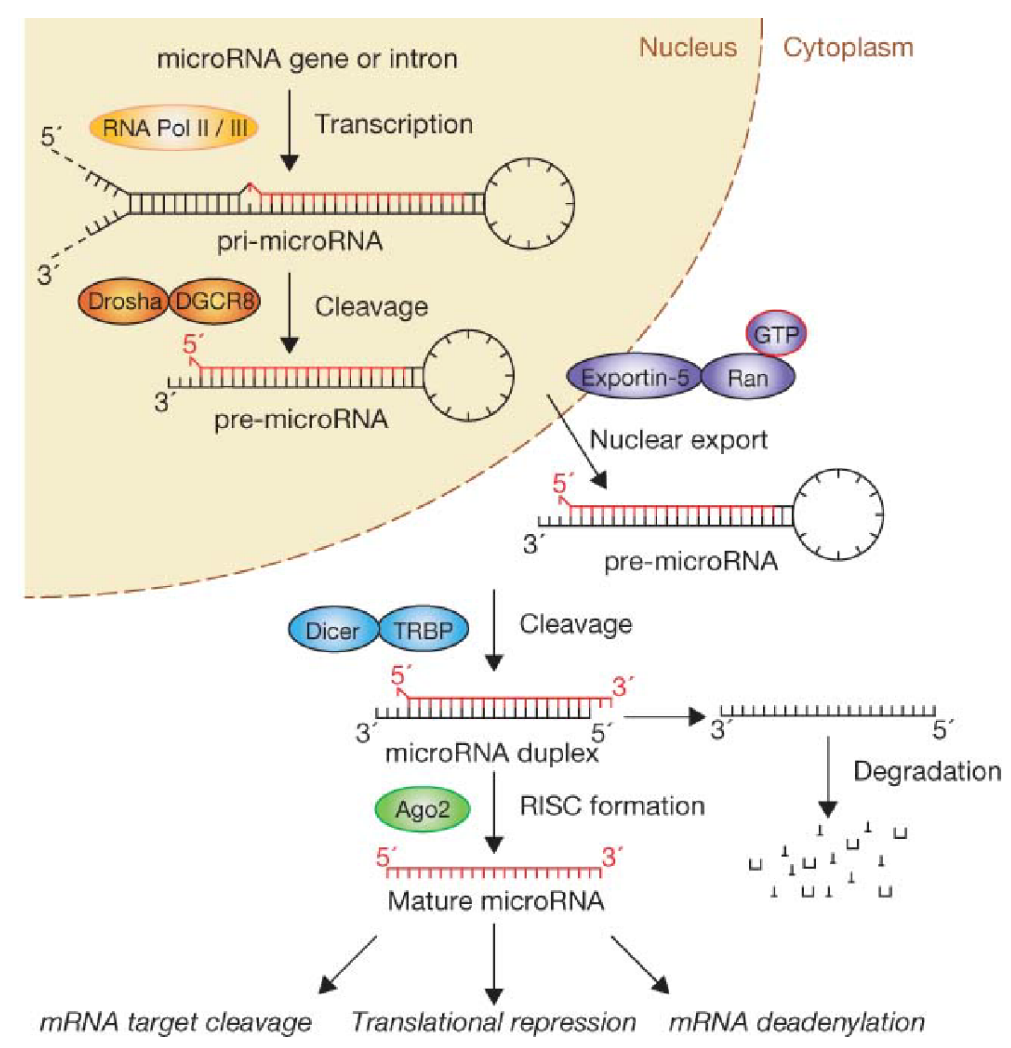

Fig T7. microRNA biogenesis.

Precursor microRNAs (pre-microRNAs) are transcribed in the nucleus. Pre-microRNAs are transported to cytoplasm via a transporter called Exportin 5. Dicer removes the hairpin from the pre-microRNA to produce double-stranded maturemicroRNAs. One of the strands is guided towards the site of action by the RISC complex. The other strand is called the passenger strand, and it is degraded. At the target site, microRNAs can repress the target genes expression by target mRNAs cleavage, translation repression, and target mRNA de-adenylation. Notably, one microRNA can regulate multiple target genes. Figure adapted from Winter J et al. ${ }^{90}$

Rajasethupathy et al. showed that one microRNA, miR-124 could inhibit the function of CREB-1 transcription factor leading to reduced synaptic plasticity and memory in Aplysia ${ }^{91}$. Later, microRNA-mediated regulation of learning and memory was also reported in Drosophila ${ }^{92}$. Subsequently, the microRNA processing enzyme, Dicer, was linked to learning and memory ${ }^{93}$. Cell type-specific knockout of Dicer enhanced synaptic plasticity and memory in a variety of learning tasks, including spatial learning in the Morris water maze, and contextual and trace fear conditioning. 
These effects were accompanied by decreased expression of several microRNAs, including miR-125b and miR-132, which were previously shown to be expressed locally and regulate dendritic spine formation ${ }^{94}$. A recent study reported that miR-34c might play a role in memory impairment ${ }^{95}$. Authors have observed an increased level of miR-34c in the hippocampus of 24 months old mice, a model for age-associated memory impairment. This increased level of the miR-34c was correlated with impaired memory function in the water maze test, and functionally could repress the Sirt1 protein expression. Another study showed that increased Sirt 1 enhances dendritic spine density and synaptic plasticity in the hippocampus through the down-regulation of miR-134 ${ }^{96}$. Thus, several microRNAs can crosstalk with the same mRNA or different mRNAs in a gene regulatory network and can play a key role in fine-tuning gene expression patterns in response to learning and memory.

\section{microRNAs as biomarker and therapy}

microRNAs have the potentials to be biomarkers for several reasons. For example, they are highly conserved among species, extremely stable in different experimental conditions, and key gene regulatory elements in the biological system. Moreover, the systemic circulation of microRNAs enables them to take part in cell-to-cell communication and signaling in normal biological processes ${ }^{97}$ as hormone does ${ }^{98}$. Additionally, microRNA levels from biological fluids can be quantified easily in limited experimental settings. In line with this, recent studies have shown that subsets of microRNAs may have clinical relevance as biomarkers to diagnose a particular stage, and progression of the disease $^{99-101}$. Measuring the microRNAome in the blood is discussed as a promising strategy to develop biomarkers for brain ${ }^{102}$ and other human diseases ${ }^{103}$. Despite its high promise, effective microRNA-based biomarker to diagnose incipient dementia is still missing.

A number of studies revealed therapeutic potentials of microRNAs ${ }^{104-107}$. Recent FDA approval of first small RNA based (application number: 210922) in 2018 is one step forward towards microRNAbased therapeutics. More biotech companies (e.g., Miragen, Regulus Therapeutics, Synlogic) are now investing in the development of microRNA-related drugs. Several drugs related to different microRNAs including MRX34 (miR-34), MRG 110 (miR-92), MesomiR-1 (miR-16), Miravirsen (miR-122), MRG-201 (miR-29), RG-012 (miR-21), MRG-106 (miR-155) are already in clinical trials $^{108}$. Although the drugs mentioned above are being tested in mostly cancer treatment, microRNAbased drug to improve cognition in dementia remain underexplored. 


\section{Summary}

\section{Summary}

Dementia is the most significant health challenge in modern times, currently affecting over 50 million people worldwide. The number of people afflicted with this disorder is on the rise and expected to double by 2025. Causative and symptomatic treatments of this disease have been so far unsuccessful. This is partly due to the fact that patients are only diagnosed with dementia at an advanced stage, when a massive neuronal loss has already manifested. There is substantial evidence memory impairment is preceded by molecular changes that happen years before the onset of cognitive decline. Therefore, there is an urgent need for biomarkers to diagnose early changes in the brain, which would allow clinicians to intervene therapeutically at an earlier phase of the disease. Moreover, it is equally important to study the underlying mechanisms of risk factors that might precipitate these early changes in the brain. Management of those risk factors may significantly reduce the progression of dementia or even prevent the onset of the disease.

Therefore, in this cumulative thesis, I first tested the hypothesis that microRNAs in blood could be potential early diagnostic biomarkers for dementia. By using human cross-sectional and mouse longitudinal data, coupled with advanced multi-step systems biology approach, I report a blood-based "microRNA signature" that can inform about early cognitive decline in healthy and pathological conditions. Intriguingly, by manipulating the level of one of the signature microRNAs, I could improve memory in cognitively impaired mice. These data suggest that the "microRNA signature" that I report can not only be used as a diagnostic marker but also as a therapy to manage cognitive deficits in early dementia.

In addition, I investigated how cardiac failure, a risk factor of dementia, could affect brain functions at the molecular level. By using transgenic mice with failing hearts, I report down-regulation of memory-related genes in the hippocampus leads to cognitive deficits in transgenic mice. Analyses of genome-wide distribution of $\mathrm{H} 3 \mathrm{~K} 4 \mathrm{me} 3$ reveal that reduced levels of $\mathrm{H} 3 \mathrm{~K} 4 \mathrm{me} 3$ at the promoters of genes may underpin cognitive changes. Consistently, through oral administration of SAHA, an inhibitor of HDAC, I delineate that the observed cognitive deficits can be rescued. At the molecular level, SAHA could partially restore both microRNAome and RNAome, highlighting its potential as a therapeutic intervention to ameliorate cognitive deficits following heart failure.

Both of these studies provide key insight to molecular underpinnings and therapeutic interventions of early dementia. While epigenetic biomarker based on microRNAs could be useful to stratify individuals at risk of developing dementia, epigenetic drugs could be suitable strategy to restore memory and attenuate the future risk of dementia. 
Research Article 1 (prepared for submission)

\section{Circulating microRNAs as marker and therapy for early cognitive deficits}

Md Rezaul Islam ${ }^{1}$, L. Kaurani ${ }^{2 \$}$, T. Berulava ${ }^{1}$, Urs Heilbronner ${ }^{3}$, Monika Budde $^{3}$, K. Niamkovich ${ }^{3}$, T.P. Centeno $^{1}$, V. Elerdashvili ${ }^{1}$, E. Benito ${ }^{1}$, P. Rao ${ }^{2}$, C. Kerimoglu ${ }^{1}$, M. Boroomandi ${ }^{1}$, M Sadman Sakib ${ }^{1}$, Fanny Senner $^{3}$, Janos Kalman, J ${ }^{3}$, S. Burkhardt ${ }^{1}$, B. Malchow ${ }^{4}$, H. Bickeboeller ${ }^{5}$, T. Schulze ${ }^{3 *}$, P. Falkai ${ }^{*}$, F. Sananbenesi $^{6^{*}}$ A. Fischer ${ }^{1,2^{*}, \#}$ 


\section{Circulating microRNAs as marker and therapy for early cognitive deficits}

Md Rezaul Islam ${ }^{1 \$}$, L. Kaurani2 ${ }^{2}$, T. Berulava ${ }^{1}$, Urs Heilbronner ${ }^{3}$, Monika Budde $^{3}$, K. Niamkovich ${ }^{3}$, T.P. Centeno ${ }^{1}$, V. Elerdashvili ${ }^{1}$, E. Benito ${ }^{1}$, P. Rao ${ }^{2}$, C. Kerimoglu ${ }^{1}$, M. Boroomandi' ${ }^{1}$, M Sadman Sakib $^{1}$, Fanny Senner ${ }^{3}$, Janos Kalman, $J^{3}$, S. Burkhardt ${ }^{1}$, B. Malchow ${ }^{4}$, H. Bickeboeller ${ }^{5}$, T. Schulze ${ }^{3 *}$, P. Falkai ${ }^{* *}$, F. Sananbenesi ${ }^{* *}$ A. Fischer ${ }^{1,2 *, \#}$

\footnotetext{
${ }^{1}$ German Center for Neurodegenerative Diseases, Department for Epigenetics and Systems Medicine in Neurodegenerative Diseases, Von Siebold Str 3A, 37075, Göttingen, GERMANY

${ }^{2}$ University Medical Center Göttingen, Dept. For Psychiatry and Psychotherapy, Von Siebold Str. 5, 37075, Göttingen, GERMANY

${ }^{3}$ Institute of Psychiatric Phenomics and Genomics, Medical Center of the University of München, Nußbaumstr.

7, 80336, München, GERMANY

${ }^{4}$ Department of Psychiatry and Psychotherapy, Medical Center of the University of München, Nußbaumstr.

7, 80336, München, GERMANY

5 Department of Genetic Epidemiology. University Medical Center Göttingen, Humboldtallee 32, 37073, Göttingen, GERMANY

${ }^{6}$ Research Group for Genome Dynamics in Brain Diseases, Von Siebold Str. 3A, 37075, Göttingen, GERMANY

\#To whom correspondence should be addressed: Prof. Dr. Andre Fischer; andre.fischer@dzne.de $\$ *$ Equal contribution
} 


\begin{abstract}
Deregulation of cognitive function is a key pathological hallmark of various neuropsychiatric and neurodegenerative diseases. Thus, there is an urgent need for minimally invasive biomarkers that could inform about future risk of developing cognitive defects. Here, we established an experimental approach suitable for the identification of circulating microRNAs that inform about cognitive decline at the preclinical phase. We combined healthy human cross-sectional data with mouse longitudinal data and several other in house and published datasets through an unbiased feed-forward feed-backward screening approach. We report a robust "microRNA signature" of three microRNAs that inform about early cognitive deficits. We could further show that through manipulation of one of these signatures, microRNA-181a-5p, early cognitive deficits could be ameliorated in mice. We suggest that our "microRNA signature" would be very informative for the early diagnosis of dementia. While further clinical trials will be necessary to test both signature and inhibitor in a large human cohort, our data provide strong evidence about potentials of the signature as an early diagnostic marker and microRNA-181a-5p inhibitor as an effective therapeutic intervention in early dementia.
\end{abstract}

Key words: microRNA, brain plasticity, neurodegeneration, Alzheimer's disease, biomarker 


\section{Introduction}

Impaired cognitive function is a key pathological hallmark of various neuropsychiatric and neurodegenerative diseases, and in many instances, pathological changes in cognitive function increase slowly over time ${ }^{1}$. Bonafide examples are age-associated neurodegenerative diseases such as Alzheimer's disease (AD). As a result, AD patients are often only diagnosed at an advanced stage of pathology, which is considered to be a significant reason why causative treatments are so far ineffective 2,3456 . Therefore, there is a general need for a biomarker that could inform about cognitive function and help to monitor healthy individuals to detect patients at risk for developing cognitive diseases. Advances in this direction stem, for example, from innovative structural and functional brain imaging approaches ${ }^{7}$ that are however not suitable for monitoring healthy individuals in the context of routine annual check-up screenings. Therefore, an inexpensive and minimal invasive molecular biomarker that would help to enrich individuals at risk who could then undergo more detailed, cost-intensive and time-consuming examinations are urgently needed. A recent line of research indicates that circulating small non-coding RNAs could serve as diagnostic biomarkers for various disorders ${ }^{8}$, including brain diseases 91011 12 10,13-16 17 18. The best-studied small non-coding RNAs are microRNAs that are 19-22 nucleotide long RNA molecules regulating protein homeostasis via binding to a target mRNA thereby causing its degradation or inhibition of translation ${ }^{19}$. MicroRNAs are particularly interesting as potential biomarkers since changes in the microRNAome are believed to reflect subtle changes in cellular homeostasis. Moreover, microRNAs are extremely stable in cell free environments, are resistant to thaw-freeze cycles ${ }^{10}$ and have been implicated with learning and memory function and dementia $\begin{array}{lllll}20 & 21 & 22 & 23 & 24\end{array}$. Besides, microRNA based therapies were able to improve cognitive function in rodents 2526 . In this study, we aim to identify circulating microRNAs that could inform about cognitive performance at the onset of cognitive decline. We base our approach on the integrative analysis of humans and corresponding rodent models. To this end, we identify microRNAs that correlate with the interindividual variability in cognitive performance in healthy humans. Our data indicate that these microRNAs control signaling pathways linked to the aging process. We, therefore, study blood microRNAome of aging mice longitudinally. Via an integrative analysis combining both mouse and human data, we identify "microRNA signature" consisting of three microRNAs. Our circulatory signature shows increased expression at the early onset of cognitive decline in both mice and humans. Similar expression changes are also observed in CSF, plasma, and blood from humans with mild cognitive impairment. We further show that inhibition of 
microRNA-181a-5p, one of the members of the signature, provides cognitive benefits to mice. We suggest the analysis of the reported "microRNA signature" should be included in ongoing longitudinal clinical and population-based studies that measure cognitive function to evaluate the risk of developing dementia. 


\section{Results}

Asymptomatic changes in circulating microRNAs are linked to cognitive variability in young, healthy humans

Our study aims to identify circulating microRNAs that could inform about cognitive function and have potentials as an early diagnostic marker for dementia. Since preclinical molecular changes in dementia develop decades earlier ${ }^{27,28}$, a comparison between healthy and demented subjects to identify markers would be a suboptimal approach. Therefore, we decided to take advantage of the fact that cognitive abilities vary in healthy individuals ${ }^{29}$ and asked whether circulating microRNAs could correlate with these variabilities. Thus, we recruited 132 healthy individuals (age $25.95 \pm 5.1$ years) that were subjected to a battery of eight different cognitive tests to determine their executive function, working memory and intelligence (Budde et al., 2018 in press; http://www.psycourse.de). Next, we collected blood samples from all participants at the time of memory testing (Fig 1A). As blood collection approach, we employed PAXgene based method as we found out that application of this method was easiest in clinical settings and microRNAome data obtained from this method are highly reproducible and comparable between mouse and human (Fig S1). Subsequently, isolated RNA from collected blood was subjected to small RNA sequencing. After adjusting for gender effect on microRNA expression data, we carried out an unsupervised weighted co-expression clustering analysis and identified four coexpression modules (Fig 1B). Next, we calculated for each individual a composite cognitive score (weighted cognitive performance) (see Methods for detail) and asked whether the weighted cognition could correlate with identified co-expressed modules. Our analysis revealed that among four modules, two (e.g., turquoise and blue) showed a negative correlation whereas one (brown) module showed a positive correlation with cognition (Fig 1B, Table S1). Relying on the human tissue atlas for microRNAs ${ }^{30}$ we detected 17 circulating microRNAs (Table S2) from these three modules, which are also highly expressed in the brain and might, therefore, play a direct role in cognitive function (Fig 1C). 
A


B


D

\section{Biological processes}

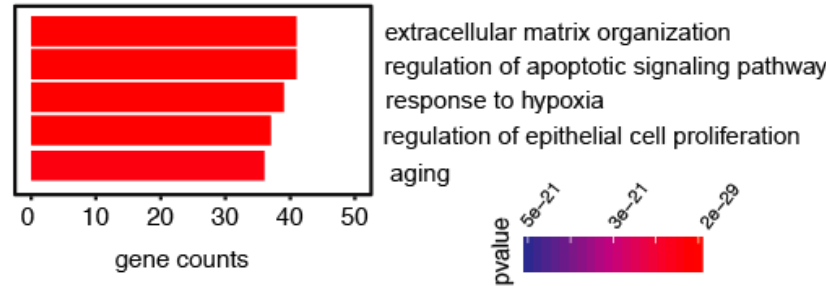

Fig 1. Analyzing circulating microRNAs in healthy human subjects.

A. Outline of the experimental approach. Healthy subjects ( 74 male and 58 female) participated in an array of cognitive tests e.g., trail making tests (part A and B), digit symbol test, digit span forward and backward test, multiple-choice vocabulary and intelligence test (MWT-B) followed by the donation of blood for molecular analysis. RNA was isolated from frozen blood and subjected to small RNA sequencing. Samples were filtered based on sequencing data quality and presence of all of the aforementioned cognitive test scores, and thus, data from 132 individuals in total were used for downstream analysis. B. Weighted co-expression analysis of all expressed microRNAs found four cluster modules. A weighted cognitive score was enumerated from various cognitive domains based on factor analysis and later used to demonstrate a correlation between phenotype and microRNA expression. Brown, blue, and turquoise cluster modules significantly (p-value $<0.05$ ) correlated with cognitive performance $(n=132$, age $25.95 \pm 5.1$ years). Brown cluster displayed a positive correlation while both blue and turquoise clusters showed negative correlations with the cognitive score. C. Heat map showing the expression of microRNAs from brown, blue and turquoise clusters that displayed a significant association ( $\mathrm{p}$ $<0.05$ ) with cognitive performance in various human organs. D. Target genes with strong experimental evidence for brain enriched microRNAs were retrieved from miRTarbase and used to perform gene ontology analysis. Featured significant (adjusted p-value $<0.05$ ) biological processes include extracellular matrix organization, regulation of cell proliferation, apoptotic signaling processes, response to hypoxia, and aging.

GO-term analysis of the confirmed mRNA targets of these microRNAs revealed that changes in their expression could affect aging (Fig 1D). These data suggest that changes 
in circulating microRNAs at a young age may lead to subtle changes in aging-related processes and could thereby contribute to cognitive variabilities.

Changes in circulating microRNAs correlate with age-associated memory decline in mice

To investigate how aging could influence cognition and microRNA expression, we carried out our further experiments in mice. Age-associated memory decline is a well-established and highly reproducible phenotype in mice $^{31}$, and both mice and humans share similarities in underlying molecular processes. To monitor longitudinal cognitive changes along with aging, we subjected mice to the water maze, a well-established test for spatial reference memory. Our decision was based on the fact that this test enables the sensitive and repeatable measure of several comparable cognitive domains in mice and in humans ${ }^{32-34}$, a feature that is critical for longitudinal studies. Although old mice are known to show memory impairment (e.g., 20 months) ${ }^{35}$, a recent cross-sectional study revealed they could display earlier impairment. For example, compared with three months, mice display early cognitive deficits at 16 months, but they lack memory impairment at 12 months ${ }^{36,37}$.
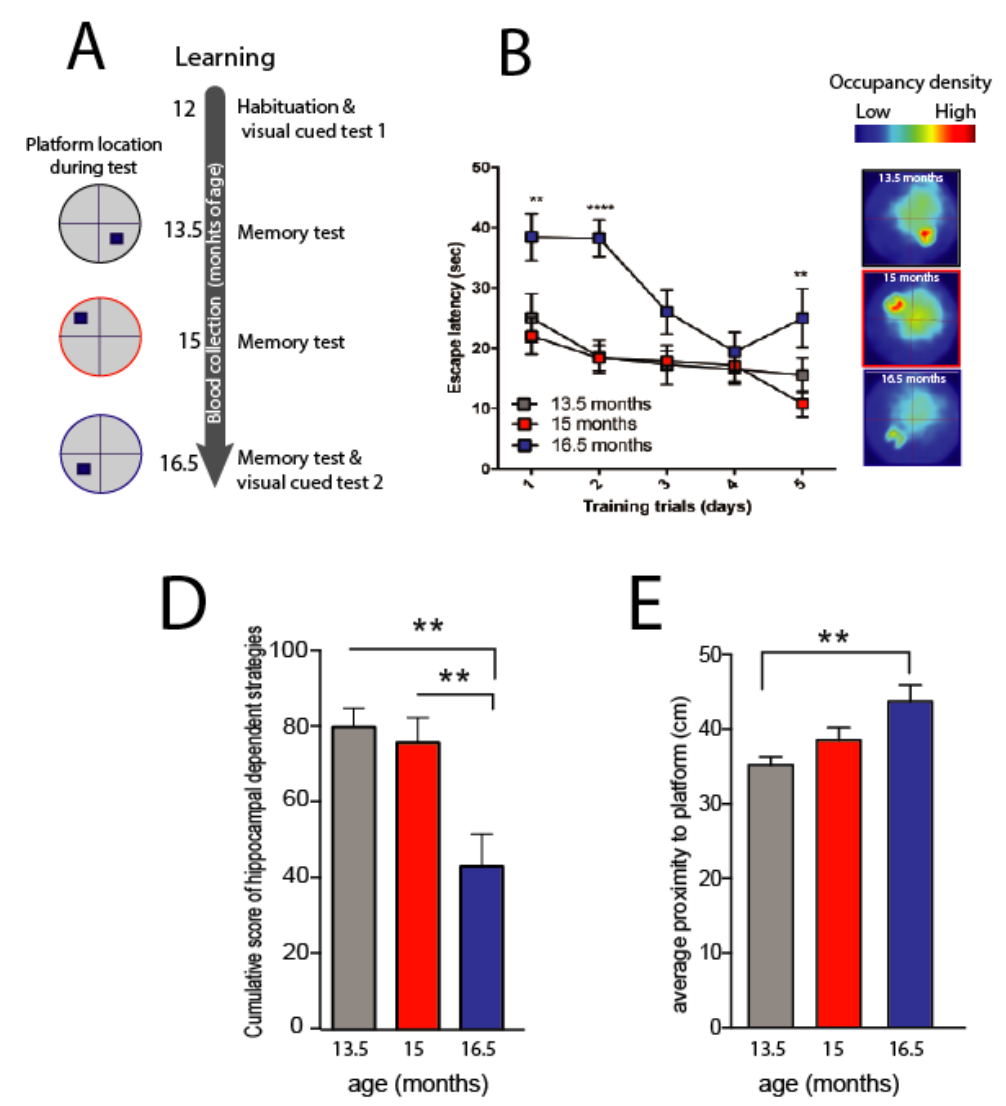
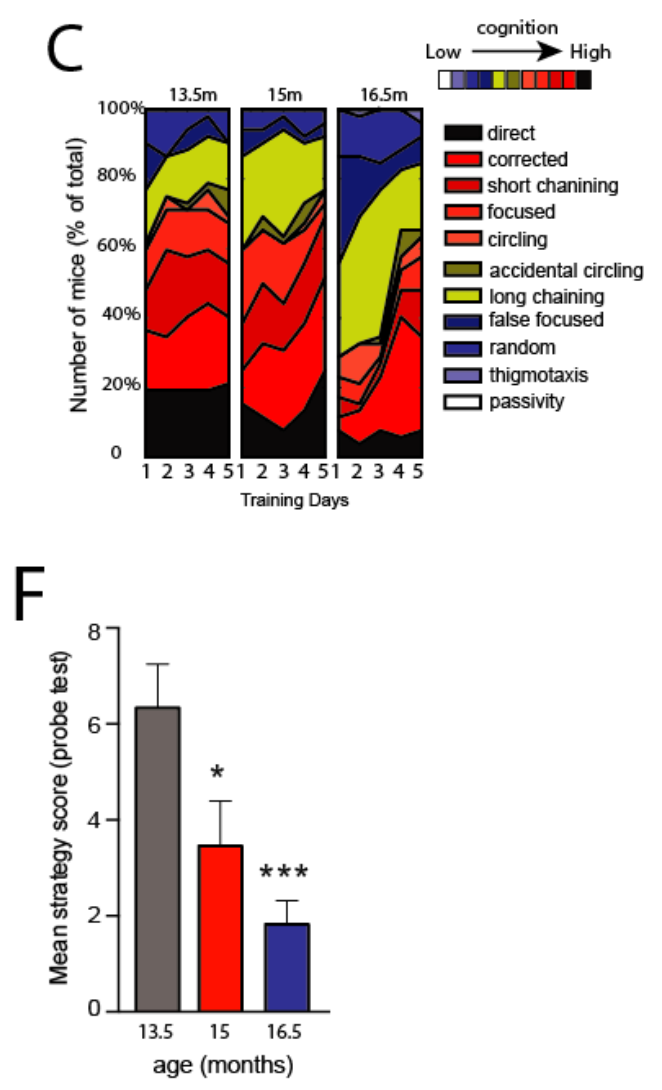

Fig 2. Age-associated memory impairment in mice in a longitudinal experiment.

A. Experimental design of the main experiment $(n=10$ each group). At 12 months of age, mice were subjected to the water-maze training protocol in order to habituate the animals to the procedure. 
Subsequently, mice were subjected to water maze training followed by a probe test at $13.5,15$, and 16.5 months of age. The platform position was altered during each training procedure. Blood was collected upon completion of each water maze procedure. A visual cued test was performed after the first and after the last blood collection when mice were 12 and 16.5 months of age, respectively. B. Left panel: Escape latency during water maze training among different age groups (Two-way ANOVA followed by Tukey's multiple comparisons, training trials: $\mathrm{p}$-value $<0.0001$; age: $\mathrm{p}$ 0.0004). On day one and two, there was a significant $(p$-value $<0.05)$ difference between mice at 13.5 vs. 16.5 months and 15 vs. 16.5 months of age. On the $5^{\text {th }}$ day of training, there was a significant difference observed between 15 and 16.5 months of age. Right panel: Density plots for grouped data showing the position of mice in correspondence to the platform during the training days. C. Analysis of the different search strategies during the water training sessions. Note that especially at 16.5 months of age mice adapted hippocampal independent search strategies indicative of impaired cognitive function. D. The cumulative score for hippocampal-dependent strategies during water maze training was significantly impaired when comparing mice at 16.5 months of age to their performance at 15 or 13.5 months of age (Ordinary One-way ANOVA, Tukey`s multiple comparison test). E-F. Performance during probe test. E. Average proximity (One-way ANOVA) and, F. Cumulative search strategy among groups (one-way ANOVA, Tukey`s multiple comparison test). ${ }^{*} \mathrm{p}<0.05,{ }^{* *} \mathrm{p}<0.01$, $* * * \mathrm{p}<0.001$. All error bars indicate mean \pm SEM.

Given that the average life span of mice is $24-26$ months $^{38}$, we reasoned that analyzing spatial learning and memory in mice from early middle age (12 months) until late middle age (16.5 months) might allow us to investigate early cognitive transition. Therefore, a group of mice $(\mathrm{n}=10)$ was subjected to spatial reference memory test at 12 months, and we repeated this every 1.5 month until mice became 16.5 months old, hence this group was termed as "learning group" (Fig 2A). We collected blood from mice at the aforementioned time points after the water maze test and importantly, repeated blood collection from orbital sinus did not affect their vision (Fig S2). Additionally, we collected blood from a group of aging mice that were not subjected to memory training. This group served as the control for effects due to water maze test, and it was named as "home-cage group" (Fig S3A). During spatial memory learning at evenly spaced time points, the location of the platform and the visual cues were randomly exchanged to examine newly formed spatial reference memories (Fig 2A). At 12 months of age, however, mice were allowed to habituate with water maze and did not perform the memory test. Therefore, we evaluated their performances in training and retrieval of reference memory at 13.5, 15, and 16.5 months. Escape latency during the training procedure - a measure of spatial reference learning ability - was significantly impaired at 16.5-months compared with that from 13.5 and 15 months (Fig 2B). Further sensitive analysis employing a modified version of the MUST-C algorithm ${ }^{32}$ revealed various spatial strategies that mice employed during training (See methods for more details). This analysis showed us that between 15 and 16.5 months of age mice adapted search strategies related to impaired cognitive ability (Fig 
2C). Consistently, at 16.5 months, mice exhibited a reduction of hippocampal-dependent "direct", "corrected", "short chaining", and "focused" search strategies (Fig 2C). In line with this, the cumulative cognitive score was significantly reduced at 16.5 months compared with that from 13.5 and 15 months of age (Fig 2D). Moreover, memory retrieval data revealed that average proximity was increased (indicates poor memory) (Fig 2E) whereas the mean strategy score (Fig 2F) were significantly reduced at 16.5 months of age compared with 13.5 months. Since all mice were able to find the platform rapidly in a visually cued test performed at 12 and 16.5 months of age (Fig S1), these data collectively show that at 16.5 months mice exhibit robust age-associated defects in both learning and memory of a spatial reference task.

Next, we isolated RNA from collected blood and subjected this RNA to small RNA sequencing. We performed this experiment in both "learning" and "home-cage" group. We employed "home-cage" as a control group to exclude microRNAs that potentially deregulate in response to water maze training. The sequencing data obtained from both learning and home-cage groups was fit to a likelihood ratio test model and adjusted for hidden confounding factors. Thus, compared to expression data from 12 months, we found differentially expressed microRNAs at 13.5, 15, and 16.5 months. We detected 69 deregulated microRNAs in the learning-group (Fig 3A), whereas 78 microRNAs were differentially expressed in the home-cage-group (Fig S3B, Table S3). Hierarchical and fuzzy clustering analysis revealed that the differentially expressed microRNAs detected in both groups fit into two main clusters: one cluster containing microRNAs that increased while the other representing microRNAs that decreased with advanced age (Fig 3B, Fig S3C). The specificity of these findings was further confirmed by the fact that many circulating microRNAs did not change during aging (Fig S3D). Next, we compared the differentially expressed microRNAs between "learning" and "home-cage" groups and identified 55 microRNAs that were similarly deregulated during aging $(r=0.99)$ in both groups (Fig S3E). These data indicate that the 55 microRNAs might reflect important homeostatic changes associated with the aging process and were named as "aging responsive" microRNAs (Table S4). Gene ontology analysis for the experimentally validated target genes of the 55 aging responsive microRNAs revealed that they might play a role in brain-specific processes, such as "modulation of synaptic transmission", 
"regulation of synaptic plasticity" and "learning" as well as pathways for apoptosis (Fig 3C-D).
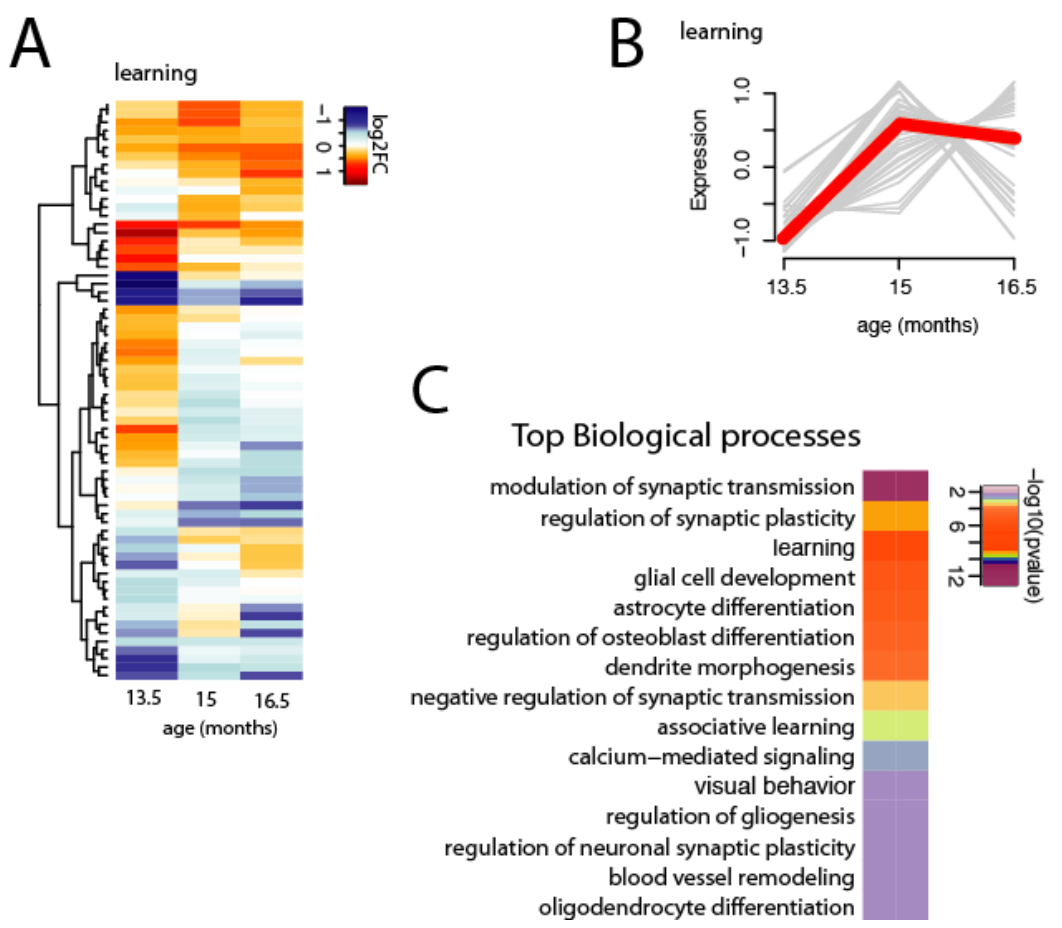

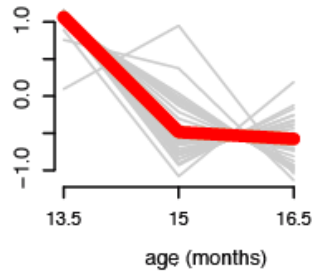

D

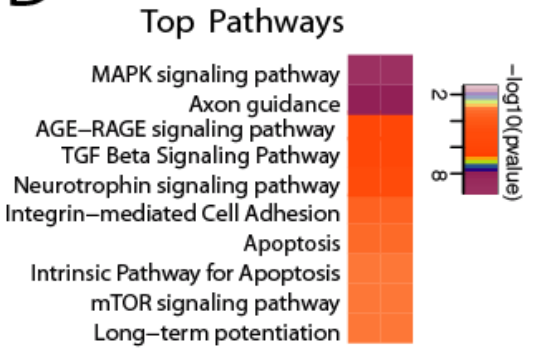

Fig 3. Age-associated changes in the circulating microRNAome reveal pathways linked to cognitive function.

A. Heat map showing microRNAs significantly deregulated during aging in the "learning group". B. Fuzzy c-means clustering indicating the two main clusters of microRNAs differentially expressed in the learning group during aging. (C-D) Top biological processes and pathways identified based on experimentally validated target genes of the 55 microRNAs differentially expressed during aging.

\section{Integrative analysis of mouse and human data suggests a "microRNA signature" as a}

\section{biomarker for early onset of cognitive dysfunctions}

To identify microRNAs indicative for cognitive performance in our longitudinal study, we employed an unbiased microRNA feature selection approach using Random Forest-based machine learning. First, we subjected the different water maze features to a principle component (PCA) analysis to identify the key factors explaining most of the variability in the data. Most of the variability was explained by principal components 1 and 2 (PC1, PC2) (Fig S4). Next, to identify microRNAs informative about memory performance, we employed PC1 scores to subject the 55 aging-responsive blood microRNAs via two 
independent methods for feature selection (Table S5), namely multivariate bootstrapping and multivariate leave one out cross-validation (Looc; see method for more details). Both approaches identified three microRNAs linked to memory performance consisting of microRNA-181a-5p, miR -146a-5p and microRNA-148a-3p and were termed as "microRNA signature". microRNA members from "microRNA signature" were also part of the brown and blue co-expression modules linked to cognitive performance in healthy humans (Table S1, Fig 1), an observation that guided us to hypothesize that "microRNA signature" can potentially inform about cognitive status. Since these microRNAs are also part of "aging responsive" microRNAs (Table S4), their correlation with cognition could also be by chance, and therefore, they may not be informative.

To investigate this in further detail, we analyzed the expression of "microRNA signature" in several human and mouse datasets related to aging and cognitive dysfunction. To this end, we calculated the co-expression of all three microRNAs based on singular value decomposition on expression data. Since, we previously observed early cognitive deficits in mice between 13.5 and 16.5 months (see Fig 2, Fig 4A), we tested whether coexpression microRNAs could drive this cognitive transition. Of note, the cognitive deficit was highest at 16.5 months (see Fig 2, Fig 4A). We found increased co-expression of "microRNA signature" in the blood of mice at both 15 and 16.5 months of age compared with 13.5 months (Fig 4B). Increased co-expression of the signature at both 15 (no cognitive deficits observed) and 16.5 (substantial cognitive deficits observed) months hint toward this signature's potential as a marker for early cognitive decline. 


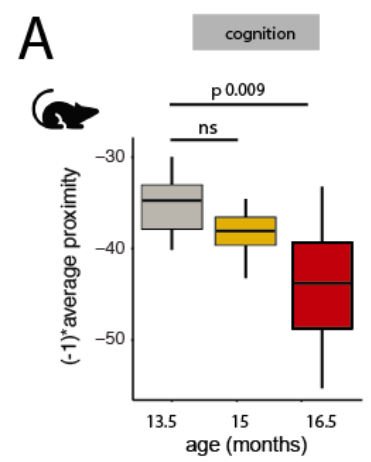

B
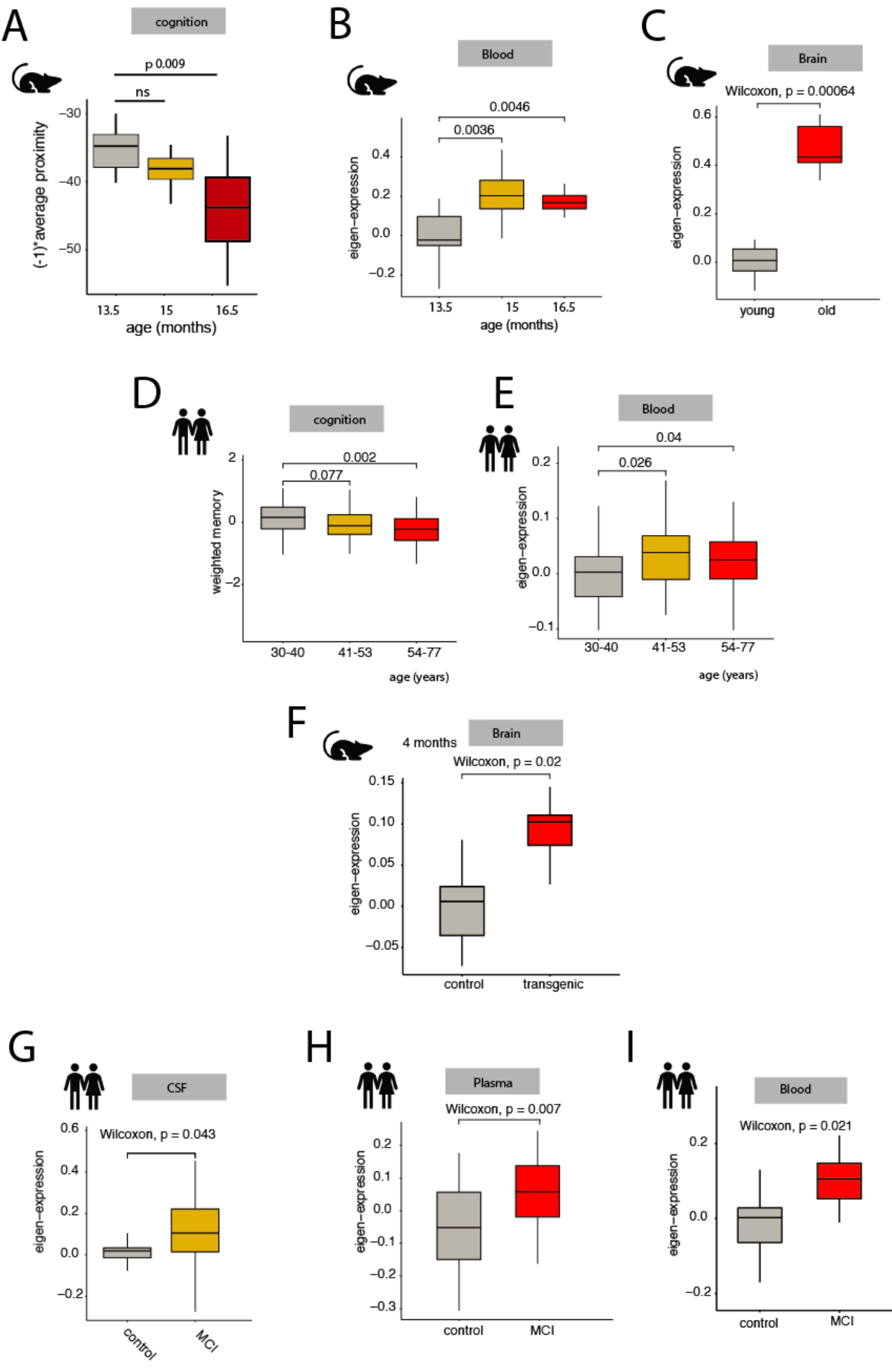

$\begin{array}{lr}\text { Fig 4. A } & \text { Multi-step } \\ \text { approach } & \text { finds } \\ & \text { "microRNA }\end{array}$

signature".

(A-I) Eigen-expression

of these three

microRNAs were used

to assay their co-

expression in several datasets. Before eigenexpression calculation, age, sex, batch, and surrogate variables (determined by sva bioconductor package) were taken into consideration as per the data availability of the given experiment and fit into a mixed linear model to adjust expression data for these effects. A. Mice show cognitive impairment at 16.5 months as represented by decreased (1)*average proximity during water maze test compared to 13.5 months old mice (Wilcoxon test, $* * \mathrm{p}<0.01, \mathrm{n}=10$ each group). Of note, increased average proximity indicates poor performance. For visualization, inverse signed average proximity has been plotted B. Coexpression of conserved three "microRNA signature" are longitudinally increased along with aging in mouse blood from 13,15 and 16 months (Wilcoxon test, ${ }^{*} \mathrm{p}<0.05, \mathrm{n}=10$ each group). C. Eigen-expression is increased in the brain of young $(n=8)$ compared to old $(n=9)$ mice (Wilcoxon-Rank test, $* \mathrm{p}<0.05)$ näive B6J mice. D. Healthy human subjects were classified into three cross-sectional age groups ("30-40”, “41-53" and "54-65" years of age group). Weighted cognitive score (see Methods for details about calculation) from corresponding cross-sectional age group shows a significant decline in cognitive performance at "54-65" $(\mathrm{n}=29)$ age group compared to "30-40" $(\mathrm{n}=36)$ age group (Wilcoxon test, $* \mathrm{p}<0.05)$. Similar to mice, there was a trend in cognitive decline in "41-53" $(\mathrm{n}=38)$ age group of human subjects compared to "30-40" year age group. E. "microRNA signature" eigen-expression from sex effect adjusted expression increased at " $41-53 "(\mathrm{n}=37)$ and " $54-65 "(\mathrm{n}=31)$ age groups compared to "30-40" year $(\mathrm{n}=41)$ age group (Wilcoxon test, $* * \mathrm{p}<0.01$, $*_{\mathrm{p}}<0.05$ ). F. In APPS1 mice brain, model for Alzheimer's disease, co-expression of the "microRNA signature" increased in transgenic mice compared to littermate controls at four months ( $\mathrm{n}=6$ each group) (Wilcoxon Rank test, $* \mathrm{P}<0.05, * * \mathrm{P}<0.01$ ). G) "microRNA signature" co-expression in CSF (control, $\mathrm{n}=16$; $\mathrm{MCI}, \mathrm{n}=12$ ) H. "microRNA signature" co- 
expression was also increased in plasma of human subjects who displayed mild cognitive impairment ( $\mathrm{n}=$ 6) compared to neurologically healthy subjects $(n=16)$. I. "microRNA signature" expression in blood (control, $n=23 ; \mathrm{MCI}, \mathrm{n}=52$ ). Age, gender, and effects from other surrogate variables as determined by sva were adjusted during the analysis of expression data (Wilcoxon Rank test). Box plot centers, lower and upper hinges represent the median, first and third quartiles respectively.

The observation that the "microRNA signature" were also linked to neuron-related processes (see Table S7) prompted us to test their role in the brain more specifically. To this end we performed small RNA sequencing of the hippocampal sub-regions CA1, CA3 and dentate gyrus (DG) and the anterior cingulate cortex (ACC) isolated from age-matched young and cognitively impaired old mice (Fig S5, Table S8, Table S9) followed by differential expression and comparative analysis across tissues as summarized in Fig S5. Similar to the data obtained in blood samples, expression of the "microRNA signature" was significantly increased in the brains of old mice (Fig 4C).

To further substantialize the previous observation that our signature's expression in blood changes prior to cognitive decline, we decided to investigate its expression in human crosssectional data. Thus, we recruited healthy human individuals aged between 30 and 65 years and collected blood samples and assessed how cognitive performance is affected by aging. Previous cross-sectional ${ }^{39}$ and longitudinal studies ${ }^{40}$ reported that few measures of cognitive performance required for everyday activities start to decline at the early $30 \mathrm{~s}$. While such a decline in a few cognitive domains is evident from 40 years, most of the them decline after 53 years of age, ${ }^{41,42}$. Therefore, we divided the human mid-life age (4065 years $)^{43}$ into two cross-sectional age groups " $40-53$ " and "54-65" years of age. Next, we contrasted cognitive performance from these groups with that from " $30-40$ " years age group. For cognitive measure, we investigated weighted cognitive performance in our PsyCourse cohort. We observed a significant decline in cognitive performance in only "54-65" age group ( $<$ 0.05) (Fig 4D). Similar findings were reported in other studies 40,41,44,45. Next, RNAs collected from individuals were subjected to small RNA sequencing. After adjusting gender bias, we observed increased co-expression of our "microRNAsignature" at both "40-53" (cognitive decline was not significant at $\mathrm{p}<0.05$ ) and "54-65" (observed significant cognitive deficits) years group compared to "30-40" years group (Fig 4E). Therefore, similar to mouse, in human, expression of this signature increased 
significantly prior to significant decline in cognitive performance, and it remains overexpressed during memory impairment. In summary, our data finds that "microRNA signature" expression increase in the blood of both mouse and human before the early onset of cognitive decline and its increased expression is maintained when cognitive deficits are detected.

Analyses so far tested how this "microRNA signature" cluster expression changes during age-related memory decline. Next, we asked whether we would observe similar changes in age-matched disease conditions. Therefore, we investigated the co-expression of the signature in both blood and brain from mouse and human brain diseases. To this end, first, we analyzed the expression of the "microRNA signature" in APPS1 mice, a model for amyloid deposition and $\mathrm{AD}$, at four months of age. Corroborating the previous findings, we observed increased expression of "microRNA signature" in the brain of four months old APP/PS1 mice compared with age-matched controls (Fig 4F). Of note, at four months of age mice, APP/PS1 mice display mild cognitive impairment (MCI) (Agbemenyah....Fischer A. et al., unpublished). Since the corresponding expression data from human MCI brain was not available, we investigated microRNA expression in MCI patient's cerebrospinal fluid (CSF) as a proxy to the brain. After removing age, gender, and other surrogate covariates, we found increased co-expression of "microRNA signature" in CSF from human individuals with MCI compared with that of neurologically healthy subjects (Fig 4G, Jain,...Fischer A. et al. 2019, in press). Moreover, increased expression of "microRNA signature" was also observed in human plasma from MCI patients compared to age-matched healthy controls (Fig 4H). Furthermore, increased coexpression of "microRNA signature" was observed in blood from MCI patients compared to healthy subjects (Fig 4I). In summary, our data suggest that our circulating "microRNA signature" can inform about cognitive status at an earlier stage of cognitive deficit in mouse and human and might help to diagnose individuals with early cognitive deficits. 


\section{Manipulation of microRNA-181a-5p, member of "microRNA signature", rescues early cognitive deficits in mice}

Having an early cognitive decline informative "microRNA signature", we asked whether we could rescue early cognitive deficits. We reasoned that members of "signature microRNAs" might be a good choice for therapeutic interventions. To this effect, we were driven by studies showing that early synaptic dysfunction and neuronal loss are critical features for early cognitive defects ${ }^{46,47}$. Thus, we sought therapeutic microRNA that is highly expressed in the brain and particularly enriched in neurons and could affect synaptic function. We first examined the neural cell-type expression of all three microRNAs from our "microRNA signature" 48 and observed that microRNA-181a-5p was highly enriched in neurons (Fig 5A) and has been previously linked to be active at the synapse and regulate cognate synaptic mRNA expression ${ }^{49}$. Therefore, we decided to characterize the role of microRNA-181a-5p in cognition.

Our qPCR data confirmed an increased expression of microRNA-181a-5p in both blood and brain of middle age compared to young subjects (Fig 5B). Functional analysis using target genes revealed that microRNA-181a-5p is likely to regulate pathways linked to cognitive aging such as insulin, GPCR and MAPK signaling as well the function of the glutamate synapse and long-term potentiation (Fig 5C). These result point towards the link between microRNA-181a expression and cognition. We asked whether changes in microRNA-181a-5p expression could be causative or compensatory for early cognitive deficits. To investigate this in great detail, we revisited the longitudinal cohort and observed that increased microRNA- 

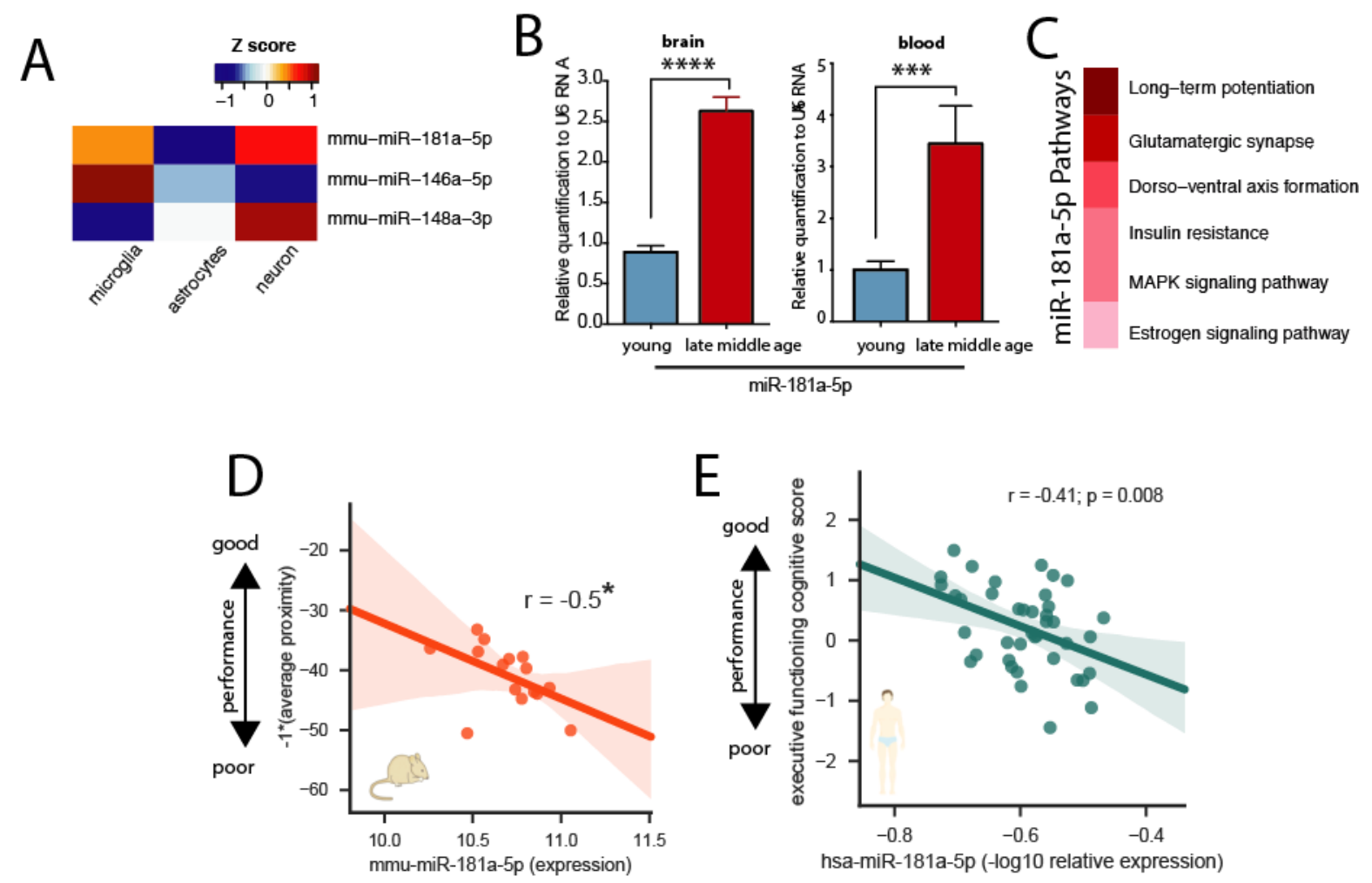

Fig 5: Circulating microRNA-181a levels, one of the microRNAs from our "microRNA signature", is linked to memory performance in mice and humans

A. Heat map showing the expression of these three microRNAs in different neural cell types based on the published dataset B. Bar plot showing qPCR results confirming the up-regulation of microRNA-181a-5p expression in brain $(n=6$ each group, two-tailed t-test, $\mathrm{p}<0.05)$ and blood $(\mathrm{n}=8$ each group, two-tailed t-test, $\mathrm{p}<0.05$ ) of old age when compared to young animals. C. microRNA-181a-5p related biological pathways D. Negative correlation between average proximity and microRNA-181a-5p expression from 15-16.5 months (Pearson's correlation). A negative sign of average proximity has been used to make the plot. E. Correlation of microRNA-181a-5p levels in blood assayed via qPCR in 40 randomly selected individuals out of the PsyCourse cohort with cognitive score shows a negative correlation of microRNA-181a-5p with cognition $(\mathrm{r}=-0.41, P=$ 0.006). Error bars indicate mean \pm standard error mean.

181a-5p expression levels in the blood is negatively correlated with cognitive performance (Fig 5D). Of note, at 16.5 months, mice exhibit increased inter-individual variability (Fig S6A), a finding that is also true for cognitive performance in humans ${ }^{50}$. Indeed, the link between microRNA-181a-5p and interindividual variability in healthy humans was confirmed (cor $-0.41, * * * p 0.006$ ) via qPCR of 40 randomly selected individuals from our cohort (Fig 5E). Collectively, these data suggest that there is a negative association between increased microRNA-181a-5p expression and early cognitive deficit. Therefore, we hypothesized that increased microRNA-181a-5p expression could be causative for an early cognitive deficit. 
A

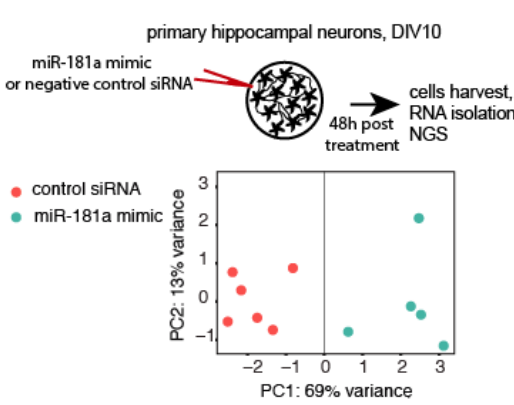

D

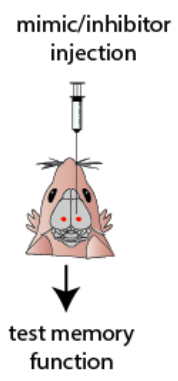

B
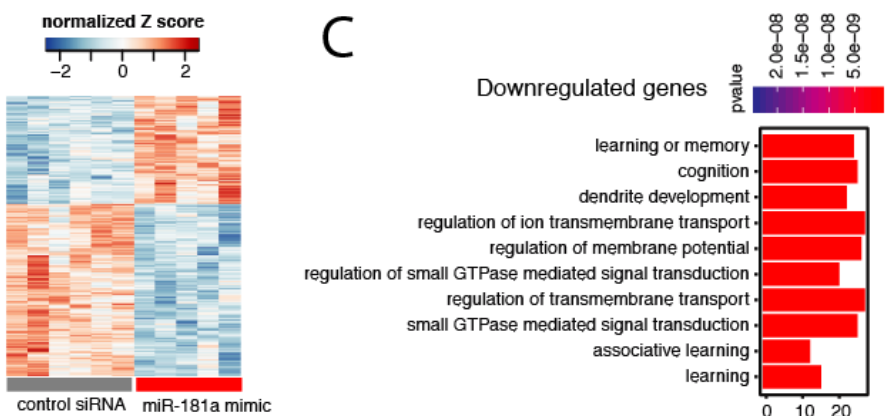

$\mathrm{E}$
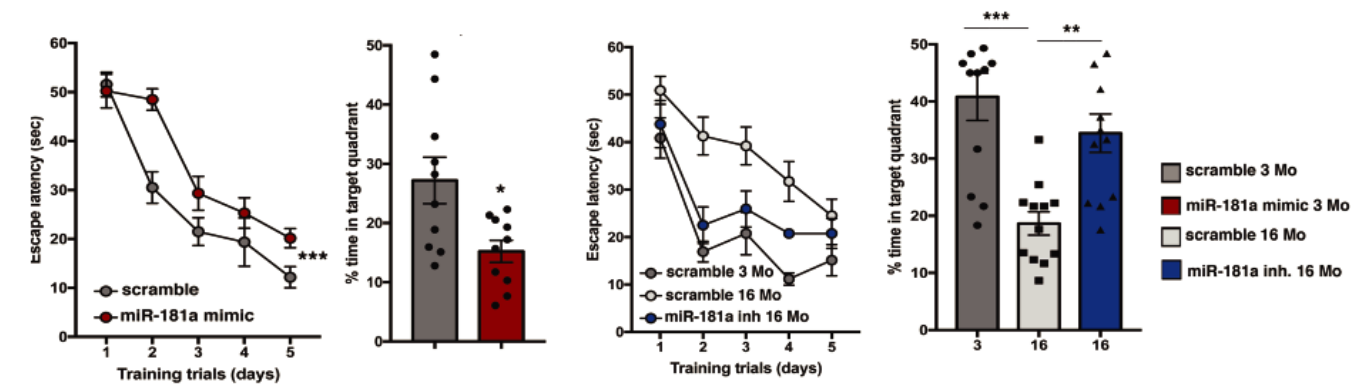

Fig 6: microRNA mediated rescue of early cognitive deficit

A. Primary hippocampal neurons (DIV10) were transfected with ncSiRNA/microRNA-181a-5p for $48 \mathrm{~h}$ prior to the collection of cells followed by RNA extraction and next-generation sequencing. PCA plot of the sequenced data showing the biological difference ( $69 \%$ variance along with first principle component) between ncSiRNA and microRNA-181a-5p mimic treated cells $(\mathrm{n}=6$, each group). B. Heat map showing differentially expressed genes (adjusted $\mathrm{p}$-value $<0.05$ ) between two groups. Two hundred twelve genes were up-regulated, while 335 genes were down-regulated in microRNA-181a mimic treated neurons compared to control groups. Down-regulated genes are termed as "DownRegulatedCluster". C. Gene ontology of down-regulated genes. Top 10 significant (adjusted p-value $<0.05$ ) biological processes are displayed. Learning, memory, and cognition related processes were top hits, suggesting that "DownRegulatedCluster" genes may be involved in cognition related pathways. D. Left panel: Experimental design. microRNA-181a-5p mimic or inhibitor oligonucleotides were injected into the CA1 of the dorsal hippocampus via microcannula prior to behavior testing. Middle panel: Escape latency is significantly reduced in mice injected with microRNA-181a-5p mimic when compared to the corresponding scramble control group ( ${ }^{* * *} \mathrm{p}<0.001, \mathrm{~F}=41.4$ for treatment; 2 way ANOVA). Right panel: During the probe test mice injected with microRNA-181a-5p mimic display reduced time in the target quadrant when compared to the scramble control group $\left({ }^{*} p=0.012\right)(n=10 /$ group $)$. E. Left panel: The escape latency of 16 months old mice injected with control oligonucleotides is reduced when compared to corresponding 3-months old mice ( $\mathrm{p}<0.0001, \mathrm{~F}=17.1,2$ way ANOVA) or to 16 months old mice treated with microRNA-181a-5p inhibitor ( $p<0.0001, \mathrm{~F}=14.8,2$ way ANOVA) Right panel: 1way ANOVA revealed a significant group difference in the time spent in the target quadrant during the probe text $(\mathrm{p}<0.001, \mathrm{~F}=11.96)$ and post hoc analysis revealed that 16 months old mice injected with scramble control oligonucleotides were impaired when compared to corresponding 3 -months old mice $(* * * p<0.0001)$ or 16 months old mice injected with microRNA-181a-5p inhibitors $(* * \mathrm{p}=0.006)(\mathrm{n}=10$ /group $)$. Error bars indicate mean $\pm \mathrm{sem}$.

To test this hypothesis to further detail, we decided to examine the role of microRNA$181 \mathrm{a}-5 \mathrm{p}$ on neuronal function and memory directly. To this end, we overexpressed microRNA-181a mimic in hippocampal neurons and performed RNA sequencing. PCA revealed distinct expression patterns between control and mimic treated neuronal cells (Fig 
6A). Further analysis revealed many significantly differentially expressed genes (Table S11) and as expected, the majority of the genes were down-regulated (335) (Fig 6B). 11\% of these genes overlapped with confirmed targets of microRNA-181a deposited in miRTarBase. These target genes include histone methyltransferases Kmt2a and Kmt2c (Table S12). GO-term analysis of the down-regulated genes revealed pathways linked to learning and memory (Fig 6C) further supporting the role of microRNA-181a in cognitive dysfunction. Interestingly, the genes down-regulated in response to elevated microRNA181a levels were highly overlapped with that from mouse models for cognitive disorders and human neurodegenerative diseases (Fig S8). These results imply a conserved microRNA-181a regulated network may underpin cognitive deficit in dementia. Altogether, these data provide evidence to claim that microRNA-181a is likely to cause cognitive deficits.

To test this claim directly, we implanted microcannula into the CA1 region of the dorsal hippocampus of 3 months old mice and injected microRNA-181a-5p mimic oligonucleotides using an established protocol $^{23}$ (see Fig 6D). Mice injected with a scrambled control oligonucleotide were used as control group. We used short oligonucleotides as control (scramble control) that would not interfere with endogenous transcript level for any gene. When subjected to water maze learning, mice injected with microRNA-181a-5p showed impairment in both learning and memory compared to control group (Fig 6D). Similar data were obtained using another memory test that measures associative contextual fear memories (Fig S9). These findings indicate that increased hippocampal microRNA-181a-5p levels contribute to memory impairment and suggest that in turn, reducing microRNA-181a-5p levels in the brain of aged mice could be a novel therapeutic approach to improve cognition. To test this hypothesis, we implanted microcannula into the dorsal hippocampus of 16 months old mice, the time point when we observed early cognitive deficit, and subsequently injected a microRNA-181a-5p inhibitor oligonucleotide. We had two control groups from 3 and 16-months old mice that were injected with a scrambled control oligonucleotide. Mice were then subjected to the water maze and fear-conditioning test to examine spatial reference and associative fear memories. While performance in the water maze was impaired when comparing scramble injected 16 to 3 months old mice, this effect was ameliorated in 16 months old mice 
injected with the (Fig 6E). Similar data were observed in the fear-conditioning paradigm (Fig S9).

In conclusion, we identify a "microRNA signature" that can inform about the early onset of cognitive deficits in both humans and mice. Moreover, our study demonstrates that early cognitive deficits in mice could be ameliorated through manipulation of one of the members of "microRNA signature", namely, microRNA-181a-5p. We suggest that the analysis of the identified "microRNA signature" will be informative in ongoing and future prospective clinical and epidemiological studies investigating the cognitive function and brain plasticity in humans. Finally, our experimental approach is suitable for the identification of circulating biomarkers in other human diseases. Therefore, we encourage researchers to follow the experimental strategy outlined in our study by combining research on model system and humans, which will help to build an unprecedented database for the analysis of blood small RNAs as biomarker for human diseases. 


\section{Discussion}

There is increasing interest in the analysis of the blood microRNAome as biomarker for cognitive disease (e.g., see $\left.\begin{array}{llll}13 & 14-16 & 17 & 18\end{array}\right)$. Our primary aim was to identify circulating microRNAs as non-invasive biomarkers that could diagnose early cognitive changes in dementia. We decided first to address this issue in humans. We reasoned that the crosssectional comparison between healthy and demented individuals would be suboptimal to discover informative biomarkers for early cognitive decline. Therefore, we adapted an alternative approach where we investigated total blood microRNAs in healthy human subjects who displayed asymptomatic cognitive variability in various neuropsychiatric tests. Our analysis revealed that microRNAs that significantly correlate with composite cognitive variabilities in healthy humans are likely to be involved in hypoxia. We have recently shown that hypoxia could drive the deregulation of cognition related changes in the brain and thereby can contribute to cognitive deficits (Md Rezaul Islam et al. 2019 unpublished). Changes in microRNAs implicated in hypoxia may reflect the early metabolic changes occurring in the incipient demented brain. Concordantly, other studies have reported that hypoxia can be a risk factor of dementia ${ }^{51,52}$. Additionally, correlated microRNAs are also likely to be involved in aging, another risk factor of dementia. Given that the human participants in this study are young (age $25.95 \pm 5.1$ years), observing changes in aging-related microRNA expression and its association with cognition in this cohort is intriguing and prompted us to investigate further the effect of aging on microRNAs.

To this end, we investigated blood microRNA expression and cognition in mice longitudinally. To analyze cognitive performance, we employed the Morris water maze test, a sensitive method to measure age-associated memory impairment in mice ${ }^{36}$ and humans ${ }^{33}$, that allows a repeated analysis of the same individual at multiple time points 34,53. By positioning platform at different locations, we examined changes in cognitive performance over an extended time in mice. Memory impairment became most evident between 15 and 16.5 months of age. These data are in line with previous findings from cross-sectional studies, where memory impairment in mice was detected at 16 months ${ }^{36,54-}$ 56. Our data from mice are also in line with findings in humans. Approaches to compare the relative age between different species suggest that mice of 15-16.5 months of age 
represent 50-60 years age range in humans, a time window when memory decline sharply in the latter ${ }^{50} 57$. In agreement with this, we observed a cognitive decline in humans aged 54 and above (Fig 4D). Next, via a number of complementary computational approaches, we identified a "microRNA signature" in blood composed of three microRNAs (microRNA-181a-5p, microRNA-146a-5p, microRNA-148a-3p) that show increased coexpression before and during early cognitive decline in both healthy mice and humans. Interestingly, the increased co-expression of "signature microRNAs" in blood was also observed during mild cognitive impairment (MCI) in patients with Alzheimer's disease (AD). A similar pattern in expression was further observed in the $\mathrm{AD}$ brain, at least in mice. Thus, the distinct co-expression pattern of circulating "microRNA signature" could reflect changes occurring in the brain and more importantly could inform about early cognitive deficits.

The question, however, remains why co-expression of these microRNAs levels similarly regulated in blood and the brain. To this end, it is interesting to note that microRNA-146a$5 p$, microRNA-148a-3p, and microRNA-181a-5p are highly expressed in blood and brain $^{58}$. All three microRNAs that belong to "microRNA signature", have been shown to be expressed and playing critical roles in B-lymphocytes ${ }^{59-61}$. B-lymphocytes are known as "memory cells" that are characterized by the formation of an immune-synapse which shares structural similarity to the neuronal synapse and is critical for the regulation of $\mathrm{T}$ and B-cell function ${ }^{62}{ }^{63}$. It is therefore tempting to speculate that the co-expression of "microRNA signature" in neural cells and lymphocytes could be controlled via similar mechanisms that result in the early memory decline. Moreover, changes in the adaptive immune system have been linked to memory decline and neurodegeneration ${ }^{64}{ }^{65}$. Future research will address the role of the immune system on modulating the expression of the "microRNA signature". However, the first evidence for this hypothesis stems from the fact that the promoter of microRNA-181a harbors, for example, binding motifs for NF- $\mathrm{BB}$ (Table S13). Moreover, previous studies have shown that microRNA-146a-5p expression can be regulated by $\mathrm{NF}-\mathrm{KB}^{60,66}$. Of note, this transcription factor has been linked to adaptive immunity and immune synapse function ${ }^{67,68}$ as well as memory formation 6937 ${ }^{70}$ and Alzheimer's disease ${ }^{71}$. Another possible scenario is that the observed changes in "microRNA signature" co-expression reflect age-related changes in lymphocyte numbers. This can, however, not explain the increased co-expression of "microRNA signature" in 
age-matched individuals and moreover, lymphocyte number is known to generally decrease with increasing age ${ }^{72}$. Thus, a simple overall change in blood cell number cannot explain the observed increase in circulating "microRNA signature" co-expression. It is also possible that circulating levels of "microRNA signature" represent an increased coexpression pattern in other organs. For example, microRNAs can be released from cells via exosomes ${ }^{73}$, and brain-derived exosomes have been identified in circulation ${ }^{74}$. Indeed, all the members of the "microRNA signature" are present in CSF exosomes (unpublished data) and we observed increased co-expression of the "microRNA signature" in this biological fluid (Fig 4G). Moreover, increased expression of "microRNA signature" was also observed in the plasma of MCI patients, highlighting a consistent expression pattern of the "microRNA signature" in various biological fluids. The method applied in this study to isolate RNA from blood, however, does not allow to identify the precise source of the detected microRNAs. Thus, further studies are required to elucidate the exact mechanisms that lead to increased co-expression of "microRNA signature" levels in both blood and brain.

As an exemplar candidate from this cluster of three microRNAs, we further studied microRNA-181a-5p mechanistically. Conclusive evidence that microRNA-181a-5p is causative to cognitive decline was provided by our data showing that increasing microRNA-181a-5p levels in the dorsal hippocampus of young mice impaired both spatial reference and associative learning. By contrast, two recent studies have reported that miR$181 \mathrm{a}-5 \mathrm{p}$ is important for memory formation ${ }^{75,76}$. However, in support of our observation, elevated levels of microRNA-181a-5p were implicated in cell death after cerebral ischemia, whereas low levels were associated with neuronal survival 7778 79. These supporting data are also in line with our observation that microRNA-181a-5p levels in the brain are highest in neurons and that pathways linked to experimentally validated microRNA-181a-5p targets represent key processes linked to synaptic plasticity and neuronal integrity. Indeed, microRNA-181a-5p was found in synapses, where it controls surface levels of key mRNAs linked to memory function such as AMPA-type glutamate receptor ${ }^{80}$ or CamKII ${ }^{81}$. As shown in this study, the potential of microRNA-181a-5p in regulating a conserved gene network in general memory disorders could explain, at least in part, how increased expression of microRNA-181a-5p can contribute to general cognitive deficits. It is thus interesting to note that microRNA-181a-5p levels in PBMCs 
are correlated with an increased genetic risk for autistic phenotypes in schizophrenia patients that are characterized by impaired cognition ${ }^{82}$. Interestingly, by decreasing levels of microRNA-181a-5p levels in the hippocampus of aged mice improved memory function. These findings are in agreement with previous data showing that calorie restriction led to a down-regulation of microRNA-181a-5p, which was linked to improved neuronal integrity ${ }^{83}$. These data are of particular importance since calorie restriction ameliorates age-associated memory impairment ${ }^{84,85}$.

In conclusion, our study suggests that a cluster of three circulating "microRNA signature" could be a suitable marker of early cognitive changes in dementia. Moreover, it highlights the potential of microRNA-181a inhibitor as therapeutic intervention in early dementia. To the best of our knowledge, a longitudinal cognitive phenotyping together with analyses of blood microRNAome has not been conducted so far. Our experimental approach can be adapted to other longitudinal studies in mice in the context of disease progression and therapeutic intervention. In fact, on the basis of the data presented here we have initiated the consequent analysis of circulating microRNAs within the cohort studies of the German Center for Neurodegenerative Diseases (www.dzne.de), the PsyCourse cohort (http://www.psycourse.de/index-en.html) and the corresponding animal models with the aim to construct a database that will allow unprecedented comparison and crosscorrelation of data from humans and corresponding animal models. Considering that the current therapeutic arsenal to treat, for example, $\mathrm{AD}$ might be effective if treatment can be initiated early, circulating "microRNA signature" biomarker could help to improve the treatment of dementia significantly. 


\section{Materials and Methods}

\section{Animals}

Naive male C57B/6J mice were obtained from Janvier. All animals were housed in standard cages on $12 \mathrm{~h} / 12 \mathrm{~h}$ light/dark cycle with food and water ad libitum. All experiments were performed according to the protocols approved by FELASA. Mice of 3 months and 18 months were sacrificed by cervical dislocation and whole brain was isolated in ice-cold Dulbecco's Phosphate Buffered Salt (DPBS, PAN-biotech GmbH) supplemented with EDTA-free protease inhibitor cocktail (Roche). The ACC, CA1, CA3 and DG regions were isolated, snap frozen in liquid nitrogen and stored at $-80{ }^{\circ} \mathrm{C}$. To follow the aging process in individual mouse, animals at age of 12 months were divided into two groups: normal aging (A) and water-maze aging (WM). Mice performing water maze related behavioral experiments belonged to water maze group and the rest were in control group. For water maze group, we started longitudinal experiment with 12 mice, but during analysis two mice were removed as outliers to have same 10 mice throughout different time points. All mice at 12 months age (before separating into two groups and starting behavioral tests) were considered as control for comparison of small RNA expression profiles at other age. APP/PS1-21 mice were from $\operatorname{Tg}$ (Thy1-APPSw,Thy1PSEN1*L166P)21Jckr colony.

\section{Human subjects}

All experiments reported in this study were approved by the local ethics committee. We analyzed healthy individuals that participated in PsyCourse study (Budde et al., in press; https://www.preprints.org/manuscript/201710.0169/v1). Briefly, adult healthy individuals were recruited for this study as control participants. The following neuropsychological tests were performed: Digit-Span (forward and backward); Digit-Symbol-Test, TrailMaking-Test, and the Multiple-Choice Vocabulary Intelligence Test (MWT-B) for details see Budde et al. (in press). Samples were excluded from the study if they had ever been detected as patient for mental and behavior disorder of ICD-10 diagnoses. The study was approved by the local ethics committee that was in accordance with the declaration of Helsinki. A composite score considering all the psychological tests was calculated similar to ${ }^{86}$. Briefly, for exploratory factor analysis on cognitive test scores, number of optimum latent factors $(\mathrm{n}=3)$ was determined based on model fit score RMSEA 0.02 and parallel analysis ${ }^{87}$. Next, exploratory factor analysis was performed and tests were grouped into 
three cognitive domains. Based on the low loadings $(<0.4)$ on the factors, multiple choice vocabulary intelligence and trail making test error-A tests were discarded from the analysis. First domain was measured by Trail making tests and Digit Symbol test. Similar factor analysis based domain was reported and termed as executive function domain in previous study ${ }^{88}$. Second domain comprised of Digits forward, Digits backward and could be described as "working memory" domain. Errors in Trail making tests (part B) were part of the third cognitive domain. Having the cognitive domains defined, individual test score was standardized by subtracting the mean from the score and dividing by the standard deviation. Next, an average domain specific score was calculated from the standardized scores of the tests belonging to the domain. Finally, domain specific cumulative scores were averaged to obtain a global weighted cognitive performance score. Higher and lower weighted score represent good and poor performance respectively.

\section{Water maze}

A standard water maze setting was used to conduct Morris-water maze experiment as previous study ${ }^{35}$. At 12 months, first water maze training was performed to habituate mice with the training environment. Animals were tested for visual performance by putting a colored cue on the platform. In order to do the spatial memory-learning test and to check cognitive flexibility, during the later training sessions at 13.5, 15 months and 16.5 months platform was put at the center of a new quadrant. However, the position of platform was kept unchanged throughout the corresponding training session. Different visual cues on four sites of the pool were not changed during training trials either.

The swimming behavior of the mice was recorded by a camera set on top of the water pool and was analyzed by VideoMot2 (TSE). At each training session, mice were trained to swim to the hidden platform in four daily trials, starting from pseudorandomly varied locations. Each trial consisted of 60 seconds. If mouse failed to find the platform in due time it was gently guided to it. Once reaching the platform, the mouse was allowed to have rest for 15 seconds. After four consecutive trials per day, each mouse was returned to its home cage where it rested until the next day of training. Sixty seconds long probe trial was performed without the platform after each training session at a given month. After last probe test performed at 16.5 months, animals were tested for visual acuity again. 
For data analysis, features from TSE VideoMot2 software were used. For occupancy plot, 50 x 50 grids defined a bin and number of mouse crossing through each grid was summed up. Densities were calculated from the ratio of total number of crossings through each grid and total number of crossings in the bin. For plotting, a smoothened technique was applied to the densities and minimum and maximum densities out of all experimental groups were taken into consideration during normalization step.

For in depth feature analysis from water maze data, a modified version of MUST-C algorithm was used ${ }^{32}$. In addition to the features described in original paper, we extracted few extra features from raw water maze data including total coverage, distance from centroid to platform and path efficiency. The definition of the features extracted from water maze data are as follows:

a) Average proximity to platform: mean of Euclidean distances from all positions to the platform b) Distance from centroid to platform: Euclidean distance between center of all positions and platform c) Sum of absolute angles: sum of angles between pairs of sequential vectors d) Mean distance from perimeter: mean distance to the closest point on the pool's perimeter e) Total duration: total time mice spent in searching the platform f) Number of quadrant changes: total number of quadrant changes during trial g) Maximal time at one quadrant: percentage of time spent in a single quadrant h) Sum of relative angles: positive and negative angles are marked from positive and negative movements along $\mathrm{X}$ axis respectively i) Cumulative angle along $\mathrm{Y}$ axis: similar to angles along $\mathrm{X}$ axis, relative angles along $\mathrm{Y}$ axis is calculated j) Platform crossings: number of crossings of the platform region (probe test) k)Variance of distance from perimeter: variance of Euclidean distances from the pool's perimeter 1) mean velocity: ratio between total distance and total time spent m)Distance traveled: sum of Euclidean distance between each pair of sequential locations n) Total coverage: ratio between area of convex hull of all points and the area of the pool o) Local densities: mean distance between all pairs (x,y coordinates) of locations in the trial p) Variance of distance to platform: variance of Euclidean distance from all positions to the platform q) Path efficiency: ratio of the path length to the Euclidean distance between starting and endpoints. Path length is sum of Euclidean distances between all consecutive points in the trial.

Based on all these features scatter plots were generated, and one of the following search strategies was assigned to each trial of the training session. Different scores were assigned to these different strategies ranging from 0 to 10 . Briefly, direct, corrected, focused and 
short chaining represent higher cognition and therefore were given score of 10,9,9 and 8 respectively. Other strategies were given scores as follows: circling $=5$, accidental circling $=4$ long chaining $=2$, focused false $=1$, random $=0$, thigmotaxis $=0$ and passivity $=0$. Cumulative scores during training and probe test were calculated from sum of all strategic scores across training trials and during probe test respectively. Since during probe trial there was no platform in water maze, only focused, focused circling, circling and random strategies could be defined.

\section{Fear conditioning test}

Fear conditioning test was performed using TSE fear conditioning system. Mice were allowed to explore the context for $3 \mathrm{~min}$ prior to experiencing mild electric foot shock (constant current, $0.5 \mathrm{~mA}$ ) for $2 \mathrm{~s}$. After 24 hours, they were introduced to the same context for $3 \mathrm{~min}$ without receiving any foot-shock and associative memory was checked by inspecting their freezing behavior.

\section{Collection of Blood from mice and purification of total RNA from blood and brain sub-regions}

For APPS1 mice, peripheral blood was collected through cardiac puncture and stored in tubes provided by RNeasy Protect Animal Blood System (Qiagen, Hilden, Germany). For mice used in longitudinal study, blood was collected from retro orbital sinus of all mice for first time at 12 months age before starting any behavioral experiment. Next blood collections were done after 24 hours of finishing probe tests at 13.5, 15 and 16.5 months. In summary, individual mouse was anesthetized with Isofluran (1.8\%) for 2-3 minutes. Afterwards, 150-200 $\mu \mathrm{l}$ blood was collected from the orbital sinus of animals using a heparinized glass micro capillary and stored.in RNeasy Protect Animal Blood System tubes. Each time blood was collected from alternative eyes. The blood tubes were allowed to stand at room temperature for 24 hours, and then stored at $-20^{\circ} \mathrm{C}$. Purification of total RNA from blood was performed following the manual of RNeasy Animal Blood protect kit (Qiagen, Hilden, Germany). Briefly, blood tubes were centrifuged for 3 min at 5000xg at room temperature. After removing the supernatant without disturbing pellet, $1 \mathrm{ml}$ of RNAse-free water was added. The solution was vortexed and centrifuged in the same condition as mentioned above. Supernatant was discarded and $240 \mu 1$ Buffer RSB was added to pellets. After re-suspending the pellet properly, appropriate amount of buffer RBT and Proteinase $\mathrm{K}$ were added and the solution was incubated for 10 minutes at $55^{\circ} \mathrm{C}$. 
Next, the solution was applied on the membrane of the QIAshredder spin column and centrifuged for 3 minutes at the maximum speed. After centrifugation 1.5 volume of $100 \%$ Ethanol was added to the flow-through. Later, the solution was applied into a $2 \mathrm{ml}$ RNeasy MinElute Spin column and centrifuged for $1 \mathrm{~min}$ at 10,000xg. The membrane of spin column was washed once with $350 \mu$ l of Buffer RWT and DNase treatment was performed for $15 \mathrm{~min}$ at room temperature. Next, $350 \mu \mathrm{l}$ of buffer RWT was added to the column followed by centrifugation for 15 seconds at 10,000xg. Column was washed with $500 \mu 1$ Buffer RPE (centrifugation for 15 seconds, at 10,000xg, at room temperature). The final wash was performed with freshly prepared $80 \%$ ethanol followed by centrifugation for 2 minutes at 10,000xg. RNA was eluted with REB buffer after a short centrifugation $\left(10,000 \mathrm{xg}, 1\right.$ minute). Extracted RNA was incubated at $65^{\circ} \mathrm{C}$ for 5 minutes and immediately placed on ice. Concentration of RNA was measured on Nanodrop and isolated RNA was stored at $-80^{\circ} \mathrm{c}$ for future use. Total RNA extraction from mouse ACC, dentate gyrus, CA1 and CA3 were conducted using Tri reagent (Tri Reagent, SigmaAldrich,Germany) according to manufacturer's instruction.

\section{Blood collection in humans}

Blood was collected from the median cubital vein. Before collection the collection tubes were stored at $18-25^{\circ} \mathrm{C}$. For the collection of blood via PAXgene tubes $2.7 \mathrm{ml}$ of blood were filled into the tube that was subsequently gently inverted 10 times. Tubes were stored for $2 \mathrm{~h}$ at room temperature and then transferred to $-20^{\circ} \mathrm{C}$ for $24-72 \mathrm{~h}$ before being stored at $-80^{\circ} \mathrm{C}$ until further processing. For the isolation of PBMCs blood was collected in CPT tubes that were subsequently inverted 8 times and then centrifuged for $30 \mathrm{~min}(1700 \mathrm{xg})$ at room temperature. By using a pipette, plasma was removed (until $1 \mathrm{~cm}$ above the white lymphocyte layer). The white lymphocyte layer was then transferred into a $15 \mathrm{ml}$ Falcon-tube using a pipette. Subsequently the tube was filled with up to $15 \mathrm{ml}$ with PBS and gently inverted 5 times. The Falcon tube was centrifuged for $15 \min (300 \mathrm{xg}$, with brake) at room temperature and the supernatant was discarded and the pellet re-suspended in $15 \mathrm{ml}$ PBS. These steps were repeated in total 3 times. The cell pellet was eventually re-suspended in $2 \mathrm{ml}$ of PBS and frozen in liquid nitrogen before being stored at $-80^{\circ} \mathrm{C}$. For plasma samples, blood was collected into EDTAplasma tubes and centrifuged from $10 \mathrm{~min}(2000 \mathrm{xg}$, without brake) at room temperature. The supernatant was aliquoted into cryo-tubes into and stored within $30 \mathrm{~min}$ at $-80^{\circ} \mathrm{C}$. Exosomes were isolated from plasma via a number of subsequent centrifugation steps at $4^{\circ} \mathrm{C}: 3500 \mathrm{xg}$ for $10 \mathrm{~min}$, two times $4500 \mathrm{xg}$ for $10 \mathrm{~min}, 10000 \mathrm{xg}$ for $30 \mathrm{~min}$ and $100000 \mathrm{xg}$ for $60 \mathrm{~min}$. The 
100,000xg pellet was washed once with phosphate-buffered saline (PBS) at $100000 \mathrm{~g}$ for 60 min before re-suspension in $\mathrm{PBS}^{89}$

\section{High-throughput Small RNA sequencing and bioinformatics analysis}

Next generation sequencing of the RNA from collected blood samples from mouse were performed using TruSeq ${ }^{\circledR}$ Small RNA kit according to manufacturer's protocol (Illumina, San Diego, CA, USA). Briefly, 100 ng RNA was used as starting material followed by adapter ligation and primer hybridization. First strand of cDNA was generated followed by PCR enrichment. Libraries were pooled and PAGE was run for size selection. For small RNAome $\sim 150$ bp band was cut and used for library quantification after purification. A final library concentration of $2 \mathrm{nM}$ was used for sequencing. Sequencing was performed using a 50-bp single read setup on the Illumina HiSeq 2000 platform. Demultiplexing was done using Illumina CASAVA 1.8. For human samples, sequencing libraries were prepared using NEBNext ${ }^{\circledR}$ small RNA library preparation kit according to manufacturer's instruction.

Sequencing adapters were removed using cutadapt-1.8.1 ${ }^{90}$. Sequencing quality including total number of reads, percentage of GC content, sequence quality per base, $\mathrm{N}$ content per base, sequence length distribution, duplication levels, overrepresented sequences and Kmer content was investigated using FastQC v0.11.5 (http://www.bioinformatics.babraham. ac.uk/projects/fastqc/).

For quantification of mature microRNAs, miRDeep2 was primarily used. Briefly, mouse (mm10) and human (hg38) genome sequence were retrieved from UCSC (https://genome.ucsc.edu/) Genome Browser and corresponding genomic index files were created using Bowtie-build tool (version 1.12) with default options. Sequencing reads were mapped to the reference genome using mapper.pl script with default settings in the miRDeep2 package. Reads less than 18 nucleotides were discarded and miRDeep2 module with default options was used to identify known and novel microRNAs in sequencing data. To quantify known microRNAs, sequencing reads, the known mature microRNAs and optionally its star sequences for mouse were mapped against the known precursor microRNAs for reference genome documented in miRBase (Release 21), allowing 0 mismatch. A read was assumed to represent a sequenced mature microRNA, if it fell within the same position on the precursor as mature microRNA, plus $2 \mathrm{nt}$ upstream and $5 \mathrm{nt}$ 
downstream. For the prediction of novel microRNAs, mature microRNAs in Rattus norvegicus (miRBase release 21) were provided as related species for mouse. On the other hand, mature microRNAs in Gorilla gorilla, Pongo pygmaeus, Pan troglodytes (miRBase release 21) were provided as related species for human. However, novel microRNAs are not discussed in the current study. Samples were evaluated with respect to mapping quality and library size and only those with good mapping score and comparable library size were considered in downstream analysis. In total, for home-cage group we had sequencing data from 10 mice per time point while in water maze group sequencing data from 9, 10, 6 and 10 animals were analyzed for $12,13.5,15$ and 16 months, respectively.

\section{Differential expression analysis}

Prior to any differential analysis, microRNAs (from miRBase release 21) with raw read counts $\geq 5$ in at least $50 \%$ of studied samples were selected for further analysis. Unwanted variation factors were estimated based on reads in replicate samples and these variants were removed from sequencing data using RUVSeq (Risso, Dudoit et al. 2014). Differential expression analysis was performed using DESeq2 (Love, Huber et al. 2014). microRNAs having adjusted $\mathrm{p}$ value $<0.05$ were considered as significantly deregulated.

\section{Rank-rank hypergeometric overlap analysis}

The significance overlap of expressed microRNAs between two cohorts was calculated using the rank-rank hypergeometric test ${ }^{91}$. Briefly, microRNAs were ranked by expression, placing the most expressed microRNA at the top and the least expressed at the bottom of the list. Number of overlapping microRNAs between two cohorts was counted at every $10^{\text {th }}$ combination, and Fisher's exact test was used to calculate significance of the overlap and later was corrected after multiple statistical adjustments.

\section{Clustering of Expression Profiles}

For aging and WM groups, small RNAs were clustered according to their expression profiles using a fuzzy c-means algorithm in R based package e1071 v1.6-2. The optimum number of clusters was determined using several cluster validation indexes as described in Guthke et al. 2005. 


\section{Weighted gene co-expression analysis of microRNAs}

MicroRNAs that are highly correlated in learning group were identified and summarized with a modular eigengene profile using the weighted gene co-expression network analysis (WGCNA) package (version 1.61) in R. Briefly, normalized and log (base 2) transformed microRNA expression data was used to calculate pair-wise correlations between microRNAs. Next a soft threshold power of 8 was chosen based on approximate scale-free topology and used to calculate pair-wise topological overlap between microRNAs in order to construct a signed microRNA network. Modules of co-expressed microRNAs with a minimum module size of 20 was later identified using cutreeDynamic function with following parameters: method = "hybrid", deepSplit =3, pamRespectsDendro =T, pamStage $=\mathrm{T}$. Closely related module were merged using dissimilarity correlation threshold of 0.15. Different modules were summarized as network of modular eigengenes, (MEs) which were then correlated with weighted cognitive score calculated from eight different cognitive tests in human as mentioned above. Pearson correlation of MEs and each of these features was plotted as heat map and identified three modules (brown, blue and turquoise) that significantly $(\mathrm{p}<0.05)$ correlate with weighted cognitive performance.

\section{Machine learning based microRNA feature selection and prediction}

Normalized microRNA sequencing data was used to train a machine learning random forest regressor. A set of 17 features derived from the Morris Water Maze experiments were computed, namely average proximity to platform, centroid, cumulative angles, mean distance to edge, total duration, number of quadrant changes, maximum quadrant, relative angles, cumulative angle along y axis, platform crossings, pool edge variation, mean velocity, cumulative distance, total coverage, local densities, variation of distance to platform and path efficiency. An in-house data analysis toolbox was used to compute the aforementioned features. These phenotypic features were compressed into a single quantity by means of their first principal component projection (i.e. PCA's first component) and used as a univariate score for representing cognitive ability. The cognitive score was used along with the 55-microRNA count signatures to train a RF regressor, with the cognitive score being the outcome variable and the "microRNA signature", the predictors. Given our interest on determining the best (sub)-set of microRNAs that link to the cognitive score, three independent RF regression models were trained, each one using different feature selection algorithms: boostrapping, leave-one-out cross-validating and using a univariate filter. Parameters for bootstrapping were set to number $=250$, repeats $=1$ 
and $p=0.75$; whereas for the univariate filter, number $=5$ and repeats $=20$. Leave-one-out cross validation parameters were number $=1$, repeats $=1$ and $p=0.75$. Analyses were implemented using R (v.3.3.1) with the package Caret (v.6.0.76).

\section{Gene ontology and pathway analysis of microRNA target genes}

All experimentally validated microRNA target genes in mouse were retrieved from miRTarBase v-6.1 (http://mirtarbase.mbc.nctu.edu.tw/). Gene ontology and pathway analysis was performed using ClueGO v.2.2.6 ${ }^{92}$ platform in Cytoscape v.3.3 ${ }^{93}$. ClueGO functional analysis was performed using all experimental evidences (inferred from experiment, direct assay, physical interaction, mutant phenotype, genetic interaction and expression pattern) available. Enrichment of GO terms for set of microRNA cognate target genes was assessed with a right-sided hypergeometric statistical analysis which provides a p-value that was further corrected using a Bonferroni step down method. Only GO terms with corrected $p$-value $<0.05$ were considered as significant. Files were prepared using inhouse R script.

\section{Lipid nanoparticle packaging of microRNA}

Small RNAs (sRNAs) were packaged into lipid nanoparticles using SPARK using Neuro9 kit according to manufacturer's instruction. Briefly, re-constituted sRNA in nucleic acid storage buffer was mixed with formulation buffer 1 to have final concentration of sRNA of $930 \mathrm{ug} / \mathrm{mL}$. Next, formulation buffer 2, lipid nanoparticles and sRNA-formulation buffer were put in defined chamber of Spark Cartridge and a NanoAssemblr Spark was used to mix and generate lipid nanoparticles packaging sRNAs. All steps were followed in a sterile condition. Prepared lipid nanoparticles containing sRNAs were stored at $4^{\circ} \mathrm{c}$ before further use.

\section{Primary Hippocampal neuronal culture}

Whole hippocampus was micro-dissected from the E16.5 CD1 mice embryos and digested for 13 minutes at $37^{\circ} \mathrm{c}$ in $4.5 \mathrm{ml}$ pre-warmed PBS mixed with $2.5 \%$ trypsin. After digestion, dissociated cells were washed three times with processing media containing DMEM (Merck, cat. FG0445), 10\% FBS (GIBCO, cat. 10500064) and 1\% penicillin/streptomycin (PanReac AppliChem, cat. A8943). Cells were resuspended in the processing media and centrifuged at 300xg for 5 minutes to get cell pellet. Next, cell pellet was re-suspended in maintenance media containing neuroabasal, 1\% penicillin/streptomycin, 2\% B27 (GIBCO, cat. 17504001) and 1\% 
glutamax (Thermofisher Scientific, cat. 35050061) and plated in a 24 well PDL pre-coated plate at 130,0000 cells/well (DIV0). Neuronal enriched cultured was maintained at $37^{\circ} \mathrm{c}$ and on DIV10, cells were transfected with microRNA-181a-5p mimic/negative control siRNA. 48 hours post transfection; cells were washed, treated with Trizol, scraped and used for RNA isolation.

\section{Hippocampal injections}

Hippocampal injections to CA1 region were performed as described previously (Sananbenesi et al, 2007) ${ }^{23}$. In summary, mice were anaesthetized and microcannulae (gauges of the guide and injection cannula being 1.5 and $0.5 \mathrm{~mm}$ respectively) were inserted into the hippocampus using the following coordinates: $1.0 \mathrm{~mm}$ posterior to the bregma; $1.0 \mathrm{~mm}$ lateral and $1.5 \mathrm{~mm}$ ventral from midline. microRNA mimics and inhibitors were injected bilaterally ( $1 \mu \mathrm{l}$, at a rate of $0.3 \mu \mathrm{l} / \mathrm{min}$ per side).

\section{Quantitative RT-PCR for microRNA and target genes}

Quantification of mature microRNA-181a-5p in blood was accomplished using miScript Primer Assays kit (Qiagen, USA) on Roche LightCycler 480 instrument according to manufacturer`s instruction. For longitudinal samples in mice, mouse microRNA-29b-1 mature sequence was used as an internal control. For microRNA-181a-5p quantification from blood in young and old mice, human RNU6B was used as housekeeping gene. Real time PCR (q-PCR) primers for microRNA-181a-5p target genes MAP2k1, Insulin Growth Factor 1 Receptor (IGF1r) and AMPA2 (alpha 2), ionotropic glutamate receptor (Gria2) were designed using Universal probe library Assay Design Center and were purchased from Sigma. cDNA was synthesized using the Transcriptor High Fidelity cDNA Synthesis Kit (Roche) and real-time quantification was performed using Roche Universal Probe Library (UPL) probes. Primer sequences and UPL probe numbers for all the genes are given in Table S14. Results were normalized to the expression of internal control Hypoxanthine Phosphoribosyltransferase 1 (HPRT1) gene. Relative gene expression was analyzed by $2^{-\mathrm{ddCt}}$ method ${ }^{94}$.

\section{Statistics}

Statistical analysis for behavioral and molecular studies was performed using GraphPad Prism 7. Details of statistical analysis, methods, and parameters are given in the figure 
legends. Statistical tests performed were based on data structure and included as follows: one-way, two - way, or repeated - measures analysis of variance, Student's t-test and Ftest.

Acknowledgments: Authors thank Dr. Gaurav Jain for providing technical support. Moreover, Authors also thank Dr. Stefan Irniger, Dr. Rashi Halder, Dr. Johannes Söding, Dr. Saikat Banerjee and Dr. Torsten Klengel for their suggestions to the work. This work was supported by the following third-party funds to AF: DFG research group KFO241/PsyCourse Fi981-4 and Fi981 11-1, DFG project 179/1-1/2013, the ERC consolidator grant DEPICODE (648898), the BMBF projects ENERGI (01GQ1421A) and Intergrament (01ZX1314D), and funds from the German Center for Neurodegenerative Diseases FS was supported by the DFG grant SA1005/2-1and funds from the DZNE. PF is supported by the KFO241/PsyCourse project FA 241/16-1

Author contributions: MRI: participated in designing experiment, performed experiment, analyzed data, made figures and wrote the manuscript; LK: performed small RNA sequencing from psycourse healthy individuals and validated microRNA-181a-5p expression in human blood via qPCR TB: participated in designing experiment, performed experiment and reviewed the manuscript; TPC: performed feature selection based on sequencing and water maze data; VE: performed strategy analysis from water maze data; EB: Helped with interpretation of the data and reviewed the manuscript, PR: performed experiment; CK: shared basic scripts; MB: dissected mouse brains and prepared RNA; GJ: provided technical support; SB: provided sequencing facility; BM, HB, TS, PF, UH, MB collected and analyzed phenotypic data within the PsyCourse cohort, FS: designed the experiment; AF: designed the experiment, supervised and wrote the manuscript. 


\section{References}

$1 \quad$ Stern, Y. Cognitive reserve. Neuropsychologia. Neuropsychologia 47, 2015-2028 (2009).

2 Mangialasche, F., Solomon, A., Winblad, B., Mecocci, P. \& Kivipelto, M. Alzheimer's disease: clinical trials and drug development. Lancet Neurol 9, 702-716 (2010).

3 Bateman RJ, X. C., Benzinger TL, Fagan AM, Goate A, Fox NC, Marcus DS, Cairns NJ, Xie X, Blazey TM, Holtzman DM, Santacruz A, Buckles V, Oliver A, Moulder K, Aisen PS, Ghetti B, Klunk WE, McDade E, Martins RN, Masters CL, Mayeux R, Ringman JM, Rossor MN, Schofield PR, Sperling RA, Salloway S, Morris JC; Dominantly Inherited Alzheimer Network. Clinical and biomarker changes in dominantly inherited Alzheimer's disease. NEngl J Med. 367, 795-804 (2012). Schneider, L. S. et al. Clinical trials and late-stage drug development for Alzheimer's disease: an appraisal from 1984 to 2014. J Intern Med 275, 151-283 (2014).

5 Abbott, A. \& Dolgin, E. Leading Alzheimer's theory survives drug failure. Nature 540, 15-16 (2016).

6 Selkoe, D. J. \& Hardy, J. The amyloid hypothesis of Alzheimer's disease at 25 years. EMBO Mol Med 8, 595-608 (2016).

7 Weiner, M. W. et al. Alzheimer's Disease Neuroimaging Initiative. Impact of the Alzheimer's Disease Neuroimaging Initiative, 2004 to 2014. Alzheimers Dement., 7 (2015).

8 Witwer, K. W. Circulating MicroRNA Biomarker Studies: Pitfalls and Potential Solutions. Clin Chem. 61, 56-63 (2015).

9 Sheinerman, K. S., Tsivinsky, V. G., Abdullah, L., Crawford, F. \& Umansky, S. R. Plasma microRNA biomarkers for detection of mild cognitive impairment: biomarker validation study. Aging (Albany NY) 5, 925-938 (2013).

Rao, P., Benito, E. \& Fischer, A. MicroRNAs as biomarkers for CNS disease. Front Mol Neurosci. 26, eCollection 2013 (2013).

11 Kumar, S., Vijayan, M., Bhatti, J. S. \& Reddy, P. H. MicroRNAs as Peripheral Biomarkers in Aging and Age-Related Diseases. Prog Mol Biol Transl Sci. 146, 47-94 (2017).

12 Chen, R. J. et al. MicroRNAs as biomarkers of resilience or vulnerability to stress. Neuroscience $\mathbf{3 0 5}$ (2015).

13 Leidinger, P. et al. A blood based 12-miRNA signature of Alzheimer disease patients. Genome Biol. 14, R78 (2013).

14 Galimberti, D. et al. Circulating miRNAs as potential biomarkers in Alzheimer's disease. J Alzheimers Dis. 42, 1261-1267 (2014).

15 Cheng, L. et al. Prognostic serum miRNA biomarkers associated with Alzheimer's disease shows concordance with neuropsychological and neuroimaging assessment. . Mol Psychiatry 20, 1188-1196 (2015).

16 Schwarz EC, B. C., Knörck A, Ludwig N, Leidinger P, Hoxha C, Schwär G,, Grossmann T, M. S., Hart M, Haas J, Galata V, Müller I, Fehlmann T, Eichler \& H, F. A., Meder B, Meese E, Hoth M, Keller A. Deep characterization of blood cell miRNomes by NGS. Cell Mol Life Sci. 73, 3169-3181 (2016). Hara, N. et al. Serum microRNA miR-501-3p as a potential biomarker related to the progression of Alzheimer's disease. . Acta Neuropathol Commun. 2017 5, doi: 10.1186/s40478-40017-40414-z (2017).

18 Nagaraj, S. et al. Profile of 6 microRNA in blood plasma distinguish early stage Alzheimer's disease patients from non-demented subjects. Oncotarget 8, 16122-16143 (2017).

19 Gurtan, A. M. \& Sharp, P. A. The Role of miRNAs in Regulating Gene Expression Networks. J Mol Biol pii, Epub ahead of print (2013).

20 Lin, Q. et al. The brain-specific microRNA miR-128b regulates the formation of fear-extinction memory. Nat Neurosci 14, 1115-1117 (2011).

21 Gao, J. et al. A novel pathway regulates memory and plasticity via SIRT1 and miR-134. Nature 466, 1105-1109 (2010).

22 Hébert, S. S. et al. Loss of microRNA cluster miR-29a/b-1 in sporadic Alzheimer's disease correlates with increased BACE1/beta-secretase expression. Proc Natl Acad Sci U S A. 205, 6415-6420 (2008). Zovoilis, A. et al. microRNA-34c is a novel target to treat dementias. The EMBO journal 30, 4299-4308 (2011).

24 Aksoy-Aksel A, Z. F., Schratt G. MicroRNAs and synaptic plasticity--a mutual relationship. Philos Trans R Soc Lond B Biol Sci. 369, pii: 20130515 (2014).

25 Zovoilis, A. et al. microRNA-34c is a novel target to treat dementias. EMBO J 30, 4299-4308 (2011).

26 Banzhaf-Strathmann, J. et al. MicroRNA-125b induces tau hyperphosphorylation and cognitive deficits in Alzheimer's disease. EMBO J 33, 1667-1680 (2014).

27 Beason-Held, L. L. et al. Changes in brain function occur years before the onset of cognitive impairment. Journal of Neuroscience 33, 18008-18014 (2013). 
Sperling, R., Mormino, E. \& Johnson, K. The evolution of preclinical Alzheimer's disease: implications for prevention trials. Neuron 84, 608-622 (2014).

29 Deary, I. J. et al. Age-associated cognitive decline. British medical bulletin 92, 135-152 (2009).

30 Ludwig, N. et al. Distribution of miRNA expression acrosshuman tissues. Nucleic Acids Res. 44, $3865-$ 3877 (2016).

31 Duzel, E., van Praag, H. \& Sendtner, M. Can physical exercise in old age improvememory and hippocampal function? Brain 139, 662-673 (2016).

32 Illouz, T., Madar, R., Louzon, Y., Griffioen, K. J. \& Okun, E. Unraveling cognitive traits using the Morris water maze unbiased strategy classification (MUST-C) algorithm. . Brain Behav Immun. 52, 132-144 (2016).

33 Laczó, J. et al. Exploring the contribution of spatial navigation to cognitive functioning in older adults. Neurobiol Aging 51, 67-70 (2017).

34 Havas, D. et al. A longitudinal study of behavioral deficits in an A $\beta$ PP transgenic mouse model of Alzheimer's disease. J Alzheimers Dis. 25, 231-243 (2011).

35 Benito, E. et al. HDAC inhibitor-dependent transcriptome and memory reinstatement in cognitive decline models. The Journal of clinical investigation 125, 3572-3584 (2015).

Peleg, S. et al. Altered histone acetylation is associated with age-dependent memory impairment in mice. Science 328, 753-756 (2010).

37 Stilling, R. M. et al. De-regulation of gene expression and alternative splicing affects distinct cellular pathways in the aging hippocampus. Front Cell Neurosci. 8, doi: 10.3389/fncel.2014.00373. (2014). Flurkey, K., Currer, J. M. \& Harrison, D. E. in The mouse in biomedical research 637-672 (Elsevier, 2007).

39 Park, D. C. et al. Models of visuospatial and verbal memory across the adult life span. Psychology and aging 17, 299 (2002).

40 Schaie, K. W. Intellectual development in adulthood: The Seattle longitudinal study. (Cambridge University Press, 1996).

41 Park, D. C. \& Reuter-Lorenz, P. The adaptive brain: aging and neurocognitive scaffolding. Annual review of psychology 60, 173-196 (2009).

42 Hedden, T. \& Gabrieli, J. D. E. Insights into the ageing mind: a view from cognitive neuroscience. Nature reviews neuroscience 5, 87 (2004).

43 Rao, N. \& Wong, J. M. S. (2018).

44 Singh-Manoux, A. et al. Timing of onset of cognitive decline: results from Whitehall II prospective cohort study. Bmj 344, d7622 (2012).

45 Salthouse, T. A. When does age-related cognitive decline begin? Neurobiology of aging 30, 507-514 (2009).

Mufson, E. J. et al. Mild cognitive impairment: pathology and mechanisms. Acta neuropathologica 123, 13-30 (2012).

47 Shankar, G. M. \& Walsh, D. M. Alzheimer's disease: synaptic dysfunction and A $\beta$. Molecular neurodegeneration 4, 48 (2009).

Merienne, N. et al. Cell-Type-Specific Gene Expression Profiling in Adult Mouse Brain Reveals Normal and Disease-State Signatures. Cell reports 26, 2477-2493. e2479 (2019).

49 Sambandan, S. et al. Activity-dependent spatially localized miRNA maturation in neuronal dendrites. Science 355, 634-637, doi:10.1126/science.aaf8995 (2017). Fowler KS, S. M., Conway EL, Semple JM, Louis WJ. . Paired associate performance in the early detection of DAT. . J Int Neuropsychol Soc. 8, 58-71 (2002).

51 Moroney, J. et al. Risk factors for incident dementia after stroke: role of hypoxic and ischemic disorders. Stroke 27, 1283-1289 (1996). Moroney, J. T. et al. Cerebral hypoxia and ischemia in the pathogenesis of dementia after stroke. (1997). Lesne, S. et al. A specific amyloid-beta protein assembly in the brain impairs memory. Nature 440, 284285 (2006).

54 de Fiebre NC, S. N., Forster MJ, de Fiebre CM. Spatial learning and psychomotor performance of C57BL/6 mice: age sensitivity and reliability of individual differences. Age (Dordr). 28, 235-253 (2006). Frick KM, B. L., Arters JA, Berger-Sweeney J. Reference memory, anxiety and estrous cyclicity in C57BL/6NIA mice are affected by age and sex. Neuroscience 95, 293-307 (2009).

56 Verbitsky, M. et al. Altered hippocampal transcript profile accompanies an age-related spatial memory deficit in mice. Learn Mem 11, 253-260 (2004).

57 Jack CR Jr, W. H., Weigand SD, Knopman DS, Vemuri P, Mielke MM, Lowe V, Senjem ML, Gunter JL, Machulda MM, Gregg BE, Pankratz VS, Rocca WA, Petersen RC. . Age, Sex, and APOE $\varepsilon 4$ Effects on Memory, Brain Structure, and $\beta$-Amyloid Across the Adult Life Span. JAMA Neurol. 72, 511-519 (2015). 
58 Panwar B, O. G., Guan Y. miRmine: a database of human miRNA expression profiles. Bioinformatics 33, 1554-1560 (2017).

59 Alles, J. et al. MiR-148a impairs Ras/ERK signaling in B lymphocytes by targeting SOS proteins. Oncotarget 8, 56417-56427, doi:10.18632/oncotarget.17662 (2017).

60 Zheng, B. et al. The function of microRNAs in B-cell development, lymphoma, and their potential in clinical practice. Frontiers in immunology 9, 936 (2018).

61 Chen, L. F. \& Greene, W. C. Shaping the nuclear action of NF-kappaB. Nat Rev Mol Cell Biol. 5, 392401 (2004).

62 Creusot RJ, M. N., Terazzini NM. The immunological synapse. Mol Immunol. 38, 997-1002 (2002).

63 Ortega-Carrion A, V.-M. M. Concerning immune synapses: a spatiotemporal timeline. F1000Res. 31, F1000 Faculty Rev-1418 (2016).

64 Schwartz M, S. R. Systemic inflammatory cells fight off neurodegenerative disease. . Nat Rev Neurol. 6, 405-410 (2010).

65 Molteni M, R. C. Neurodegenerative diseases: The immunological perspective. . J Neuroimmunol 15, 109-115 (2017).

66 Bertagnolo, V. et al. Ectopic expression of PLC- $\beta 2$ in non-invasive breast tumor cells plays a protective role against malignant progression and is correlated with the deregulation of miR-146a. Molecular carcinogenesis 58, 708-721 (2019).

67 Blonska M, L. X. NF- $\mathrm{BB}$ signaling pathways regulated by CARMA family of scaffold proteins. . Cell Res. 21, 55-70 (2011).

68 SC., S. The non-canonical NF- $\kappa$ B pathway in immunity and inflammation. Nat Rev Immunol. 17, 545558 (2017).

69 Ahn, H. J. et al. c-Rel, an NF-kappaB family transcription factor, is required for hippocampal long-term synaptic plasticity and memory formation. Learn Mem 15, 539-549 (2008).

70 Kaltschmidt B, K. C. NF-KappaB in Long-Term Memory and Structural Plasticity in the Adult Mammalian Brain. . Front Mol Neurosci. 24, eCollection 2015 (2015).

71 Jones, S. V. \& Kounatidis, I. Nuclear Factor-Kappa B and Alzheimer Disease, Unifying Genetic and Environmental Risk Factors from Cell to Humans. Front Immunol. 11, eCollection 2017 (2016).

72 Johnson, P. L., Goronzy, J. J. \& Antia, R. A population biological approach to understanding the maintenance and loss of the T-cell repertoire during aging.

. Immunology 142, 167-175 (2014).

73 Ridolfi B, A.-H. H. Neurodegenerative Disorders Treatment: The MicroRNA Role. Curr Gene Ther. Epub ahead of print (2018).

74 Shi M, L. C., Cook TJ, Bullock KM, Zhao Y, Ginghina C, Li Y, Aro P, Dator R, He C, Hipp MJ, Zabetian CP, Peskind ER, Hu SC, Quinn JF, Galasko DR, Banks WA, Zhang J. Plasma exosomal $\alpha$-synuclein is likely CNS-derived and increased in Parkinson's disease. Acta Neuropathol. 128, 639-650 (2014).

$75 \mathrm{Xu}, \mathrm{X} . \mathrm{F}$. et al. miR-181a participates in contextual fear memory formation via activating mTOR signaling pathway. Cerebral Cortex 28, 3309-3321 (2017).

76 Zhang, S.-f., Chen, J.-c., Zhang, J. \& Xu, J.-g. miR-181a involves in the hippocampus-dependent memory formation via targeting PRKAA1. Scientific reports 7, 8480 (2017).

77 Ouyang, Y. B. et al. miR-181 regulates GRP78 and influences outcome from cerebral ischemia in vitro and in vivo. Neurobiol Dis. 45, 555-563 (2012).

78 Moon, J. M., Xu, L. \& Giffard, R. G. Inhibition of microRNA-181 reduces forebrain ischemia-induced neuronal loss. . J Cereb Blood Flow Metab. 33, 1976-1982 (2013).

79 Xu, L. J., Ouyang, Y. B., Xiong, X., Stary, C. M. \& Giffard, R. G. Post-stroke treatment with miR-181 antagomir reduces injury and improves long-term behavioral recovery in mice after focal cerebral ischemia. . Exp Neurol. 26, 1-7 (2015).

80 Saba, R. et al. Dopamine-regulated microRNA MiR-181a controls GluA2 surface expression in hippocampal neurons. Mol Cell Biol 32, 619-632 (2012).

81 Sambandan, S. et al. ctivity-dependent spatially localized miRNA maturation in neuronal dendrites. Science 355, 634-637 (2017).

82 Stepniak, B. et al. Accumulated common variants in the broader fragile X gene family modulate autistic phenotypes. EMBO Mol Med 7, 1565-1579 (2015).

83 Khanna, A., Muthusamy, S., Liang, R., Sarojini, H. \& Wang, E. Gain of survival signaling by downregulation of three key miRNAs in brain of calorie-restricted mice. Aging, 223-236 (2011).

84 Means, L. W., Higgins, J. L. \& Fernandez, T. J. Mid-life onset of dietary restriction extends life and prolongs cognitive functioning. Physiol Behav 54, 503-508 (1993).

85 Dong W, W. R., Ma LN, Xu BL, Zhang JS, Zhao ZW, Wang YL, Zhang X. Influence of age-related learning and memory capacity of mice: different effects of a high and low caloric diet. Aging Clin Exp Res. 28, 303-311 (2016). 
86 Hassenstab, J. et al. Neuropsychological Markers of Cognitive Decline in Persons With Alzheimer Disease Neuropathology. J Neuropathol Exp Neurol. 74, 1086-1092 (2015).

87 Franklin, S. B., Gibson, J. D., Robertson, P. A., Pohlmann, J. T. \& Fralish, J. S. Parallel Analysis: a method for determining significant principal components. Journal of Vegetation Science 1, 102-123 (1995).

88 Hayden, K. M. et al. Factor structure of the National Alzheimer's Coordinating Centers uniform dataset neuropsychological battery: an evaluation of invariance between and within groups over time. Alzheimer Dis Assoc Disord. 25, 128-137 (2011).

89 Stuendl, A. et al. Induction of $\alpha$-synuclein aggregate formation by CSF exosomes from patients with Parkinson's disease and dementia with Lewy bodies. Brain 139, 481-494 (2016).

90 Martin, M. Cutadapt removes adapter sequences from high-throughput sequencing reads. EMBnet. journal 17, pp. 10-12 (2011).

91 Plaisier SB, T. R., Wong JA, Graeber TG. aRnk-rank hypergeometric overlap: identification of statistically significant overlap between gene-expression signatures. Nucleic Acids Res. 38, e169 (2010).

92 Bindea, G. et al. ClueGO: a Cytoscape plug-in to decipher functionally grouped gene ontology and pathway annotation networks. Bioinformatics 25, 1091-1093 (2009). Shannon, P. et al. Cytoscape: a software environment for integrated models of biomolecular interaction networks. Genome research 13, 2498-2504 (2003).

94 Livak, K. J. \& Schmittgen, T. D. Analysis of relative gene expression data using real-time quantitative PCR and the 2- $\Delta \Delta$ CT method. methods 25, 402-408 (2001).

95 Dutta S, S. P. Men and mice: Relating their ages. Life Sci. 1, 244-248 (2016).

96 Li, N., Bates, D. J., An, J., Terry, D. A. \& Wang, E. Up-regulation of key microRNAs, and inverse downregulation of their predicted oxidative phosphorylation target genes, during aging in mouse brain. Neurobiology of aging 32, 944-955 (2011).

97 Inukai, S., de Lencastre, A., Turner, M. \& Slack, F. Novel microRNAs differentially expressed during aging in the mouse brain. PloS one 7, e40028 (2012).

98 Danka Mohammed, C. P. et al. miR-204 downregulates EphB2 in aging mouse hippocampal neurons. Aging cell 15, 380-388 (2016).

99 Gjoneska, E. et al. Conserved epigenomic signals in mice and humans reveal immune basis of Alzheimer's disease. Nature 518, 365 (2015).

100 Zheng, B. et al. PGC-1 $\alpha$, a potential therapeutic target for early intervention in Parkinson's disease. Science translational medicine 2, 52ra73-52ra73 (2010).

101 Ping, L. et al. Global quantitative analysis of the human brain proteome in Alzheimer's and Parkinson's Disease. Scientific data 5, 180036 (2018).

102 Stilling, R. M. et al. K-Lysine acetyltransferase 2a regulates a hippocampal gene expression network linked to memory formation. The EMBO journal 33, 1912-1927 (2014).

103 Kerimoglu, C. et al. KMT2A and KMT2B mediate memory function by affecting distinct genomic regions. Cell reports 20, 538-548 (2017). 


\section{Supplementary Figures}

A

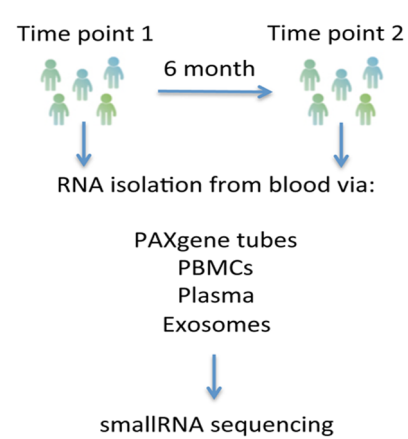

B



E
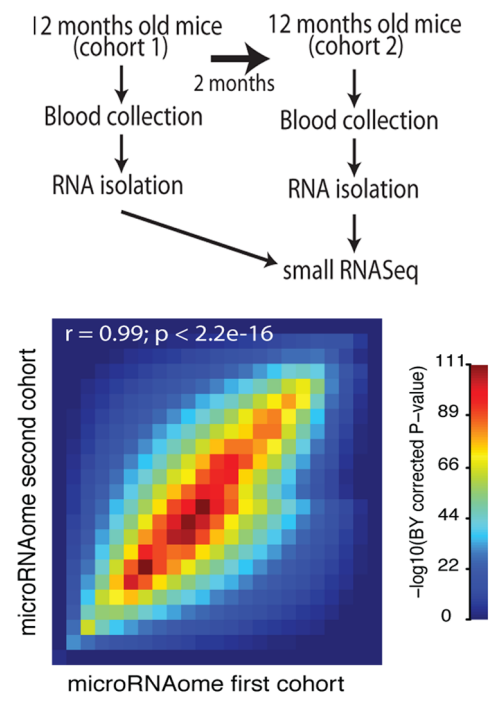

$\mathbf{F}$

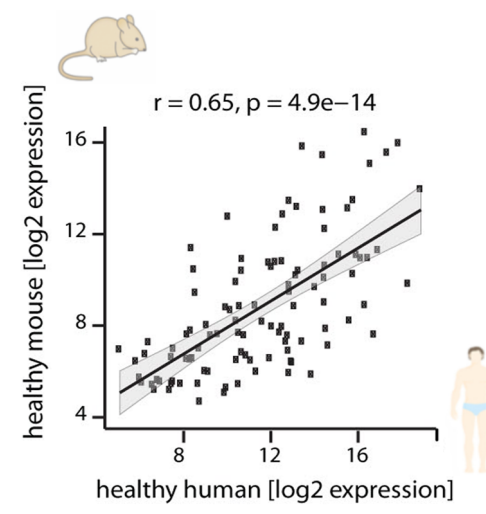

Fig S1. Analyzing circulating microRNAs in mice and humans.

A. Experimental design of the pilot experiment. We analyzed the circulating microRNA expression profiles in blood samples obtained from healthy individuals at two different time points. RNA was isolated from blood using four different approaches, namely via PAXgene tubes (Qiagen), from peripheral blood monocytes (PBMC), plasma, and blood exosomes. B. All samples were subjected to small RNA sequencing. The Venn diagram shows the microRNAs detected via the four different approaches at time point 1 . In total, 125 microRNAs were commonly detected in blood via all four methods. C. Heat map showing the correlation of small RNA-seq data obtained from blood at time point 1 and 2. When comparing the expression of blood microRNAs at time point 1 vs. time point 2 we observed that most consistent results were obtained when small RNA sequencing was performed on RNA isolated via PAXgene tubes or from PBMCs D. Considering that the isolation of RNA from blood via PAXgene tubes is comparatively easy and can also be performed 
in a clinical setting without access to a wet lab we decided to focus on this approach and replicate our observation in an independent experiment. To this end, PAXgene blood samples were collected from an additional group of individuals at two different time points. Also, in this replication cohort, the microRNA expression amongst individuals and amongst time points 1 and 2 was highly correlated as shown by the corresponding heat map. E. According to the manufacturer, the RNAeasy system (Qiagen) for the isolation of RNA from the blood of small animals is based on the same chemistry as the PAXgene (Qiagen) system for human blood collection suggesting that data obtained from humans via PAXgene tubes and mice via the RNAeasy kit would allow for optimal comparison. To test this more specifically, we established a protocol for the longitudinal collection of blood from mice followed by small RNA sequencing (see methods for details). Next, we performed a pilot experiment in mice to test our protocol of blood collection followed by small RNA sequencing. The upper panel depicts the schematic outline of the pilot experiment $(\mathrm{n}=$ 10 /cohort). We collected blood from a cohort of 12 months old mice and store the samples at $-80^{\circ} \mathrm{c}$ (cohort 1). Two months later, the exact procedure was repeated in another cohort of 12 months old mice (cohort 2). RNA samples from cohort 1 and cohort 2 were then subjected to small RNA sequencing. The lower panel shows a rank-rank hypergeometric overlap (RRHO) analysis that revealed that the expression of microRNAs detected in cohort 1 and cohort 2 was highly overlapped (rho $=0.9$ ). Each pixel represents the overlap between two cohorts, and color-coded according to adjusted - $\log 10$ p-value of a hyper-geometric test. F. We tested to what extent the circulating microRNAome defined via the above-described methods in mice is representative to that of humans. To this end we compared samples collected via PAXgene tubes from humans around 40 years of age $(38+/-11$ year, $n=19)$ to samples obtained via the RNAeasy kit from mice at 12 month of age, since 12-month of age in mice is believed to relate to 40 years of age in humans ${ }^{95}$. Our data reveals that the majority of the microRNAs detected in humans PAXgene blood samples is also observed in mice. Even more impressive is the fact that the corresponding expression levels of the circulating human and mouse microRNAome were significantly correlated $(\mathrm{r}=0.65, \mathrm{p}=4.9 \mathrm{e}-14)$. 


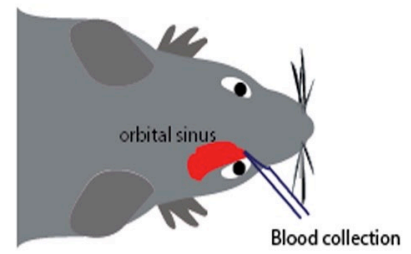

\author{
visual cued assisted \\ water maze task
}



Fig S2. Behavior and sequencing data analysis from longitudinal experiments.

Blood was collected from anesthetized mouse via the retro-orbital sinus. Alternate eyes were used during blood collection at different time points. A visual cue assisted probe test (schematically indicated in the left panel) was performed to check visual performance of mice after habituation training at 12 months (test1, $n=10$ ) and after finishing probe test at 16.5 months (test $2, n=10$ ). In this test mice are able to see the platform and thus do not need to rely on spatial reference memory to find the platform. Compromised vision would impair the performance in this test. There is no significant difference in performance (paired t-test, two-tailed, p value 0.59) between two tests. Error bar indicates standard error mean. 
B
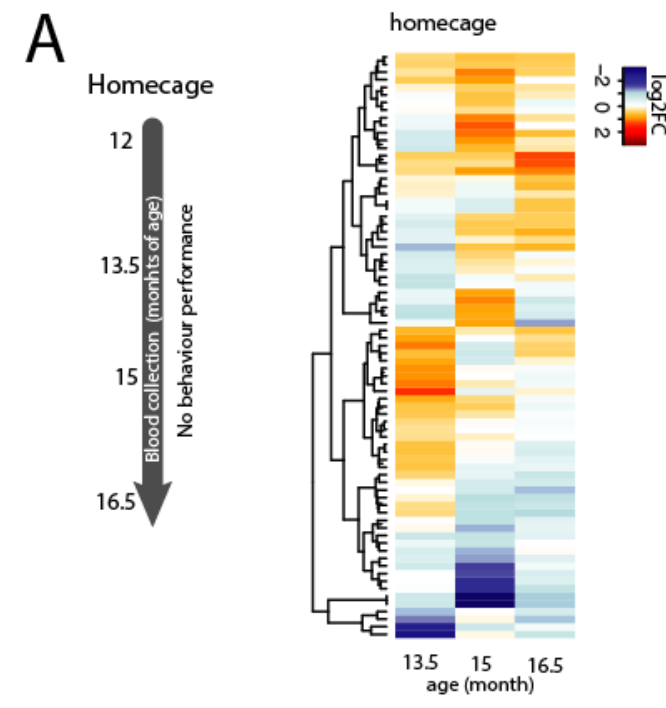

homecage

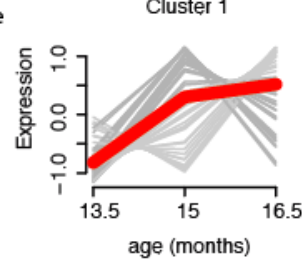

D
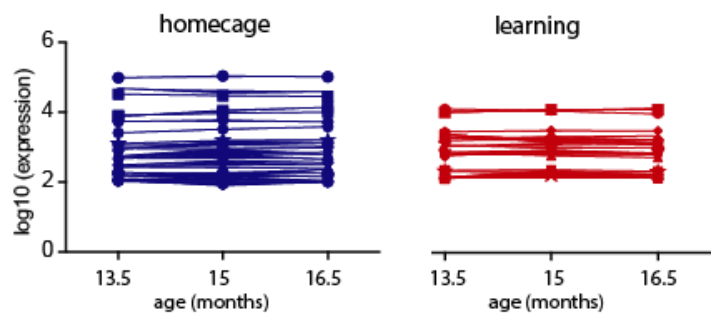

E
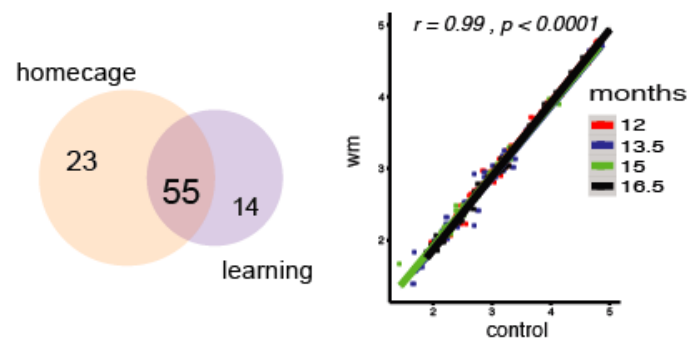

Fig S3. Aging associated microRNA changes in the "home-cage" group

A. Outline of the experiment in home-cage group. Blood was collected at the given time points, but no behavioral test was performed. B. Heat map showing differentially expressed microRNAs during aging in the home-cage group. C. Age-dependent deregulated microRNAs separate into two main clusters: i) upregulated at late age ii) down-regulated at late age. D. Expression cluster of microRNAs that do not change during aging in the homecage and learning group.

E. (Left panel) Venn diagram showing that 55 microRNAs are commonly deregulated during aging in the learning and home-cage groups. (Right panel) The expression of the 55 microRNAs commonly regulated during aging in the home-cage and learning group is highly correlated at all investigated time points $\left(\mathrm{r}=0.99,{ }^{* * * *} \mathrm{p}<0.0001\right)$. 


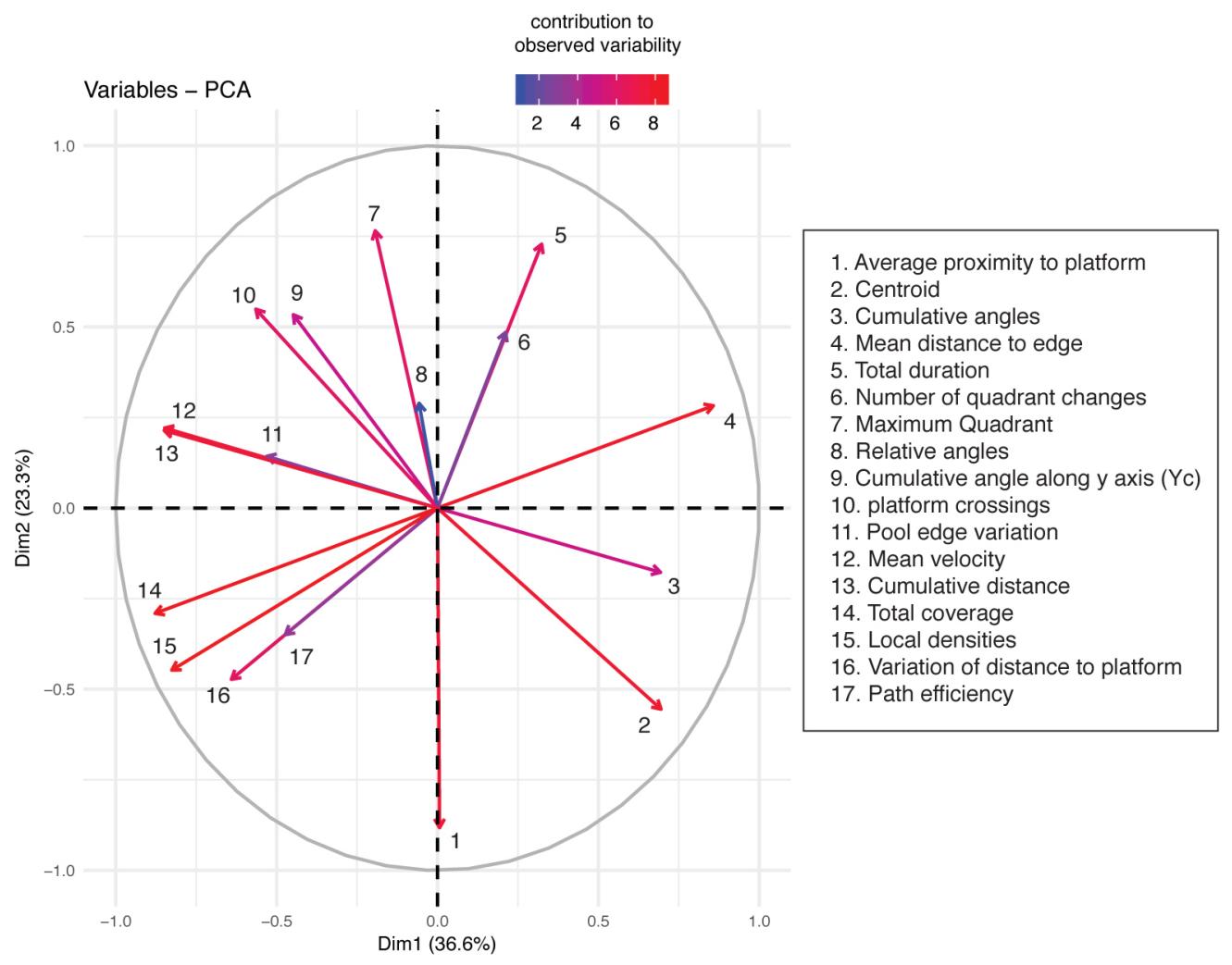

Fig S4. Principle component analysis of the features measured during spatial reference learning in the water maze task.

PCA plot using the seventeen different variables used to measure performance in the water maze test. First two components (PC1 and PC2) could explain sixty percent of the variation in data. The length of the arrows indicates how much variability is explained by each of the 17 learning strategies. 
A
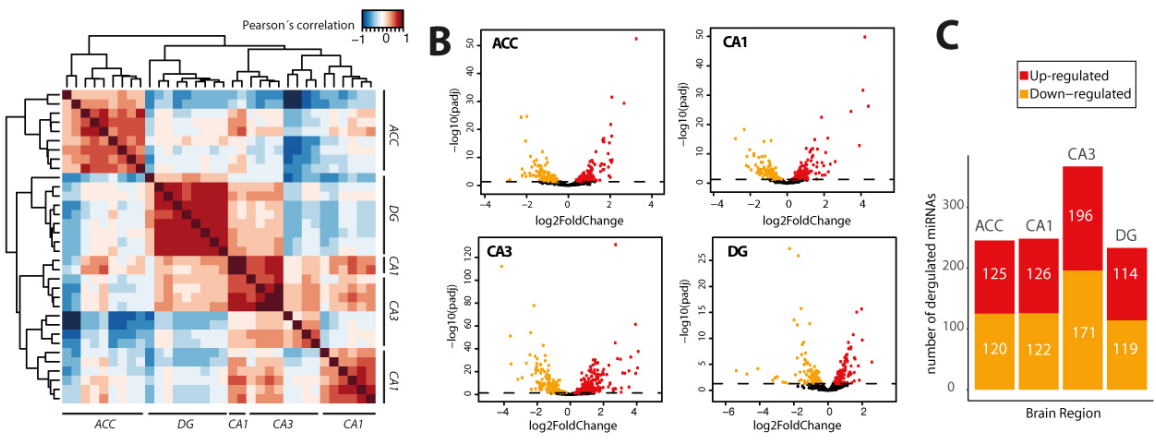

D

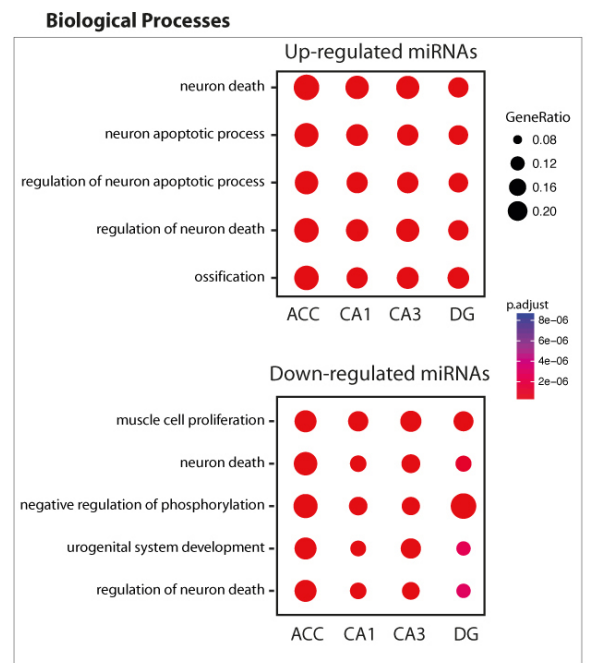

KEGG pathways



$\mathbf{E}$
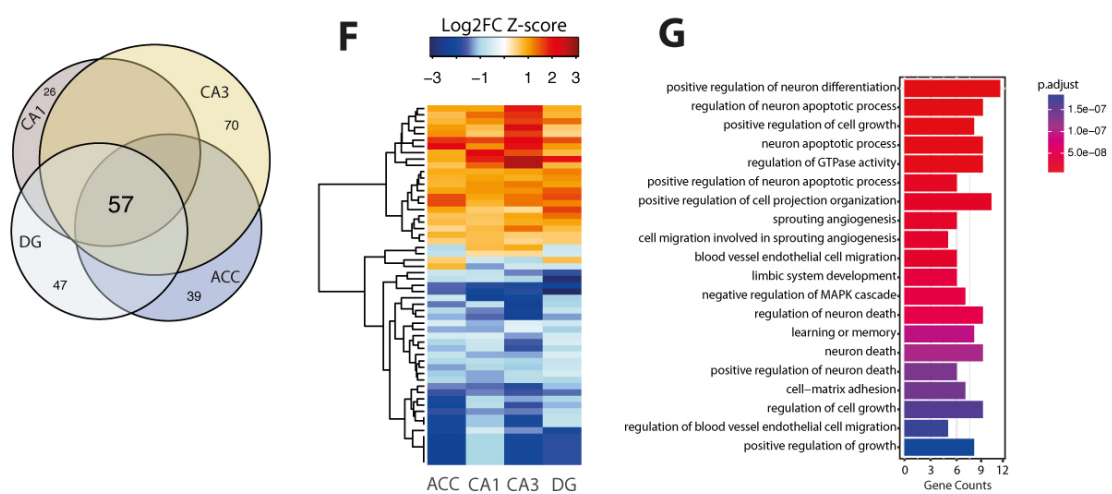

Fig S5. The microRNAome of learning-related brain regions in the aging mouse brain

Previous genome-wide microRNAome data on brain aging ${ }^{96-98}$ are characterized by low sample size, include array approaches limited by probe design and focused on whole brain or whole hippocampal tissue. Thus, we performed small RNA sequencing of the hippocampal sub-regions CA1, CA3 and dentate gyrus (DG) and the anterior cingulate cortex (ACC) isolated from young (3 months of age) and cognitively impaired old (18 months of age) mice. A. Pearson's coefficient-based correlation followed by unsupervised clustering of all small RNA-seq data from different brain regions (ACC; CA1; CA3; DG) in young mice reveals brainregion specific expression of the microRNAs that was particularly obvious for the ACC vs. the hippocampal sub-regions and the DG vs. CA1 and CA3. These region-specific differences were, however, mainly attributed to different expression values since the majority of the microRNAs, namely 176 microRNAs, could be detected at reliable levels in all investigated brain regions (see also Table S4). B. Volcano plots showing differential expression of microRNAs in the different brain regions when comparing young vs. old mice. C. Bar plots showing the number of up and down-regulated microRNAs in the investigated brain regions. D. Comparison of gene ontology and functional pathways of experimentally confirmed target genes of the deregulated microRNAs in the investigated brain regions. Pathways are generally linked to neuronal death and longevity pathways. E. Venn diagram showing that 57 microRNAs are commonly deregulated in the aging brain. F. Heat map showing hierarchical clustering of the 57 commonly deregulated microRNAs 
based on Log2 fold change $\mathrm{Z}$ score. G. Top 20 biological processes affected by the confirmed target genes of the 57 commonly deregulated microRNAs in the aging brain.
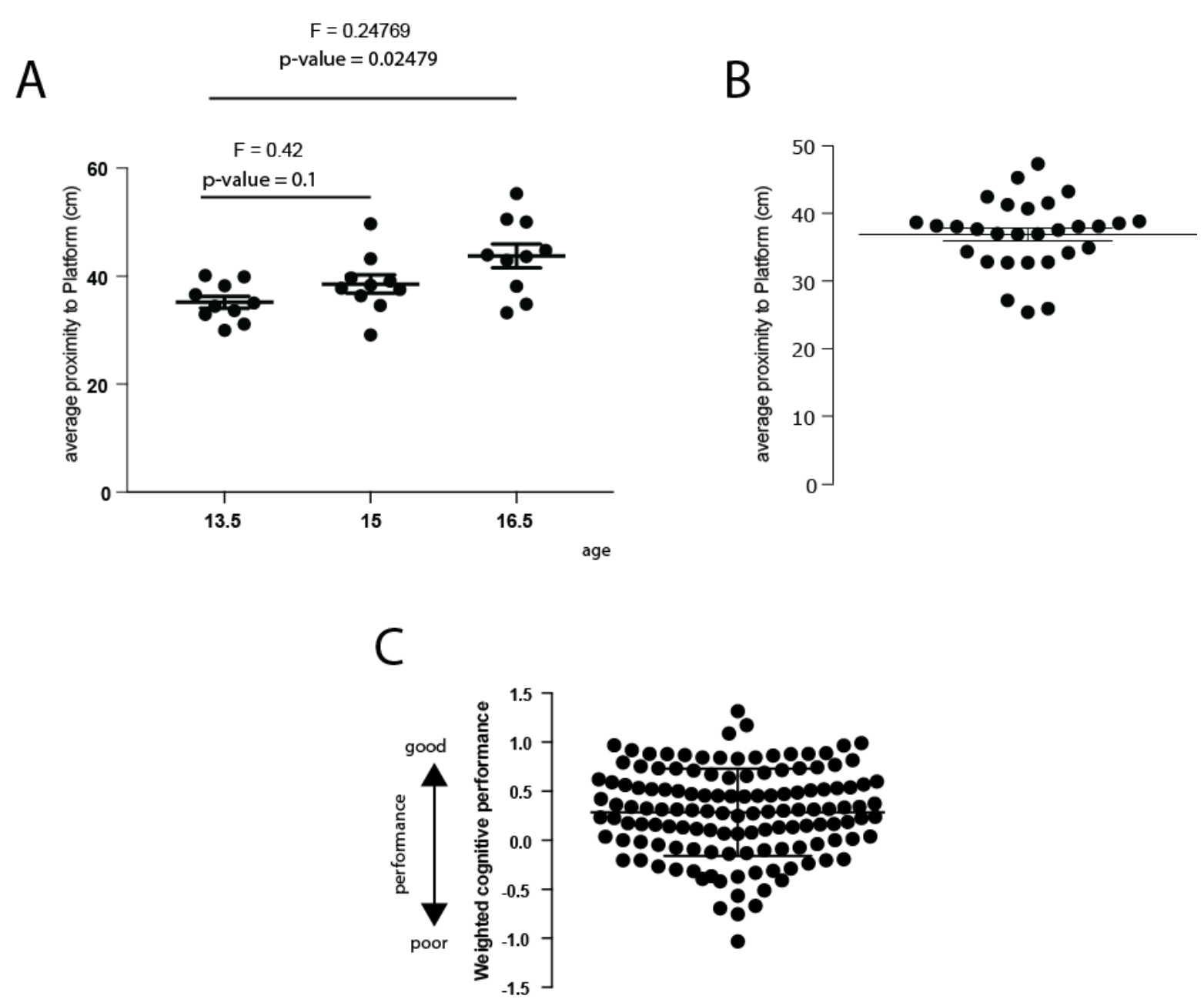

Fig S6. Inter-individual variability in mouse and healthy human individuals

A. With increasing age, the inter-individual variability in cognitive function, displayed here as average proximity to platform during the water maze probe test increases $(n=10 ; \mathrm{F}$ test, $\mathrm{p}=0.02 ; 16.5$ months compared to 13.5 months). Variance is calculated using var.test function in R. Error indicate SEM. B. Variability in average proximity was noted in an independent cohort of 16.5 months old mice $(n=29) \mathrm{C}$. The data depicts the variability in cognitive performance amongst the control individuals of the PsyCourse cohort $(n=132)$. Cognitive score was calculated on the basis of 8 neuropsychological tests (See methods for details). The variability in cognitive abilities allowed us to test the correlation of microRNAs in blood with cognitive function. 


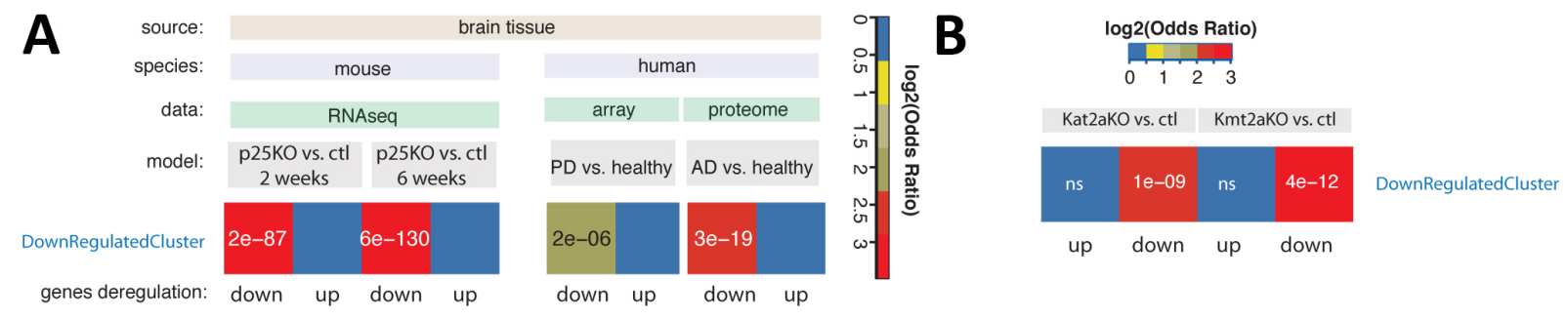

Fig S7. microRNA-181a regulating conserved gene network in different models of memory disorders.

A. microRNA-181a-5p regulating cluster "DownRegulatedCluster" is further down-regulated in brain of CKp25 mice ( 2 weeeks and 6 weeks), model of AD pathology ${ }^{99}$. Besides mice, the same cluster of genes is downregulated in human subjects with PD (Parkinson's Disease) ${ }^{100}$. Intriguingly, proteins of the genes belonging to "DownmiR181aTargetCluster" were also downregulated in AD human compared to neurologically healthy subjects ${ }^{101}$. B. "DownRegulatedCluster" is also significantly down-regulated in Kat2aKO ${ }^{102}$ and $\mathrm{Kmt}^{2} \mathrm{aKO}^{103}$ mice compared to the corresponding controls. Color bar is indicating the Odds ratio in $\log 2$ scale that measures the strength of overlap between two lists. $\log 2$ odds ratio $\leq 0$ indicates no overlap while the greater the value than 0 , the stronger overlap between two datasets is present. Numbers in the boxes represent adjusted $p$ value of hypergeometric test.

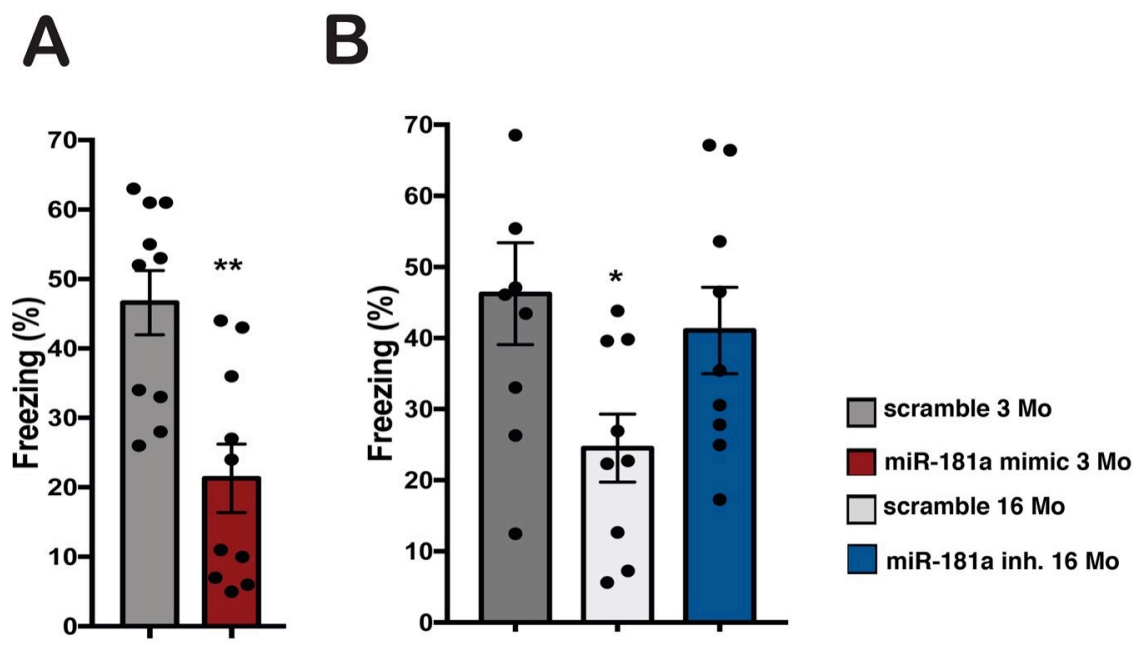

Fig S8. microRNA-181a-5p regulates consolidation of associative fear memories

A. 3 months old mice (3 Mo) were injected with either microRNA-181a-5p (microRNA-181a) mimic or scrambles control oligonucleotides into the dorsal hippocampus and subsequently subjected to contextual fear conditioning. During the memory test performed $24 \mathrm{~h}$ later mice injected with the microRNA-181a-5p mimic displayed significantly less freezing behavior indicate of impaired memory function $(\mathrm{p}<0.05 \mathrm{vs}$. scrambled control, $t$-test). B. A microRNA-181a-5p inhibitor oligonucleotide was injected in into the dorsal hippocampus of aged mice (16 month of age, 16Mo). A scramble control oligonucleotide injected into the hippocampus of 3- and 16-months old mice was used as control. ANOVA revealed a significant difference amongst groups $(p=0.04)$. While freezing behavior was impaired when comparing scramble-injected 3 vs. 16 months old mice $(p=0.02)$, no such difference was observed when the group of 16 months old mice that received microRNA-181a-5p inhibitor were compared to the scramble-injected 3 months old mice $(p=0.6)$. Error bars indicate SEM. 


\begin{tabular}{|c|c|}
\hline &  \\
\hline mouse & AACATTCAACGCTGTCGGTGAGT \\
\hline human & AACATTCAACGCTGTCGGTGAGT \\
\hline Rat & AАCATTCAACGCTGTCGGTGAGT \\
\hline Chimpanzee & AACATTCAACGCTGTCGGTGAGT \\
\hline Rhesus_Macaque & AACATTCAACGCTGTCGGTGAGT \\
\hline Bonobo ${ }^{-}$ & AACATTCAACGCTGTCGGTGAGT \\
\hline Gorilla & AACATTCAACGCTGTCGGTGAGT \\
\hline Zebrafish & АACATTCAACGCTGTCGGTGAGT \\
\hline Chicken & AACATTCAACGCTGTCGGTGAGT \\
\hline pig-tailed macaque & AACATTCAACGCTGTCGGTGAGT \\
\hline orangutan & AACATTCAACGCTGTCGGTGAGT \\
\hline Wolly_Monkey & AACATTCAACGCTGTCGGTGAGT \\
\hline & $* * * * * * * * * *$ \\
\hline & ed regio \\
\hline
\end{tabular}

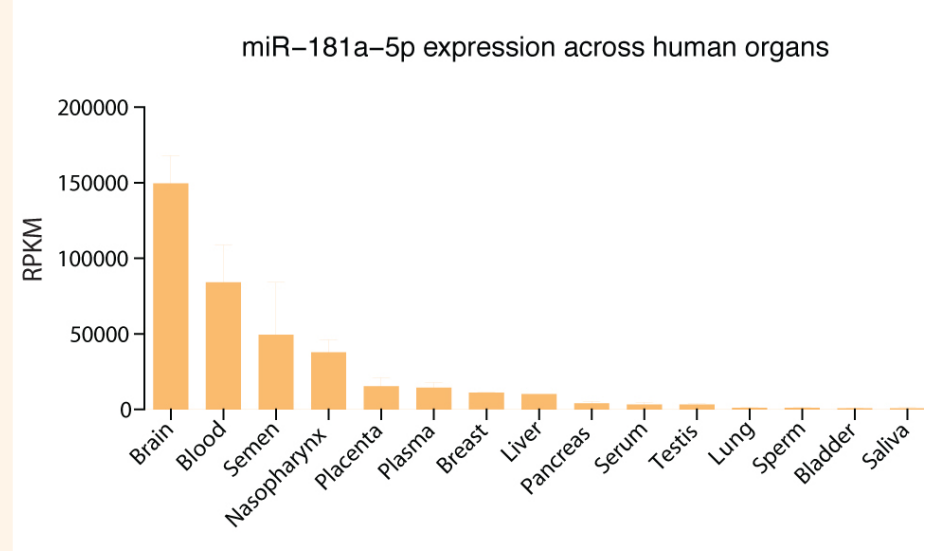

Fig S9. mciroRNA-181 is highly conserved across species.

Left panel: Multiple sequence alignment of microRNA-181a-5p mature sequences from different species. microRNA-181a-5p seed sequence is conserved across different species. Right panel: microRNA-181a-5p expression across different human tissues based on miRMine Database. Note that microRNA-181a-5p is highest expressed in brain and blood. 


\section{Research Article 2 (prepared for submission) \\ Epigenetic modifications underpin heart failure associated cognitive deficits}

Md Rezaul Islam ${ }^{1}$, M Sadman Sakib ${ }^{1}$, Raoul Maximilian Hofmann², Dawid Lbik², Tea Berulava $^{1}$, Martí Jiménez Mausbach ${ }^{1}$, Susanne Burkhardt ${ }^{1}$, Alessya Kretzschmar ${ }^{2}$, Julia Cha ${ }^{1}$, Elerdashvili Vakhtang ${ }^{1}$, Dennis M Krueger ${ }^{1}$, Farahnaz Sananbenesi ${ }^{4}$, Karl Toischer ${ }^{2,3}$, Andre Fischer $^{1 *}$ 


\section{Epigenetic modifications underpin heart failure associated cognitive deficits}

Md Rezaul Islam¹, M Sadman Sakib', Raoul Maximilian Hofmann², Dawid Lbik ${ }^{2}$, Tea Berulava $^{1}$, Martí Jiménez Mausbach ${ }^{1}$, Susanne Burkhardt ${ }^{1}$, Alessya Kretzschmar ${ }^{2}$, Julia Cha ${ }^{1}$, Elerdashvili Vakhtang ${ }^{1}$, Dennis M Krueger ${ }^{1}$, Farahnaz Sananbenesi ${ }^{4}$, Karl Toischer ${ }^{2,3}$, Andre Fischer $^{*}$

${ }^{1}$ German Center for Neurodegenerative Diseases (DZNE), Von Siebold Str. 3a, 37075, Göttingen, Germany

${ }^{2}$ Clinic of Cardiology and Pneumology, Georg-August-University, Göttingen, Germany ${ }^{3}$ German Centre for Cardiovascular Research (DZHK), partner site Göttingen, Germany ${ }^{4}$ Department of Psychiatry and Psychotherapy, University Medical Center Göttingen, Germany

*To whom correspondence should be addressed; andre.fischer@dzne.de 


\section{Abstract}

Mounting evidence suggests the co-occurrence of cardiac failure and dementia. The mechanisms that drive cardiac failure-induced cognitive decline and thereby increase the risk for dementia remain, however, largely unexplored. Moreover, therapeutic intervention for managing cognitive impairment in heart failure patients is missing. In this study, we analyze the neuronal function and memory performance in a mouse model for cardiac hypertrophy. We observed that mice in which cardiac-specific CamkII $\delta \mathrm{c}$ is overexpressed in the heart exhibit massive alterations in hippocampal gene-expression that resemble changes observed in neurodegenerative diseases. Up-regulated genes represent endoplasmic reticulum-stress (ER)stress, while down-regulated genes are linked to neuronal function, histone-methylation, and synaptic plasticity. In line with these data, CamkII $\delta$ c mutant mice exhibit memory impairment that was associated with reduced neuronal histone 3 lysine 4 tri-methylation (H3K4me3), a key euchromatin mark implicated with active gene expression. Oral administration of Vorinostat (SAHA), an epigenetic drug that promotes euchromatin formation, was able to reinstate memory function in CamkII $\delta$ c mutant mice via the elevation of H3K4me3-related reexpression of synaptic plasticity genes. In addition, SAHA regulated a brain-specific microRNA-network that counteracted ER-stress-related gene-expression. Our study provides the first insight into the molecular processes that influence brain function as a result of cardiac diseases and suggests that epigenetic strategies could be suitable to restore cognitive function in heart failure patients and attenuate the risk to develop dementia.

Key words: Heart failure, cognitive decline, epigenetic regulation, SAHA as therapy, microRNAs 


\section{Introduction}

Heart failure (HF) and dementia share similar risk factors including vascular factors, age, lack of education, and gender $^{1-3}$. Reduced cardiac contractility and dysfunctions in cardiac perfusion are functional characteristics of heart failure patients. Studies investigating brain anatomy in heart failure patients reported reduced cerebral blood flow in HF brains compared to that of healthy controls ${ }^{4}$. Regional hypoperfusion due to the failing heart may result in functional and structural alterations of the brain. In a recent study, hypoperfusion in both prefrontal cortex and hippocampus along with other regions has been reported in HF patients ${ }^{4}$. Moreover, cerebral structural alterations have been described in both white ${ }^{5}$ and gray matter ${ }^{6}$ as well as in different brain regions, including the cortex and hippocampus. Furthermore, another study reported reduced regional cortical thickness in HF patients that may be attributed to the loss of different brain cells ${ }^{6}$. Concordantly, recent functional imaging data revealed tissue loss in frontal cortex ${ }^{7}$ and hippocampus ${ }^{8}$ of HF patients.

Since these are brain regions of critical functions, hippocampus in particular, and are inherently linked to learning and memory, structural damage in these regions is likely to affect cognition. In agreement with this, mounting evidence shows that heart failure can be associated with cognitive impairment ${ }^{1,9,10}$. A high prevalence of early-onset cognitive deficits ranging between $25 \%-75 \%$ has been reported in patients with failing heart ${ }^{11-14}$. Despite such high prevalence, (i) the underlying molecular changes occurring in the brain as a result of heart failure are poorly understood and (ii) therapeutic strategies for the management of cognitive impairment in HF patients are missing.

A recent study pointed out a possible association between improvements in cardiovascular health with a lower incidence of dementia ${ }^{1}$. However, drugs (e.g., $\beta$-blockers, digitalis, aldosterone antagonists) that have been used in HF patients lack evidence for improving cognition ${ }^{15-17}$. Although ACE inhibitors showed some potentials to benefit cognition in $\mathrm{HF}^{18}$, contrasting results from other study demonstrate its association with an increased incidence of Alzheimer's disease ${ }^{19}$. Concerns regarding other novel agent such Subcubitril/Valsartan and its potential to promote Alzheimer's disease is not yet eliminated ${ }^{20,21}$. Also, other heart failure therapies e.g. left ventricular assist devise (LVAD), veno-arterial extracorporeal membrane oxygenation (VA-ECMO) have been associated with cognitive impairment ${ }^{13}$. Therefore, proven therapeutic options to ameliorate cognitive impairment in HF are absent, which is most 
likely due to the fact that the mechanistic insight that underlies the cognitive changes following reduced perfusion in the brain is missing. Consequently, the European Society of Cardiology (ESC) recently recommend joint efforts of experts from cardiology and dementia research to investigate therapeutic interventional options for managing cognitive impairment in subjects with cardiac failure ${ }^{22}$.

In this study, we employ a mouse model of cardiac hypertrophy and heart failure to study the mechanisms that lead to altered brain function and memory impairment. Our results showed that cardiac hypertrophy leads to massive changes in hippocampal gene expression linked to increased stress responses (e.g., oxidative, endoplasmic), protein misfolding, autophagy, and decreased neuronal plasticity. These changes in gene expression are partly linked to epigenetic modifications and a loss of euchromatin. In line with this, HF mice exhibited cognitive deficits. Aberrant gene expression and memory impairment were ameliorated via the oral treatment of Vorinostat (SAHA), an epigenetic drug that promotes euchromatin formation. SAHA also induced a microRNA network that counteracted the detrimental expression of ER-stress related genes. To the best of our knowledge, this is the first study that elucidates the molecular changes in the hippocampus in the context of cardiac hypertrophy and heart failure. Moreover, we propose that epigenetic therapeutic intervention may help in clinical settings to improve cognition in HF patients. 


\section{Results}

\section{CamkIISc overexpression in heart leads to brain gene expression changes similar to neurodegenerative diseases}

Since cardiovascular risk factors can negatively influence overall brain health ${ }^{23,24}$, we used a heart failure (HF) transgenic mouse model to investigate the effect of faulty heart in shaping brain functions. Transgenic mice were bred according to previous study ${ }^{25}$ and they overexpressed CamkII $\delta$ c exclusively in the heart owing to alpha-MHC promoter upstream of its transgene (Fig 1A). At the age of 3 months, these mice displayed shrinkage of the lumen volume (Fig 1B), increased systolic as well as diastolic diameter (Fig 1C) in the left ventricle and lower ejection fraction rate (Fig 1D) compared to age-matched littermate controls. These data imply that compared to controls, normal cardiac function is substantially reduced in transgenic mice. Although transgenic mice displayed similar body weight (Fig 1E), their heart weight (Fig 1F) was substantially increased compared to controls. Consistently, heart to body weight (Fig 1G) ratio and particularly the weight of the left ventricle in the heart (Fig 1H) were significantly higher in transgenic mice. However, we failed to observe significant differences in terms of tibia length (Fig S1A), lung to body weight (Fig S1B) and lung weight/tibia length ratio (Fig S1C) between groups. Hypertrophic heart and reduced cardiac functions as observed in this transgenic mice corroborate previous findings from other studies ${ }^{25,26}$. CamkII $\delta \mathrm{c}$ overexpression dependent prolonged ryanodine receptor (Ryr2) activation followed by sarcoplasmic reticulum calcium leakage underpin reduced contractile function observed in these transgenic mice ${ }^{25,26}$. Recent studies have shown that cardiac disruptions in ryanodine receptors and defective $\mathrm{Ca}^{2+}$ signaling in the myocardium can exacerbate endoplasmic stress, protein misfolding, oxidative response and autophagy in the hearts of cardiac failure patients ${ }^{27}$. Recent attempts to understand detailed genetic interactions in heart failure have led studies investigating cardiac transcriptomics from healthy and disease conditions ${ }^{28-30}$. We analyzed one of these data ${ }^{30}$ and found that indeed, heart failure leads to significant dysregulations of cardiac transcriptomics (Supplementary Table 1a, Fig S1D) compared to those of healthy controls. Interestingly, down-regulated genes could be involved in different biological and molecular processes, including heart contraction and heart process as expected (Fig S1E). Moreover, they are also likely to contribute to protein folding and RNA splicing (Fig S1E, Supplementary Table 1b). 

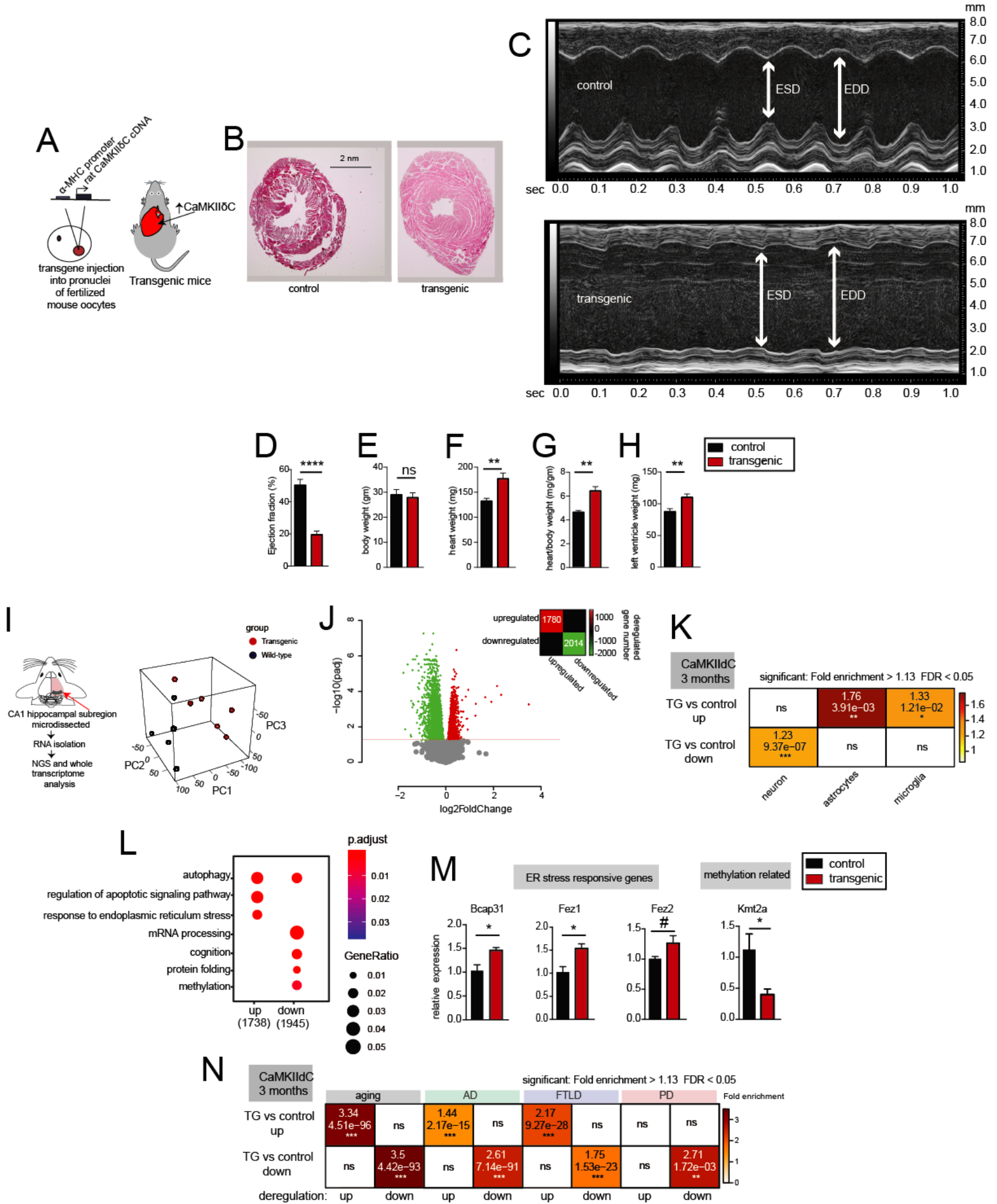

Fig 1. Mice overexpressing CamkIIסc display ER stress response in the molecular level.

A) scheme of mice overexpressing CamkII $\delta \mathrm{c}$ in the hearts of the transgenic mice. B) Immunostaining for the left ventricle tissue sections and C) M-mode images from left ventricle from wild-type and transgenic mice. ESD: Left ventricle end systolic diameter, EDD: Left ventricle diastolic diameter. D) Ejection fraction is significantly decreased to $\sim 50 \%$ in transgenic mice $(n=8)$ compared to control mice $(n=5)$. (E) CamkII $\delta$ c transgenic mice have similar body weight to transgenic control. Weight of heart (F), heart to body $(\mathrm{G})$ and left ventricle $(\mathrm{H})$ are increased in transgenic mice $(\mathrm{n}=8)$ compared to control $(\mathrm{n}=5)$. (I) Scheme of the sequencing experiment. Principle component analysis (PCA) plot of transcriptomic data shows that transgenic mice $(n=6)$ are different 
from control mice $(n=5)$ in terms of gene expression. First principle component (PC1) can explain $42 \%$ of the variation between the two groups. ( $\mathrm{J}$ ) Volcano plot showing differentially expressed genes (FDR $<0.05)$. Red color indicates upregulation, while blue represents the downregulation of transcripts. Heatmap showing the number of genes deregulated in transgenic mice compared to age-matched littermate controls. K) Hypergeometric overlap analysis taking genes deregulated in transgenic mice and comparing it to genes uniquely expressed in neuron, astrocytes, and microglia. Color represents the fold enrichment. Values in the box (top to bottom) represent fold enrichment, hypergeometric overlap p-value and asterisks based on the significance level L) Top GO biological processes after removing redundant GO terms using Rivago, are summarized in a dot plot. M) qPCR quantification of Bcap31, Fez1, Fez2 in control and transgenic mice ( $n=5$ each group). Quantitative PCR for Kmt2a between transgenic $(n=4)$ and littermate controls $(n=5) .{ }^{*} p<0.05$, Unpaired t-test; two-tailed. Data is normalized to Hprt1 expression. N) Overlap between deregulated genes in CamkII $\delta$ c transgenic mice with those from aging, AD, FTLD, and PD datasets. $\# \mathrm{p}=0.08,{ }^{*} \mathrm{p}<0.05,{ }^{* *} \mathrm{p}<0.01,{ }^{* * *} \mathrm{p}<0.001$. Unpaired t-test; two-tailed. Error bars indicate mean \pm sem

We asked whether cardiac dysfunctions could affect similar molecular processes in brain. To investigate this question, we micro-dissected the CA1 hippocampal sub-region from 3 months old transgenic and control mice and subjected its RNA to bulk RNA sequencing (Fig 1I). Interestingly, the first principle component showed distinct transcriptomic profiles between the two groups (Fig 1I). Differential expression analysis revealed 3794 genes as significantly deregulated between groups (FDR <0.05). Of these, 1780 genes were up-regulated whereas 2014 genes were down-regulated in transgenic mice when compared to the control group (Fig. 1J, S1E, Supplementary Table 2a). Since CamkII $\delta$ expression in brain (FDR 0.52, Supplementary Table 3) did not change between groups, the observed changes in brain gene expression as observed can be attributed to CamkIIIc overexpression in heart and associated hypoperfusion.

A previous study reported that the neuro-vascular unit in the brain, composed of different cell types including but not limited to neurons, microglia, and astrocytes, regulates vascular recruitment in response to local oxygen demands and functional activity in brain ${ }^{31}$. We reasoned that these different cell types in the brain may respond to hypoperfusion induced molecular changes differently. In fact, we and others have shown that peripheral insults can lead to distinct transcriptomic changes in different brain cell types ${ }^{32-34}$ to shape brain function. To determine whether the deregulated genes in CamkII $\delta$ c mice would reflect any cell typespecific enrichment in brain, we analyzed deposited sequencing data from neuron, microglia, and astrocytes ${ }^{35}$. Hypergeometric test revealed that up-regulated genes overlapped significantly with astrocytes and neurons (Fig 1K). In contrast, down-regulated genes showed significant enrichment only in neuron (Fig 1K). 
Investigating the functional relevance of these deregulated genes, we found the down-regulated genes are likely to be involved in mRNA processing (Fig 1L, Supplementary Table 2b, 2c), especially RNA splicing and protein folding. The downregulation of neuron-specific splicing factors in CamkII $\delta$ c transgenic mice (Fig S1G) may hint at its potential causal effect on reduced splicing. Of note, similar biological processes are down-regulated in the hearts of cardiac failure patients (Fig S1E). These data suggest that homologous molecular responses could interplay between the heart and brain in HF model. In addition, genes related to cognition and methylation processes are down-regulated in transgenic mice (Fig 1L). In agreement with this, insulin signaling, mTOR signaling, AMPK signaling, and MAPK signaling pathways related to cognition ${ }^{36-39}$ are down-regulated as well in transgenic mice (Fig $\left.\mathrm{S} 1 \mathrm{H}\right)$.

On the contrary, up-regulated genes are likely to be autophagy, apoptotic signaling, and endoplasmic reticulum (ER) stress-related (Fig. 1L, supplementary Table 2b, 2c). qPCR data confirmed the increased expression of a few selected ER stress related genes (Fez1, Fez2, Bcap31) as well as the decline of Kmt2a expression, a writer of histone modification, in transgenic mice compared to controls (Fig 1M). A previous study has showed that loss of $\mathrm{Kmt} 2 \mathrm{a}$ in the brain leads to cognitive impairment ${ }^{40}$. Increased unfolded protein and ER stress response, along with decreased cognition, have been previously reported in various neurological diseases ${ }^{41}$. Therefore, we asked whether the gene expression changes as observed in this study would overlap with those of other neurodegenerative diseases. To this end, we retrieved multiple datasets related to aging ${ }^{42}$, analyses of Alzheimer's (AD) ${ }^{43}$, Parkinson (PD) ${ }^{44}$ and Fronto-temporal dementia (FTLD) ${ }^{45}$ disease-specific differentially expressed genes compared to corresponding controls. Subsequently, we performed gene enrichment analyses of de-regulated genes from CamkII $\delta$ c transgenic mice and deregulated genes from each of the aforementioned diseases. In fact, we investigated up- and down-regulated genes separately. Interestingly, up-regulated genes in CamkII $\delta \mathrm{c}$ transgenic mice representing stress responses (Fig 1L) significantly overlap with up-regulated genes in all the above diseases with exception in PD (Fig 1N). On other hand, down-regulated genes representing cognition and splicing are overlapped with those from advanced aging, AD, FTLD, and also PD (Fig 1N). These data suggest that, CamkII $\delta$ c transgene overexpression can contribute to upregulation of stressresponsive genes along with the downregulation of cognition related genes in the mouse brain akin to neurodegenerative diseases. 


\section{Heart failure model mice display cognitive impairment at an early age}

Given that we investigated the brain transcriptomic profile of HF mice at an early age (3 months), observing similar gene profile changes associated with diseases characterized by aging and memory loss was intriguing and promoted us to investigate cognition in the transgenic mice. To this end, we employed behavioral experiments that assay spatial reference memory in mice (Fig 2A). Before cognitive performance evaluation, first, we tested whether anxiety-related responses would vary between groups. In this regard, 3 months old transgenic and control mice were subjected to the open field test (Fig 2A). Our results showed that both transgenic $(n=16)$ and control $(n=13)$ mice spent a similar percentage of time in the middle region of the test field, traveled similar paths and moved at almost identical speeds (Fig 2B). These data demonstrated that cognitive performance would be unlikely to be affected by a basal level difference in anxiety. Subsequently, mice were subjected to the Barnes maze, a spatial navigation learning test that evaluates cognitive performance in mice by utilizing environmental cues and an escape hole (Fig S2). TWO-way ANOVA analyses revealed that transgenic mice required significantly more time to find an escape hole compared to littermate controls (Fig 2C). A further detailed analysis considering head angles and trajectories (Fig S2B) employed by mice during learning of the task showed that transgenic mice used strategies that are less hippocampal-dependent (direct, short and long chaining strategic approach) compared to controls (Fig. 2D). Consistently, cumulative strategy scores calculated based on hippocampal-dependent strategies (see methods for details) revealed a significant difference between groups (Fig 2E), confirming that transgenic mice were poor performers in training trials. Next, a memory retrieval test was performed by closing the escape hole. During the retrieval phase, visits to the escape hole were enumerated. Transgenic mice visited the target hole less frequently than did controls (Fig S2F), suggesting an impairment of memory retrieval in transgenic mice (Fig 2F). 


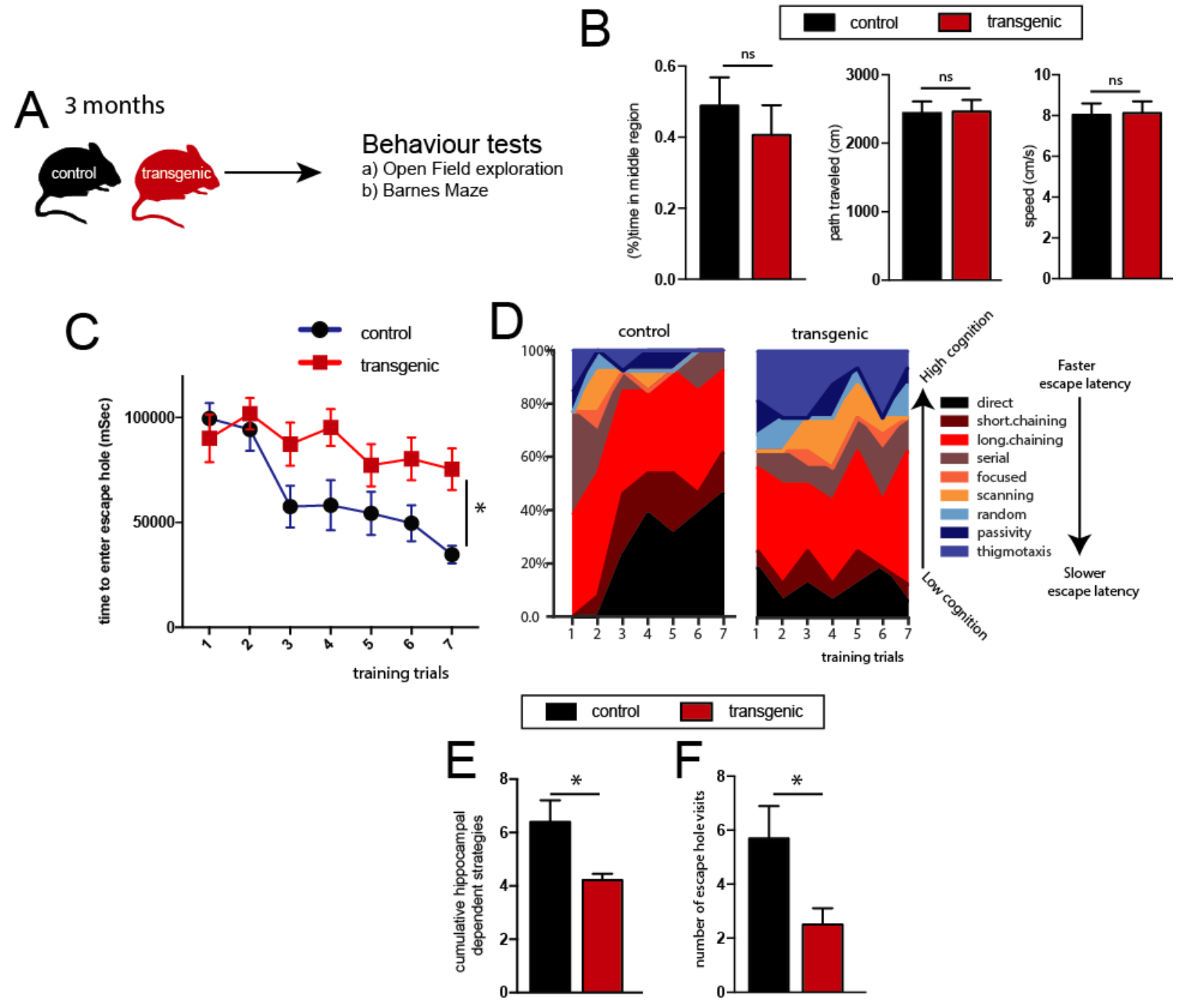

Fig 2. Behavior data at 3 months of age.

A) Behavioral tests (e.g. open field, Barnes maze) were performed with transgenic $(n=16)$ and control mice $(n=$ 13) at 3 months of age. B) Percentage of time spent in middle region (left panel), traveling path (middle panel) and speed (right panel) during test in both groups (two-tailed, unpaired t-test) C) Escape latency in control and transgenic mice (two-way ANOVA, ${ }^{*} \mathrm{p}<0.05$ ) D) Different strategies based on mice trajectories across trials. Each strategy is labelled with a unique color. E) Cumulative strategy scores across training trials and F) number of visits in escape hole during probe test (right panel) [two-tailed, unpaired t-test, ${ }^{*} \mathrm{p}<0.05$ ). Error bar indicates mean \pm sem

In summary, these data demonstrate that CamkII $\delta$ c overexpression-based heart failure model mice display stress-independent cognitive deficits at an early age (e.g., 3 months). 


\section{Histone modifications may underpin cognitive deficits}

Since our functional enrichment results based on down-regulated genes previously pointed to downregulation of methylation in transgenic mice (Fig 1L), we reasoned that epigenetic changes might underpin the cognitive deficits (Fig 2) in transgenic mice. To gain more confidence in this, we decided to compare deregulated genes from our transgenic mice with those from other studies wherein different histone modifier enzymes were genetically manipulated, and their effects on cognition was examined. Thus, we retrieved and analyzed RNA-seq data from Kmt2a ${ }^{46}, \mathrm{Kmt}_{2} \mathrm{~b}^{40}$, and Kat $2 \mathrm{a}^{47}$ knockout experiments. Of note, significantly deregulated genes (FDR $<0.05$ ) were determined from comparisons with littermate controls from each study ${ }^{40,46,47}$. Cognition was impaired in all of these transgenic mice, mainly due to the downregulation of synaptic plasticity-related genes of neurons $\mathbf{s}^{40,46,47}$ (Supplementary Table 3a-c). Interestingly, both up- and down-regulated genes overlap with corresponding those from kmt2a knockout only (Fig 3A). Moreover, overlap between downregulated genes from our transgenic mice and those from Kmt2a knockout experiments (5.1 fold, FDR 1.45e-73) outnumbers other enrichments (Fig 3A). Surprisingly, no significant enrichment was observed with genes deregulated in Kmt2b knockout mice (Fig 3A). These data suggest that changes in gene expression in our heart failure model could be, at least in part, dependent on reduced Kmt2a level. Indeed, we confirmed a substantial reduction of Kmt2a (Fig 2B) expression in transgenic mice compared to littermate controls (Fig 2B) through quantitative PCR as previously mentioned. Reduced levels of Kmt2a, a writer of H3K4me $3^{48}$, are likely to decrease global H3K4me3 as it has been shown in previous studies ${ }^{46,49}$. Therefore, we decided to assess the functional influence of reduced Kmt2a on H3K4me3 levels. Of note, H3K4me3 is enriched globally at promoters of transcriptionally active genes ${ }^{50,51}$ and reduced H3K4me3 depositions at genes promoter negatively affect genes transcription ${ }^{52}$. We hypothesized that there could be a causal relationship between the downregulation of neuronal enriched genes and H3K4me3 marks at their promoters. To test this, we devised immunoprecipitation for $\mathrm{H} 3 \mathrm{~K} 4 \mathrm{me} 3$ from sorted neuronal nuclei and performed high throughput Chip sequencing as outlined in Fig 2B. We investigated the level of $\mathrm{H} 3 \mathrm{~K} 4 \mathrm{me} 3$ at the promoter of down-regulated genes by aligning Chip-seq reads to transcription start sites (TSSs) of those genes. Since lengths of gene promoters vary in genome $\mathrm{e}^{53}$, we examined $2 \mathrm{~kb}$ up- and downstream of transcription start site (TSS) as a proxy of gene promoters. In wild type mice for the down-regulated genes, we observed that the level of $\mathrm{H} 3 \mathrm{~K} 4 \mathrm{me} 3$ peaks slightly downstream of TSSs and gradually declines over the first $1 \mathrm{~kb}$ (Fig 3C). Previous studies have found a similar 
pattern of $\mathrm{H} 3 \mathrm{~K} 4 \mathrm{me} 3$ at $\mathrm{TSS}^{50}$. Interestingly, down-regulated genes have substantially reduced $\mathrm{H} 3 \mathrm{~K} 4 \mathrm{me} 3$ level at their promoters (TSS $\pm 2 \mathrm{~kb}$ (Fig 3C) in transgenic mice compared to those from littermate controls, suggesting a potential role of $\mathrm{H} 3 \mathrm{k} 4 \mathrm{me} 3$ in dampening gene expression.

In further comprehensive analyses, a total of $138026 \mathrm{H} 3 \mathrm{~K} 4 \mathrm{me} 3$ narrow peaks were detected in both groups, of which 8506 peaks were up-regulated while 7741 peaks were down-regulated in transgenic mice compared to littermate controls (Fig 3D). Significantly deregulated peaks are visualized in Fig 3D. 25\% of these deregulated peaks were annotated to be at the promoters $(\mathrm{TSS} \pm 2 \mathrm{~kb})$ of genes, while most of the other peaks fall into distal intergenic regions and other non-coding regions (Fig 3E). Genes with $\mathrm{H} 3 \mathrm{~K} 4 \mathrm{me} 3$ peaks are likely to be involved in RNA metabolism and protein-protein interaction at synapses (Fig 3F). Next, we studied genes characterized by (i) $\mathrm{H} 3 \mathrm{~K} 4 \mathrm{me} 3$ peaks at the promoters (TSS $\pm 2 \mathrm{~kb}$ ) and (ii) showed significant transcriptional deregulation (FDR $<0.05)$ in transgenic mice compared to controls. We thus limited our further analyses to 2293 genes that met these criteria and pairwise correlation analysis between RNA-seq and Chip-seq profile for these genes from the same set of samples was subsequently performed. 




Fig 3. H3K4me3 profile in transgenic and control mice

A) Hypergeometric overlap test among deregulated genes from this study, and altered genes from studies investigating Kat2a, Kmt2a, and Kmt2b knockouts in mice. Down-regulated genes from this study show considerably higher overlap with down-regulated genes from Kat2a and Kmt2a knockout mice B) Scheme of FACS from the mouse hippocampus (CA1 + CA3). C) NGS plot for down-regulated genes showing H3K4me3 peaks sharply declined at the promoter (TSS $\pm 2 \mathrm{~kb}$ ) of the down-regulated genes. D) Heatmap showing number of deregulated peaks (FDR < 0.05) and MA plot showing the distribution of up- and down-regulated peaks in transgenic mice compared to controls. $\mathrm{X}$ axis represents mean peak intensities (log2 scale) and Y axis shows fold change (log2). E) Pie-chart showing annotated peaks across different regions in the mouse genome (mm10) F) Annotated genes of deregulated peaks could contribute to RNA metabolism and protein-protein interactions at synapses. G) Left plot showing correlation between H3K4me3 peaks and RNA-seq expression for significantly deregulated genes. Based on correlation two clusters are presented. Correlation patterns of neuronal and glial cell 
type enriched clusters are represented as thick red line, dashed blue line respectively. Gene expression (middle panel) for corresponding genes are plotted for both control and transgenic groups. Color bars represent annotation for correlation co-efficient, RNA expression, and genotypes. H) Bar plot showing gene ontology of three clusters. $\mathrm{X}$-axis represents FDR (- $\log 10$ scaled) while $\mathrm{Y}$-axis represents the gene ontology biological processes.

We found two significantly correlated clusters based on this analysis (Fig 3G, Supplementary Table 4). One cluster representing down-regulated genes in transgenic mice had a positive correlation with the H3K4me3 profile. Since we initially observed down-regulated genes highly overlap with neuronal genes, we named this cluster as neuron-enriched cluster. Of note, we initially observed reduced $\mathrm{H} 3 \mathrm{~K} 4 \mathrm{me} 3$ globally for down-regulated genes (Fig 3C). Therefore, the downregulation of neuron enriched cluster and its positive correlation with down-regulated $\mathrm{H} 3 \mathrm{~K} 4 \mathrm{me} 3$ marks at their promoter further confirms a causal gene-epigenetic relationship at single-gene resolution (Fig 3G). The other cluster instead showed a positive correlation between RNA-seq and $\mathrm{H} 3 \mathrm{~K} 4 \mathrm{me} 3$. Gene sets of this cluster showed up-regulation in transgenic mice and thus was named glia enriched cluster. The negative correlation between gene expression and $\mathrm{H} 3 \mathrm{~K} 4 \mathrm{me} 3$ peaks at their promoter suggesting that other $\mathrm{kmt} 2 \mathrm{a}$ independent epigenetic modification and/or non-histone transcription factors could mediate the overexpression of this cluster. Given that we investigated the $\mathrm{H} 3 \mathrm{~K} 4 \mathrm{me} 3$ profile in the neurons, the upregulation of the gene cluster representing glial cell-type could be due to other possible epigenetic modifications.

Gene ontology analysis revealed that neuron-enriched cluster is likely to be involved in RNA splicing and protein folding (Fig 3H, Supplementary Table 4). Moreover, neuron enriched cluster gene sets are likely to be also involved in the post-synaptic organization and dendritic spines density (Supplementary Table 4). Therefore, H3K4me3 dependent reduction of these genes' expression may negatively affect synaptic plasticity, leading to poor cognitive performance in transgenic mice. In support of this hypothesis, the previous study showed that loss of $\mathrm{Kmt} 2 \mathrm{a}$ results in decrease of synaptic plasticity-related genes, an outcome that has been linked to observed cognitive deficits and attributed to a global decline in H3K4me3 profile $\mathrm{e}^{46}$. Glia enriched cluster is likely to be involved in increased autophagy, ER and oxidative stress response, and negative regulation of neuronal functions (Fig 3H, Supplementary Table 4). In summary, both $\mathrm{H} 3 \mathrm{~K} 4 \mathrm{me} 3$ linked reduction in RNA splicing and synaptic plasticity, as well as H3K4me3 independent exacerbation of ER/oxidative stress responses conjointly may have precipitated cognitive deficits in our heart failure animal model. 


\section{SAHA rescues early age cognitive deficits in CamkIISc transgenic mice}

Despite early cognitive deficits in transgenic mice, we reasoned that early therapeutic interventions might ameliorate memory impairments. In this study, we have found evidence that reduction of $\mathrm{H} 3 \mathrm{~K} 4 \mathrm{me} 3$ at the promoters of neuronal enriched and cognition related genes could be the potential underlying mechanism of cognitive deficits in the heart failure animal model. Accordingly, we asked whether we could restitute memory in these mice, through genome-wide increased distribution of $\mathrm{H} 3 \mathrm{~K} 4 \mathrm{me} 3$. We envisioned to accomplish this through histone deacetylase inhibitors. Histone deacetylase (HDAC) inhibitors are "chromatin openers" that favors gene expression and have been linked with memory improvement in cognitively impaired animal models ${ }^{42,54,55}$. Previous studies have shown that HDAC inhibitors could increase both histone actelylation and methylation levels ${ }^{56,57}$. We reasoned that Vorinostat (SAHA) could be a right HDAC inhibitor choice due to its effect of restoring the transcriptomic profile and potentially rescuing cognitive deficits in memory-impaired mice ${ }^{42}$. As the first step towards SAHA mediated interventions, we investigated SAHA's potential to increase H3K4me3 in neurons. Western blots revealed that SAHA not only increased H3K9ac intensity as expected, but also substantially increased H3K4me3 intensity after 1 hour of treatment in primary neuronal culture (Fig S3A). Since H3K9ac and H3K4me3 marks are similar in terms of genome-wide distribution and functionally related ${ }^{52,58}$, such an observation was of no surprise. Moreover, SAHA's potential to increase both H3K9ac and H3K4me3 level has been reported in previous studies, albeit in different cell types ${ }^{56,57}$. Concordantly, Chip-seq based analysis revealed that SAHA could induce genome-wide changes in both H3K4me3 and H3K9ac profile ${ }^{57}$.

Increased histone modifications and induction of a more relaxed chromatin state following SAHA treatment are likely to alter gene expression pattern. Therefore, we decided to investigate SAHA's potential to change gene expression patterns as an output of these epigenetic changes. In our previous study, we showed that cognitively impaired old mice display substantial changes in gene expression compared to cognitively healthy young mice ${ }^{42}$. We have observed similar gene expression changes in CamkII $\delta \mathrm{c}$ transgenic mice compared to controls (both up- and down-regulated genes) as shown in Fig S3B with a highly significant overlap between two data (see also Fig $1 \mathrm{~N}$ ). Benito et $\mathrm{al}^{42}$ showed that oral treatment of SAHA 
in old mice could not only induce a transcriptional profile similar to that of young mice but also lead to cognitive improvement. Comparative analysis with data from our heart failure model and previous study ${ }^{42}$ revealed that up- and down-regulated genes from CamkII $\delta$ c mice significantly overlapped with down- and up-regulated genes respectively in old SAHA vs. Old vehicle $^{42}$, highlighting SAHA's potential to target similar gene sets and to reverse their expression patterns in CamkII $\delta$ c transgenic mice (Fig S3B).

Both of these observations prompted us to examine the effects of SAHA directly on cognitive deficits. Therefore, transgenic mice were given Vorinostat (SAHA) $(0.67 \mathrm{~g} / \mathrm{L})$ in drinking water for 1 month from two months of age (TS). Another group of transgenic mice (TV) and additional littermate controls (WV) were given only vehicle in drinking water for an equal duration (Fig 4A). Previous studies reported that SAHA could cross blood-brain barrier ${ }^{59-62}$, have no negative influence on liver, and show limited effects on transcriptomics of cognitively healthy mice at the given dose ${ }^{42}$. After 1 month of treatment at the age of 3 months, mice were subjected to a similar battery of behavioral tests as previously mentioned. In the open field test, mice from all groups spent a similar time in the middle region of the field, traveled similar paths and displayed similar speeds (Fig 4B). These results suggest that there was no base level difference in terms of anxiety among groups as it was observed previously (see Fig 2). Next, we performed Barnes Maze to evaluate spatial learning and memory retrieval in mice. Transgenic mice with vehicle (TV) exhibited higher escape latencies to find the escape hole compared to littermate controls (WV), akin to previous observations (see Fig 2). However, transgenic mice treated with SAHA (TS) performed similarly to littermate controls (WV) (Fig 4C). Increased escape latency in TV may be attributed to a decline 

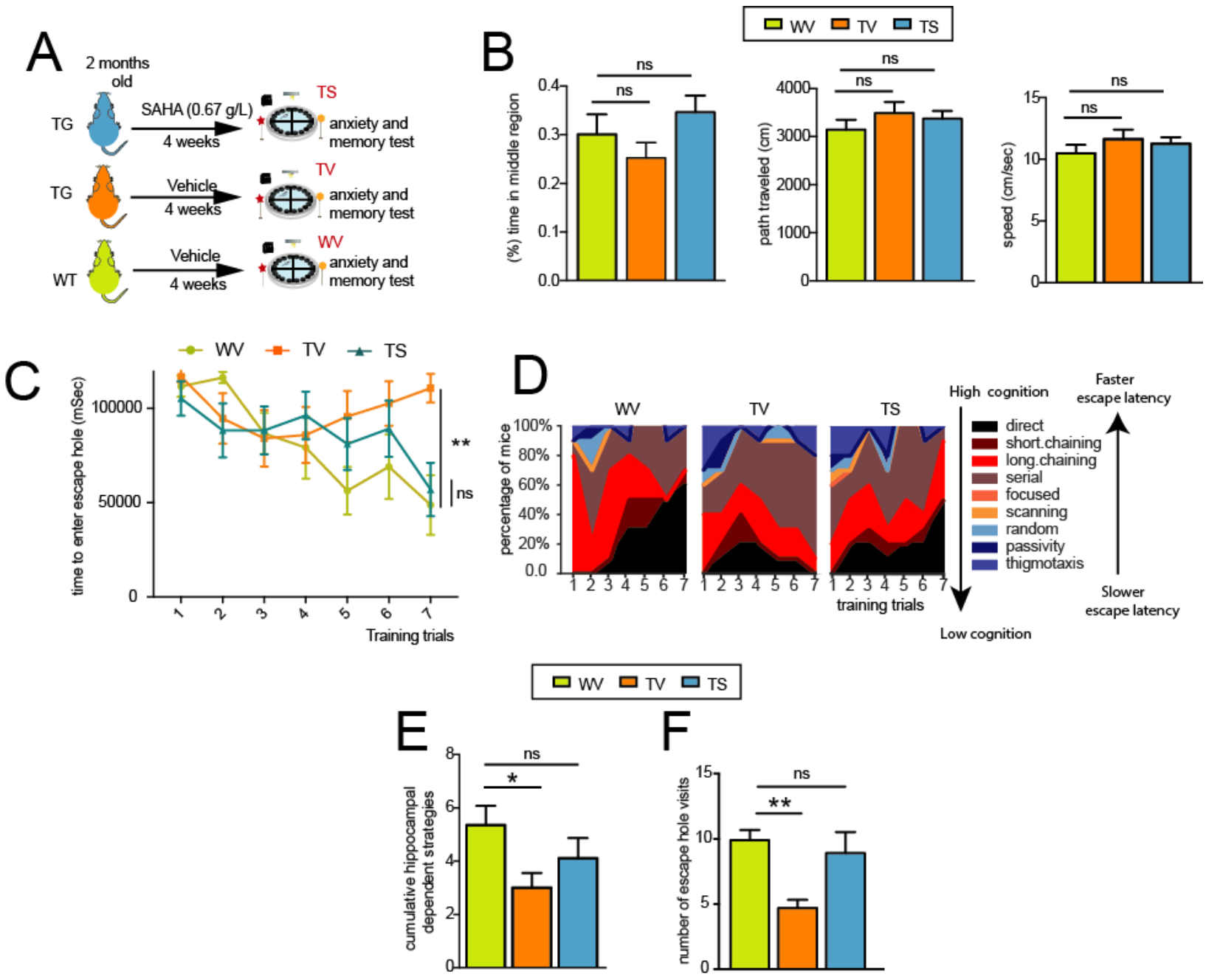

Fig 4. A behavioral experiment in SAHA/vehicle treated mice

A) A schematic outline showing the experimental settings. One group of transgenic mice was given SAHA (TS) while other group was given vehicle (TV) and their behavioral performance were compared to that of control mice treated with vehicle (WV). Drug/vehicle treatment was conducted for 4 weeks ( $\mathrm{n}=10$ each group). B) Percentage of time in middle regions (left panel), path traveled (middle panel), and speed (right panel) are shown from open field test (one-way ANOVA). C. Escape latency to target hole in three different groups over seven training trials (Two-way ANOVA, $* * \mathrm{p}<0.01$ ). D) Area curve plot for different strategies that mice employed during trials. Colors represent strategy. E) cumulative hippocampal-dependent strategy scores from training trials and F) number of target visits during probe test among groups (One-way ANOVA, ${ }^{*} \mathrm{p}<0.05,{ }^{* *} \mathrm{p}<0.01$ ). Error bars indicate mean \pm sem.

in direct, short and long chaining strategies, particularly at the $7^{\text {th }}$ trial (Fig 4D). Consistently, a cumulative score calculated based on these strategies (see methods for details) showed significant cognitive performance decline in TV mice across trials compared to WV mice, while in TS group the learning ability was partially rescued (Fig 4E). A memory recall test during the probe test confirmed our previous observation (Fig 2F) of substantial memory decline in transgenic mice. However, TS mice displayed memory reinstatement due to SAHA 
treatment (Fig 4F). Based on these data, we conclude that oral treatment of SAHA in transgenic mice can ameliorate cognitive deficits (Fig 4).

\section{SAHA may restore transcriptomic changes in transgenic mice}

Based on our previous analysis (Fig S3B), we hypothesized that SAHA mediated cognitive rescue as observed in transgenic mice could be due to the reinstatement of the brain transcriptomic profile in transgenic mice. Therefore, we investigated changes in gene expression as a proxy of potential genome-wide increased distribution of $\mathrm{H} 3 \mathrm{~K} 4 \mathrm{me} 3$ mediated by SAHA. To this end, we sacrificed mice and micro-dissected CA1 region from all the three experimental groups; wild type vehicle (WV), transgenic vehicle (TV) and transgenic SAHA (TS). RNA was then isolated, and RNA-seq was performed (Fig 5A). Phenotyping of these mice showed that body weight and lung weight were not affected in any of the experimental groups (Fig S5B-C). Similar results were observed for the whole heart and left ventricle (Fig S5D, Fig S5E). After adjusting for both technical and biological covariates, our RNA-seq data analysis revealed major deregulation of mRNAs in the transgenic vehicle group compared to littermate controls (Fig S4A). This result supports previous observation (Fig 1J), and this consistency was further reflected in the functional analysis performed using deregulated genes (Fig 5B). RNA splicing and protein folding were down-regulated while increased unfolded protein response, oxidative and ER stress response, autophagy and neuronal death were observed in TV mice. Interestingly, SAHA could partially reinstate gene expression changes in TS mice (Fig S4B), an effect which was observed for both up (38\%) and down-regulated (39\%) genes (Fig S4B). Concordantly, SAHA could reverse deregulated concerned biological processes in transgenic mice (Fig $5 \mathrm{~B}$ ) and was likely to increase non-coding RNA mediated regulations (Fig 5B). We wanted to test whether SAHA could rectify splicing in TS group and reasoned that differential exon usage would be a proxy to different splicing events, and informing us of any bias to use one exon over another. Therefore, we performed differential exon usage in TV and TS, both as compared to WV. SAHA led to a dramatic increase of differential exon usage in TS vs. WV condition compared to TV vs. WV (Fig S4C). In support of this, similar SAHA mediated increased differential exon usage has been illustrated in a previous study ${ }^{42}$ 
To gain better insights about molecular mechanisms that underpin this SAHA mediated cognitive rescue, we further investigated the RNA-seq data in greater details and asked how the expression of different co-expressed clusters would change among groups. Recent studies showed that co-expression of regulatory clusters could point to key mechanisms underlying disease conditions ${ }^{63-66}$. We aimed to find hub genes of the interesting clusters and hypothesized that these hub genes could be under the regulation of other epigenetic mechanisms e.g., through microRNAs (Fig 5C). The above hypothesis was based on the previous observation that, SAHA could increase non-coding RNA regulations (Fig 5B). To this end, we performed Weighted Gene Co-expression Analysis ${ }^{67}$ and found 14 different clusters (Fig 5D-E, Fig S4E). We limited our analysis to modules that show differential expression among groups and found two such modules. The first module, RNA module 1 module, displayed reduced expression in TV (Fig 5D) compared to WV but partial rescue in TS group (Fig 5D). Gene ontology analysis indicated that the gene members of RNA module 1 are likely to be involved in action potential, cognition, and learning memory (Fig 5E, Supplementary Table 5). Therefore, RNA module 1 cluster represents a gene cluster linked to cognition. Regulatory network of this cluster is illustrated by hub genes (Fig 5E) and evidence that most of these genes are key players in context of learning and memory are summarized in Supplementary Table 6. Downregulation of these memory-related hub genes in TV group may contribute to cognitive decline and SAHA's potential to modulate their expression levels make it an ideal candidate for therapeutic interventions in memory disorders. In contrast, expression of the second module, RNA module 2 module, increased in transgenic vehicle (TV) group compared to littermate controls (WV) (Fig 5F), but partially normalized in transgenic + SAHA group (TS) (Fig 5F). RNA module 2 cluster member genes significantly overlap with oxidative stress response, autophagy and different stress-induced kinase response biological processes (Fig 5G, Supplementary Table 5). Since our gene ontology analyses consistently point towards increased oxidative response, ER stress, and autophagy in addition to reduced cognition related processes, we decided to investigate the effects of these different stress conditions on gene expression. We examined whether hypoxic and ER stress conditions could induce similar gene expression changes as observed in heart failure mice. To this end, first we compared the deregulated genes in transgenic vehicle (TV) group with genes deregulated in hypoxic condition from a recent study ${ }^{68}$. Pasca et al. ${ }^{68}$ showed that hypoxia induces distinctive expression changes in neurons after 24 and 48 hours. 

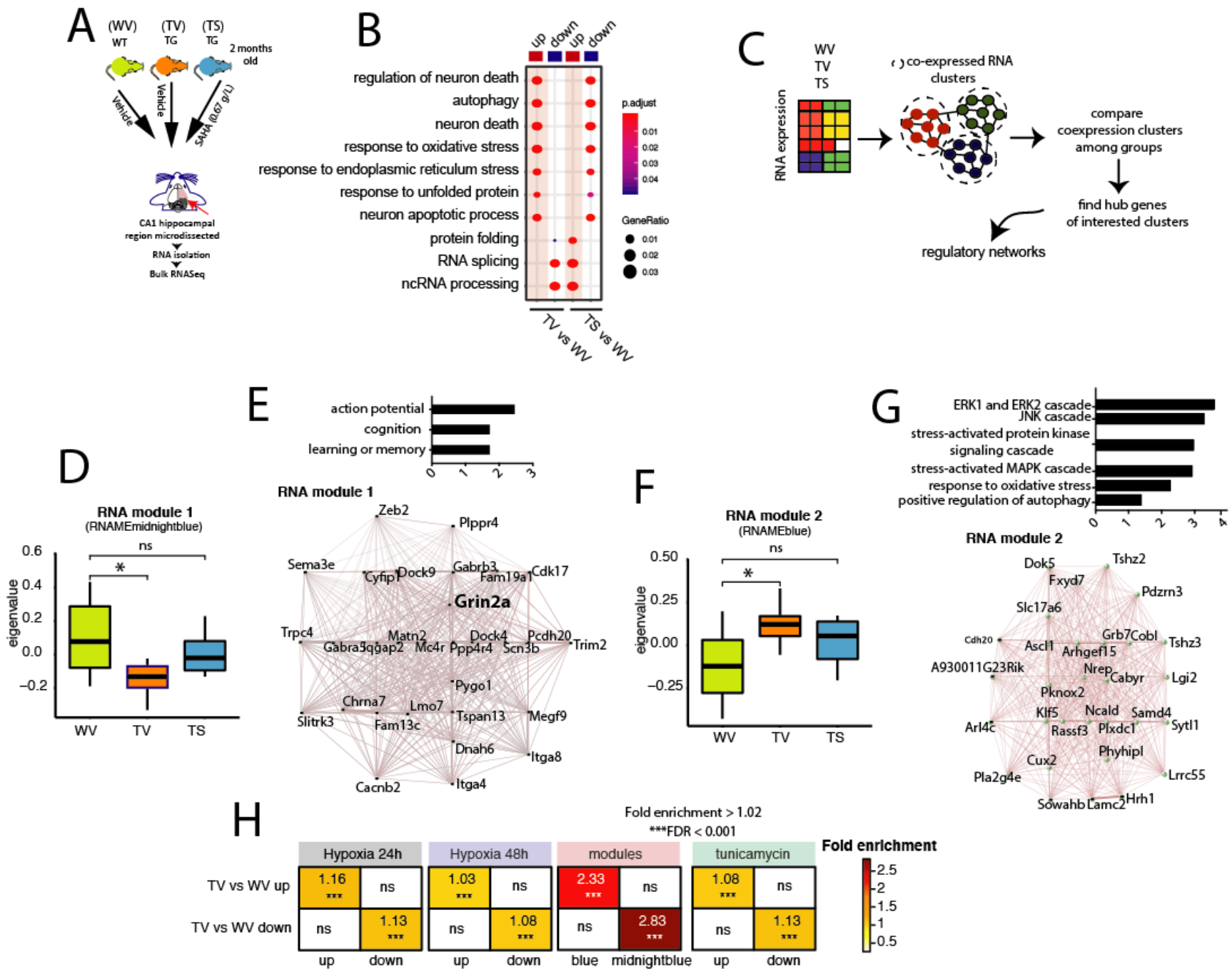

Fig 5. Orally given SAHA rescues cognitive performance through possible combinatorial effects.

A) Scheme of RNA-seq from studied experimental groups $(n=10$ each group). $\mathrm{TS}=$ Transgenic mice + SAHA; $\mathrm{TV}=$ transgenic mice + vehicle; $\mathrm{WV}=$ control mice + vehicle. B) Featured biological processes from gene ontology analysis of the deregulated genes is summarized in dot-plot. C) Outline of a gene co-expression-based workflow to investigate underlying molecular mechanisms. D) Expression of RNA module 1 among the three groups. ${ }^{*} p<0.05$, Kruskal Wallis test E) (top panel) Gene ontology analysis of gene members from RNA module 1. (bottom panel) Network representing top 30 hub genes of the gene network based on RNA module 1. Gene network was visualized in VisANT 5.0. F) RNA module 2 and its expression among three groups ${ }^{*} \mathrm{p}<0.05$, Kruskal Wallis test G) (top panel) functional annotations of genes from RNA module 2. (bottom panel) top hub genes $(n=30)$ in gene correlation network of RNA module 2. H) Heatmap summarizing results from hypergeometric tests for deregulated genes in transgenic mice with hypoxic and tunicamycin induced ER stress conditions. Up and down-regulated genes $(\mathrm{FDR}<0.05)$ were determined by comparing with corresponding controls. Fold enrichment cutoff: 1.2 and enrichment significance cutoff: FDR $<0.001$. Color intensity represents fold enrichment.

We found that the up-regulated genes in our transgenic vehicle (TV) group overlap significantly with those of 24-hour and 48-hour long hypoxic treatment (Fig 5G). Moreover, up-regulated genes significantly overlap with RNA module 2 modules as well (Fig 5F, 5G), confirming that these changes are stress responsive. Next, we modeled ER stress in a primary neuronal culture system (Fig S5A) through a 6-hour long tunicamycin treatment. Induction of 
ER stress using tunicamycin has been described elsewhere ${ }^{69-72}$. The genes up-regulated after tunicamycin treatment compared to vehicle only controls showed significant overlap with upregulated genes in transgenic mice treated with vehicle (TV) compared to littermate controls (WV). In summary, gene expression changes in our transgenic mice overlap with those from increased hypoxic and ER stress responses. So far, biological process analysis revealed that SAHA is likely to reduce these stress responses (Fig 5B, 5F). This was confirmed in our cell culture model, as SAHA pretreated neurons showed a weaker ER stress response compared to neurons treated with only tunicamycin (Fig S5B). Further confirmation came from qPCR experiments performed for selected ER stress responsive genes in mice. Increased expression of ER stress responsive genes (e.g. Bcap31, Fez2) were noted in TV mice, but their expression was normalized in transgenic mice treated with SAHA (TS) (Fig S5C). Therefore, data from cells and animal model show that SAHA can restore transcriptomic homeostasis and have protective role against oxidative and endoplasmic stress responses.

\section{microRNAs may play a critical role in compensation of stress response}

This observation led us to ask our next question of how SAHA, a histone deacetylase inhibitor, that generally opens up chromatin and facilitates increased gene expressions could reduce expression of stress-related genes. To explain this seemingly somewhat inverse relation between SAHA and gene expression, we reasoned that SAHA driven increased microRNA expression might play a key role in regulating in ER and oxidative stress responses.

To investigate this hypothesis, we carried out small RNA sequencing from TV, WV, and TS groups (Fig 6A). Similar to RNA-seq, we performed weighted co-expression analysis on all samples (Fig 6B). Our analysis revealed five different clusters based on co-expression patterns (Fig 6C, Fig S6D) and we evaluated their expression changes among groups using eigenvalue. We limited our analysis to two clusters that displayed differential expression among groups (Supplementary Table 7). Expression of the first cluster, microRNA module 1, was notably up-regulated in TV but fully rescued in TS (Fig 6C). In contrast, microRNA module 2 showed reduced expression in the TV group. Interestingly, oral administration of SAHA could also fully compensate this change in TS group (Fig 6D). Since microRNAs are known for silencing gene expression, we hypothesized that the increased expression of microRNA module 2 in 
transgenic mice following SAHA treatment (compared to transgenic mice with vehicle) might negatively affect mRNAs involved in stress responses. If this hypothesis holds true, we expected a negative correlation pattern between increased microRNA and decreased mRNA expression. To investigate microRNA-mRNA interactions between TS and TV, we performed a pairwise correlation taking normalized counts of

differentially deregulated microRNAs and genes from TS vs. TV comparison. Our analysis revealed both positive and negative interactions and full results from this analysis is summarized in Supplementary Table 8. Interestingly, we found a subset of microRNAs from microRNA module 2 that show negative correlations (Fig 6E) in expression with hub genes from RNA module 2 module, the gene cluster that is linked to stress responses and autophagy (Fig 5F). Next, we asked whether the expression of this subset of microRNAs from microRNA module 2 could be under epigenetic regulation. Therefore, we retrieved their promoters and limited our analysis to those microRNAs whose expression is dependent on the host gene promoter. Mapping of $\mathrm{H} 3 \mathrm{~K} 4 \mathrm{me} 3$ peaks on the promoter of these host genes revealed that reduction in the expression of this subset of microRNAs in TV was due to reduced $\mathrm{H} 3 \mathrm{~K} 4 \mathrm{me} 3$ levels at promoters, at least in part, of their host genes (Fig S6E). These data, coupled with observation of TS mice, demonstrate that microRNAs may contribute to cognitive improvement by silencing stress-responsive genes. 
A

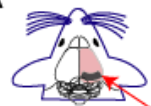

CA1 hippocampal region microdissected

$$
\text { RNA isolation }
$$$$
\text { Small RNAseq }
$$

B

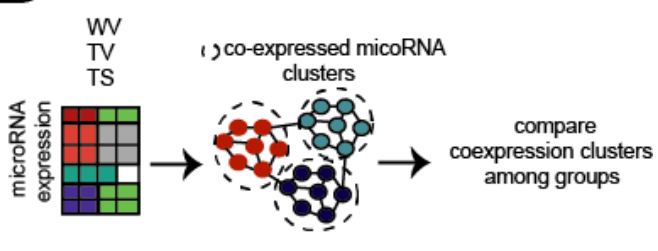

\section{wV $\mathrm{WV}$ TS}


Fig 6. microRNA may reduce the expression of $E R$ responsive genes

A) Scheme of sample preparation and sequencing for genome-wide microRNA analysis. B) Scheme of coexpression analysis using all expressed microRNAs from all experimental groups (n, WV:9, TV:7, TS:9) C-D) Two significantly deregulated clusters namely microRNA module 1 (C) and microRNA module 2 (D) are shown. Kruskal Wallis test. ${ }^{* * p}<0.01$. Box plot center, lower and upper hinges represent the median, first and third quartiles respectively E) Heatmap summarizes significant negative correlation (FDR $<0.05$ ) as observed between microRNA members in microRNA module 2 and hub genes from RNA module 2 (Fig 5E). 


\section{Discussion}

Evidence emerging from recent functional imaging data show that hypoperfusion due to chronic heart failure results in tissue loss of different brain regions including hippocampus ${ }^{8}$, the region that is inherently linked with spatial navigation, learning, and memory. However, how the brain is functionally affected at the molecular level due to the hypoperfusion remains poorly underexplored. To investigate this, in this study, we combined comprehensive systems biology using high throughput sequencing data and cognitive phenotyping along with in vitro as well as in vivo experiments.

Using a heart failure (HF) mouse model, we demonstrate that reduced cardiac function can contribute to substantial changes in hippocampal gene expressions. Our data show that increased expression of genes is related to stress response processes, including endoplasmic reticulum (ER) stress and autophagy. On other hand, down-regulated genes are likely to be involved in cognition and protein folding. In line with our data, recent study using single-cell RNA-seq data from Alzheimer's disease (AD) demonstrated that similar processes could be altered in early $\mathrm{AD}$ pathology ${ }^{73}$. Moreover, ER stress has been linked with different neurodegenerative diseases ${ }^{41}$. Concordantly our comparative analysis showed that transcriptomic changes due to heart failure share similar profile to that from other diseases where memory is substantially impaired (e.g., aging, AD, Frontotemporal dementia (FTLD) and Parkinson's disease (PD). Therefore, cardiac failure mice manifest conserved molecular changes associated with memory disorders. Thus, heart failure mice could be an excellent model to investigate brain's early molecular changes in the context of dementia. Since hypoperfusion precedes major brain structural changes and cognitive deficits, early molecular changes owing to hypoperfusion may be used as markers for early diagnosis and to evaluate the risk of dementia.

Our HF transgenic mice with reduced ejection fraction displayed both spatial learning and memory deficits (Fig 2) at an early age. This finding corroborates with a study on congestive cardiac failure model mice ${ }^{74}$, which exhibited memory deficits in the water maze. However, the previous study ${ }^{74}$ modeled myocardial infarction by ligation of left coronary artery and thus represent post-surgical sudden onset of heart failure. Nevertheless, both results confirm the association of cardiac failure with memory deficits. In agreement with this, the populationbased Rotterdam study on human HF patients showed that diastolic dysfunction in the heart 
was associated with an increased risk of dementia ${ }^{75}$. Two other parallel studies found similar results and reported a 1.8-2.1 fold increased risk of all dementia in HF patients ${ }^{76,77}$. Consistently, global cognitive decline affecting different domains including executive function, attention, episodic memory, learning and working memory has been reported in human HF patients ${ }^{78,79}$. However, detection of early cognitive changes in humans remains a challenge with currently available neuropsychological tests ${ }^{80-82}$. This could be partly explained by the fact that general cognitive tests to evaluate cognitive performance in human does not involve spatial orientation and navigation strategies. Recent evidence revealing that spatial deficits have higher specificity over other cognitive measures ${ }^{83}$ and have potential to determine early cognitive changes ${ }^{82}$. Newly developed virtual reality testing is showing promise of being easily applicable in clinical settings and more sensitive in identifying early spatial navigation deficits in human ${ }^{84,85}$. Furthermore, both human real-life version and computerized version of Morris water maze was able to find early topographical disorientations in patients ${ }^{86,87}$. Given that spatial navigation deficits is not observed in healthy older individuals ${ }^{88}$, this may allow earlier diagnosis of age-independent cognitive decline in HF patients. Moreover, analysis of spatial performance in human HF patients will allow better translation of our mouse HF data from the present study to humans.

Based on our comparative analysis, we hypothesized that cognitive deficits in HF mice could be attributed to the reduced level of Kmt2a, H3K4 methyltransferase. This reasoning was based on previous observations that mice with reduced level of $\mathrm{Kmt} 2 \mathrm{a}$ displayed deficits in spatial memory and long-term potentiation ${ }^{46,49,89}$. We previously linked H3K4me3 as a reliable indicator of the functionality of $\mathrm{Kmt} 2 \mathrm{a}^{46}$. In consistent to this, we observed a global decline of $\mathrm{H} 3 \mathrm{~K} 4 \mathrm{me} 3$ at promoter of cognition related genes in HF mice. Reduction of H3K4me3 was previously observed with reduced a level of $\mathrm{Kmt} 2 \mathrm{a}^{46,90}$. Since H3K4me3 has been linked with maintenance of memory ${ }^{90,91}$, thereby, decreased level of $\mathrm{H} 3 \mathrm{~K} 4 \mathrm{me} 3$ leading to downregulation of cognition related genes may underpin cognitive deficits in HF mice. Interestingly, expression of stress-responsive genes was up-regulated despite the reduction of $\mathrm{H} 3 \mathrm{~K} 4 \mathrm{me} 3$ level at their promoters. This may be due to their expression being regulated through nonhistone transcription factors or other histone modifications. Moreover, the disparity may come from the fact that we performed $\mathrm{H} 3 \mathrm{~K} 4 \mathrm{me} 3$ from neurons, whereas these genes represent mixed cell types (Fig 1). 


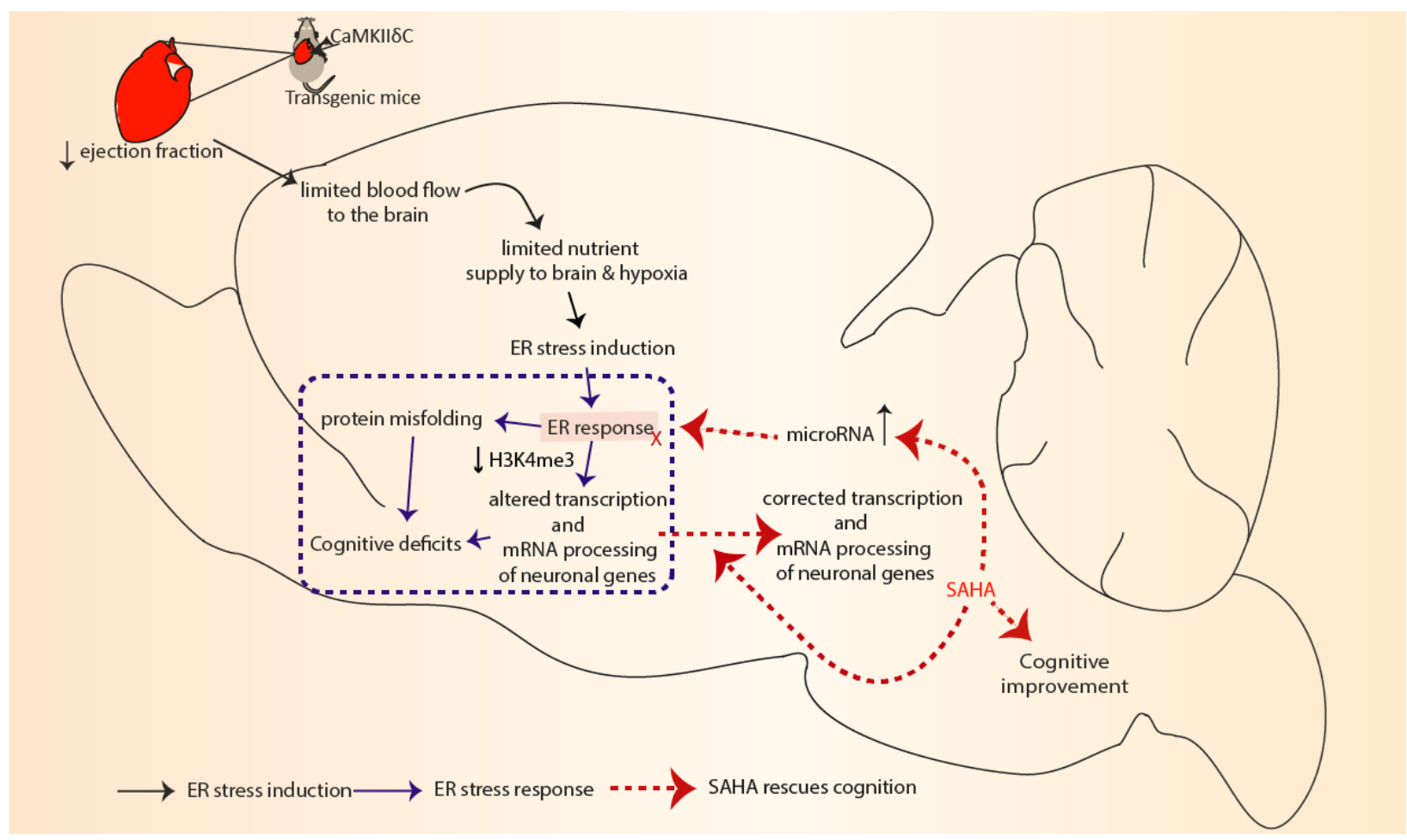

Fig 7. SAHA mediated cognitive rescue in mice following heart failure.

Heart failure (HF) is characterized by reduced blood flow (hypoperfusion) that can lead to limited nutrient and oxygen supply to the brain. Nutrient deprivation and hypoxia are likely to positively influence endoplasmic reticulum (ER) stress in the brain. Increased ER stress, in turn, may induce epigenetic and transcriptomic changes in the brain, including downregulation of memory-related genes. This study points out of the reduced level of $\mathrm{H} 3 \mathrm{~K} 4 \mathrm{me} 3$ as an underlying molecular mechanism for the down-regulation of memory-related genes following hypoperfusion. We report increased protein misfolding in the HF brain that can be attributed to the increased ER stress response. Collectively, these molecular changes can contribute to cognitive deficits after HF. Vorinostat (SAHA), an inhibitor of histone deacetylase (HDAC), can restore aberrant gene expression changes for the memory-related genes and thereby exerts positive benefits to cognition. Moreover, SAHA can partly restitute microRNAome, that in turn, can counteract stress responses.

Available drugs that improve cardiac failure pathology have a limited effect on cognitive pathology and dementia risk in HF patients ${ }^{15,16,19-21}$. Based on our observation that HF leads to brain-related epigenetic changes of gene expression that resemble changes seen in neurodegenerative diseases, we tested an epigenetic drug. Vorinostat (SAHA) is an FDA approved drug that acts as an inhibitor of histone deacetylase and thereby promotes gene expression by opening the chromatin structure. Its efficacy is currently being tested in human clinical trial for dementia (ClinicalTrials.gov Identifier: NCT03056495). We report that hippocampal-dependent spatial learning and memory as observed in mice with failing heart could be rescued with 4 weeks long oral administration of SAHA. Our findings are in line with previous study showing oral treatment of SAHA could ameliorate cognitive deficits in aged and Alzheimer's mouse model ${ }^{42}$. Moreover, the same study reported no detrimental effect of 
SAHA on peripheral organs ${ }^{42}$ and others have shown that it can cross blood-brain barrier ${ }^{60,62}$. Consistently, we failed to note significant changes in cardiac phenotype, particularly cardiac hypertrophy, after SAHA treatment. Interestingly, this finding is in sharp contrast to a previous study $^{92}$ showing that TSA, another HDAC inhibitor, could ameliorate cardiac hypertrophy. However, in support of our observation, another study found no effect of SAHA on heart function ${ }^{93}$. Therefore, the functional impact of different HDAC inhibitors on the heart may vary.

Our results show that SAHA could partially restore the hippocampal transcriptomic profile in HF mice and that was reflected in the reinstatement of biological processes as well (Fig 7). Interestingly, SAHA specifically increased the expression of a cognition related gene expression cluster. Hub genes of this cluster include Zeb2, Plppr4, Sema3e, Dock9, CYFIP1, GABRb3, Grin2a, Trpc4, Gabra5, Iqgap2, Matn2, Mc4r, Dock4, Scn3b, Pcdh20, Tim2, Slitrk3, Chrna7, Itga8, Cacnb2, Itga4; all of which have all been previously linked to synaptic plasticity, axonal outgrowth, neuronal activity, dendritic development and memory ${ }^{94-114}$. Additionally, previous studies showed that mice with reduced expression of GABRb3 ${ }^{99}$, Gabra5 $5^{102}$, Trpc $4^{101,115}$ CYFIP $1^{115}$, Chrna $7^{111,116}$ genes developed memory deficits while Mc4r ${ }^{105}$ gene activation rescued cognitive deficits. Therefore, by suppressing the expression of these critical genes, cardiac failure induced hypoperfusion may contribute to cognitive decline. In contrast, SAHA's potential to increase their expression make it an ideal candidate for therapeutic interventions to improve memory after heart failure. Moreover, hub genes related to cognition also include novel gene sets (Lmo7, Pygo1, Fma13c, Tspan13, Cdk17) that have not yet been investigated in the context of learning and memory. Functional experiments on these genes in the future may shed more light on pathomechanisms during the cognitive decline in HF. Furthermore, we observed partial down-regulation of different stress-responsive genes after SAHA treatment. We reproduced SAHA's protective role against ER stress in cell culture using RNA-seq data and later confirmed it in vivo through qPCR. In line with this, previous studies reported SAHA's positive role in maintenance of ER homeostasis ${ }^{117}$ and protection against stress responses ${ }^{118}$.

The small RNA-seq data further provided insight into the mechanism by which SAHA could lower the expression of stress-related genes. Our in-depth analysis revealed a microRNA cluster that was up-regulated in transgenic mice (TV) but normalized following SAHA (TS) treatment. miR-122-5p, miR-204-5p, miR-211-5p, miR-221-3p, miR-23a-3p, miR-708-5p are 
part of this cluster and have previously been associated with endoplasmic stress-responsive microRNAs ${ }^{119}$. Our study also provides a list of microRNAs from this cluster that may be important to understand stress responses on microRNAs in great detail (Supplementary Table 7). We also report a second cluster of microRNAs whose expression was down-regulated in TV but fully rescued in TS. We found that a subset of microRNA members from this cluster showed negative correlations in expression with stress-responsive hub genes. Moreover, decreased expression of subset microRNAs in TV could be explained, at least in part, by reduced H3K4me3 level at promoters of their host genes (Fig S6E). Therefore, SAHA mediated increased expression of these microRNAs may contribute to down-regulation of hub genes related to stress. In addition to the restoration of transcriptomic profile, SAHA's potential to rescue microRNA homeostasis underpins its effectiveness against cognitive deficits in the context of heart failure.

In conclusion, we provide evidence that HF leads to epigenetic changes in the hippocampus that can contribute to memory impairment. We suggest that epigenetic drug might be a suitable strategy to treat cognitive dysfunction in HF patients and lower their risk of developing dementia. While future clinical studies will be needed to test the efficacy of SAHA in humans, our study highlights its potential as a pharmacological intervention to ameliorate memory impairment following heart failure. 


\section{Methods \& Materials}

\section{Animals}

CamkII $\delta$ c transgenic mice were bred according to a previous study ${ }^{25} .2-3$ months of transgenic mice and corresponding littermate controls were used in this study. All animals were housed in standard cages on $12 \mathrm{~h} / 12 \mathrm{~h}$ light/dark cycle with food and water ad libitum. Experimental protocols were approved by FELASA. Animals were sacrificed by cervical dislocation. Hippocampal sub-region CA1, CA3 regions were isolated, snap frozen in liquid nitrogen and stored at $-80^{\circ} \mathrm{C}$. The hearts were dissected by a cut above the base of the aorta and perfused with $0.9 \%$ sodium chloride solution until blood free, snap frozen in liquid nitrogen and stored at $-80^{\circ} \mathrm{C}$. In addition, lung and tibia were extracted and their respective weight or length were determined.

\section{Echocardiography}

The heart function and dimensions were examined by echocardiography using a Vevo 2100 imaging platform (Visualsonics) with $30 \mathrm{MHz}$ transducer (MS-400). The animals were anesthetized with isoflurane (1-2\%) and M-mode sequences of the beating heart recorded in the short-axis and the long axis, respectively. The images were used to determine the left ventricular end-diastolic and end-systolic Volumes (area*length*5/6). These parameters were used to calculate the ejection fraction as indicator of left ventricular heart function. The investigator was blinded to genotype and age.

\section{Histology}

Heart was fixed in $4 \%$ paraformaldehyde for 24 hours at $4^{\circ} \mathrm{C}$, dehydrated in an ethanol series, and embedded in paraffin for sectioning. The $5 \mu \mathrm{m}$ paraffin sections were post-fixed with Bouin's solution (HT10132, Sigma Aldrich) and stained using Masson's trichrome staining kit (Sigma, HT15-1KT) according to the manufacturer's instructions. Bright field images were acquired with the BX43 light microscope (Olympus). 


\section{Behaviourial tests and data analysis}

\section{Open Field \& Barnes Maze}

Open field test was performed according to Bahari-Javan et al ${ }^{120}$. Briefly, mouse was placed gently in the middle quadrant of an open field and let it explore for 5 minutes. The travel trajectories were recorded using VideoMot (TSE-Systems). Barnes Maze experiment was performed according to Sunyer et al ${ }^{121}$ with slight modifications. Details are summarized in Supplementary File.

\section{RNA isolation and sequencing}

RNA isolation was performed using RNA Clean and Concentrator kit according to manufacturer protocol without modifications. Concentration was measured on nanodrop and quality of RNA was evaluated. For mRNA sequencing, $500 \mathrm{ng}$ total RNA was used as input to prepare cDNA libraries according to Illumina Truseq and $50 \mathrm{bp}$ sequencing reads were run in HiSeq 2000. For small RNA sequencing, $100 \mathrm{ng}$ total RNA was used as initial input. Small RNA was enriched using size selection from based on gel. cDNA library and sequencing have been performed according to manufacturer's protocol (NEBNext Small RNA library prep set for Illumina). Next generation sequencing was performed on HiSeq 2000 platform.

\section{Chromatin immunoprecipitation for $\mathrm{H} 3 \mathrm{~K} 4 \mathrm{me} 3$}

Chromatin immunoprecipitation was performed according to (Halder et al., 2016) with $0.2 \mu \mathrm{g}$ chromatin and $1 \mu \mathrm{g} \mathrm{H} 3 \mathrm{~K} 4 \mathrm{me} 3$ (ab8580) antibody. ChIPseq library preparation was performed using NEBNext Ultra II DNA library preparation according to manufacturer's protocol. 2nM libraries were pooled and sequenced in Illumina Hiseq 2000 with 50-bp single end reads. Details are given in Supplementary File.

\section{Oral administration of SAHA}

SAHA was dissolved in drinking water according to a previous study ${ }^{42} .0 .67 \mathrm{~g}$ of SAHA (Cayman Chemical Co.) was dissolved in 11 of drinking water containing $18 \mathrm{~g}$ of $\beta$ cyclodextrin (Sigma-Aldrich, Ref 332607). The solution was heated at $70^{\circ} \mathrm{C}$ and mixed on a magnetic stirrer until SAHA was fully dissolved and left it overnight to cool down. The next day, Animals were given drug mixed water Control groups only received water with $\beta$ - 
cyclodextrin $(18 \mathrm{~g} / 1 \mathrm{~L})$. The previous study has showed that the given dose in the mouse would be equivalent to $375 \mathrm{mg} /$ day in humans which is within the tolerable dose.

\section{Modeling endoplasmic reticulum stress in primary neurons}

Primary neuronal culture was prepared according to Eva benito et al. ${ }^{122}$ (see Supplementary File for details). Experiments were performed at DIV 10. Endoplasmic reticulum stress was induced in primary hippocampal neurons using Tunicamycin (Sigma Aldrich). $2 \mathrm{ug} / \mathrm{mL}$ of Tunicamycin was added to primary neuronal culture and incubated for 6 hours and compared to those treated with DMSO for same time. For SAHA treatment, $1 \mu \mathrm{M}$ of vorinostat (SAHA, Cayman Chemical Co.) was added to culture 1 hour prior to Tunicamycin treatment. Neurons were treated with Trizol and stored at $-80^{\circ} \mathrm{c}$ until RNA isolation. RNA isolation was performed as discussed earlier.

\section{Quantitative RT-PCR}

qPCR (q-PCR) primers were designed using Universal probe library Assay Design Center and were purchased from Sigma. Transcriptor High Fidelity cDNA Synthesis Kit (Roche) was used to prepare cDNA. UPL probes were used for quantification and data was normalized to HPRT1 expression as internal control. Relative gene expression was analyzed by $2-\mathrm{ddCt}$ method. Primer sequences are summarized in Supplementary File.

\section{Western blot}

Chromatin-bound nuclear fractions were extracted from cultured cells using the Subcellular Protein Fractionation Kit for Cultured Cells (Thermo-Scientific, 78840). Western blot was performed according to previous study ${ }^{120}$. To quantify H3K4me3 and H3K9ac levels H3K4me3 (abcam, ab8580) antibody, H3K9ac (abcam, ab4441) antibody were used respectively. Unmodified H3 level measured with H3 antibody (abcam, ab1791) was used as internal control. Detailed procedure is summarized in Supplementary File.

\section{Bioinformatics analysis}

Bulk RNA Sequencing data analysis has been performed according to Wendeln et $\mathrm{al}^{32}$. Small RNA sequencing files were analyzed according to using miRDeep2. A wrapper of the steps applied during mapping and counting is uploaded and available as package 
(https://github.com/mdrezaulislam/MicroRNA). Differentially expressed genes or microRNAs were determined using mixed linear model accounting for technical and biological covariates. For linear mixded effects model, limma in $\mathrm{R}$ was implemented. Biological processes were analyzed using Gene Ontology (http://geneontology.org/). For pathway analysis KEGG (https://www.genome.jp/kegg/), Reactome (https://reactome.org/) databases were used. Exon counts were generated using DEXSeq (http://bioconductor.org/packages/DEXSeq/). Hypergeometric test analysis was performed using GeneOverlap (http://bioconductor.org/packages/GeneOverlap/). H3K4me3 narrow peaks were called using MACS2. Chip peaks were mapped at promoter (TSS $\pm 2 \mathrm{~kb}$ ) of genes using ngs.plot. Results from pairwise correlation between $\mathrm{H} 3 \mathrm{~K} 4 \mathrm{me} 3$ peaks and RNA expression was visualized using EnrichedHeatmap. Weighted co-expression analysis for both microRNAs and mRNAs was performed using WGCNA ${ }^{67}$. External gene expression datasets that have been used from other studies were downloaded from NCBI GEO (https://www.ncbi.nlm.nih.gov/geo/) and mapped in house to have consistency in results. Details of microRNA promoter and host genes and other analysis are summarized in Supplementary File.

\section{Statistical analysis}

All the statistical analyses as mentioned in the main text are performed in Prism (version 7.0) or in $\mathrm{R}$.

\section{Acknowledgements}

Authors thanks all lab members for their critical inputs to this work.

\section{Author contribution}

MRI coordinated, performed experiments \& analyzed data. MSS, RMH, DL, TB, MJM, SB, AK, JC performed experiments. EV, DMK provided technical supports. FS, KT, AF conceived the project. AF, MRI wrote manuscript.

\section{Competing Interests}

Authors declare there is no conflict of interest. 


\section{References}

1 Satizabal, C. L. et al. Incidence of dementia over three decades in the Framingham Heart Study. New England Journal of Medicine 374, 523-532 (2016).

2 Dunlay, S. M., Weston, S. A., Jacobsen, S. J. \& Roger, V. L. Risk factors for heart failure: a population-based case-control study. The American journal of medicine 122, 1023-1028 (2009).

3 Dégano, I. R. et al. The association between education and cardiovascular disease incidence is mediated by hypertension, diabetes, and body mass index. Scientific reports 7, 12370 (2017).

4 Roy, B. et al. Reduced regional cerebral blood flow in patients with heart failure. European journal of heart failure 19, 1294-1302 (2017).

5 Kumar, R. et al. Brain axonal and myelin evaluation in heart failure. Journal of the neurological sciences 307, 106-113 (2011).

6 Kumar, R. et al. Reduced regional brain cortical thickness in patients with heart failure. PloS one 10, e0126595 (2015).

7 Pan, A. et al. Visual assessment of brain magnetic resonance imaging detects injury to cognitive regulatory sites in patients with heart failure. Journal of cardiac failure 19, 94-100 (2013).

8 Woo, M. A. et al. Regional hippocampal damage in heart failure. European journal of heart failure 17, 494-500 (2015).

9 Cermakova, P. et al. Heart failure and dementia: survival in relation to types of heart failure and different dementia disorders. European journal of heart failure 17, 612-619 (2015).

10 Angermann, C. E., Frey, A. \& Ertl, G. (Oxford University Press, 2012).

11 Hajduk, A. M. et al. Cognitive impairment and self-care in heart failure. Clinical epidemiology 5, 407 (2013).

12 Ampadu, J. \& Morley, J. E. Heart failure and cognitive dysfunction. International journal of cardiology 178, 12-23 (2015).

13 Doehner, W. et al. Heart and brain interaction in patients with heart failure: overview and proposal for a taxonomy. A position paper from the Study Group on Heart and Brain Interaction of the Heart Failure Association. European journal of heart failure 20, 199-215 (2018).

14 Pressler, S. J. et al. Cognitive deficits in chronic heart failure. Nursing research 59, 127 (2010).

15 Arnold, J. M. O. et al. Canadian Cardiovascular Society consensus conference recommendations on heart failure 2006: diagnosis and management. Canadian Journal of Cardiology 22, 23-45 (2006).

16 Cleland, J. G. et al. The effect of cardiac resynchronization on morbidity and mortality in heart failure. New England Journal of Medicine 352, 1539-1549 (2005).

17 Frigerio, M. \& Roubina, E. Drugs for left ventricular remodeling in heart failure. The American journal of cardiology 96, 10-18 (2005).

18 Zuccala, G. et al. Use of angiotensin-converting enzyme inhibitors and variations in cognitive performance among patients with heart failure. European heart journal 26, 226-233 (2004).

19 Khachaturian, A. S. et al. Antihypertensive medication use and incident Alzheimer disease: the Cache County Study. Archives of neurology 63, 686-692 (2006).

20 Galli, A. \& Lombardi, F. Neprilysin inhibition for heart failure. The New England journal of medicine 371, 2335, doi:10.1056/NEJMc1412654 (2014). 
21 Solomon, S. D. et al. Angiotensin receptor neprilysin inhibition in heart failure with preserved ejection fraction: rationale and design of the PARAGON-HF trial. JACC: Heart Failure 5, 471-482 (2017).

22 Zannad, F. et al. Rationale and design of a randomized, double-blind, event-driven, multicentre study comparing the efficacy and safety of oral rivaroxaban with placebo for reducing the risk of death, myocardial infarction or stroke in subjects with heart failure and significant coronary artery disease following an exacerbation of heart failure: the COMMANDER HF trial. European journal of heart failure 17, 735-742 (2015).

23 de Bruijn, R. F. \& Ikram, M. A. Cardiovascular risk factors and future risk of Alzheimer's disease. BMC medicine 12, 130 (2014).

24 Wolf, P. A. Contributions of the Framingham Heart Study to stroke and dementia epidemiologic research at 60 years. Archives of neurology 69, 567-571 (2012).

25 Maier, L. The deltaC isoform of CaMKII is activated in cardiac hypertrophy and induces dilated cardiomyopathy and heart failure. Circ Res. 92, 912-919 (2003).

26 Maier, L. S. et al. Transgenic CaMKII $\delta \mathrm{C}$ overexpression uniquely alters cardiac myocyte $\mathrm{Ca} 2+$ handling: reduced SR $\mathrm{Ca} 2+$ load and activated $\mathrm{SR} \mathrm{Ca} 2+$ release. Circulation research 92, 904-911 (2003).

27 Lopez-Crisosto, C. et al. Sarcoplasmic reticulum-mitochondria communication in cardiovascular pathophysiology. Nature Reviews Cardiology 14, 342 (2017).

28 Sweet, M. E. et al. Transcriptome analysis of human heart failure reveals dysregulated cell adhesion in dilated cardiomyopathy and activated immune pathways in ischemic heart failure. BMC genomics 19, 812 (2018).

29 Das, S. et al. Transcriptomics of cardiac biopsies reveals differences in patients with or without diagnostic parameters for heart failure with preserved ejection fraction. Scientific reports 9, 3179 (2019).

$30 \mathrm{Li}$, Y. et al. Cardiac Fibroblast-Specific Activating Transcription Factor 3 Protects Against Heart Failure by Suppressing MAP2K3-p38 Signaling. Circulation 135, 20412057 (2017).

31 Dalkara, T. \& Alarcon-Martinez, L. Cerebral microvascular pericytes and neurogliovascular signaling in health and disease. Brain research 1623, 3-17 (2015).

32 Wendeln, A.-C. et al. Innate immune memory in the brain shapes neurological disease hallmarks. Nature 556, 332 (2018).

33 Erickson, E. K., Farris, S. P., Blednov, Y. A., Mayfield, R. D. \& Harris, R. A. Astrocyte-specific transcriptome responses to chronic ethanol consumption. The pharmacogenomics journal 18, 578 (2018).

34 Rakers, C. et al. Stroke target identification guided by astrocyte transcriptome analysis. Glia 67, 619-633 (2019).

35 Merienne, N. et al. Cell-Type-Specific Gene Expression Profiling in Adult Mouse Brain Reveals Normal and Disease-State Signatures. Cell reports 26, 2477-2493. e2479 (2019).

36 Soto, M., Cai, W., Konishi, M. \& Kahn, C. R. Insulin signaling in the hippocampus and amygdala regulates metabolism and neurobehavior. Proceedings of the National Academy of Sciences 116, 6379-6384 (2019).

37 Hoeffer, C. A. \& Klann, E. mTOR signaling: at the crossroads of plasticity, memory and disease. Trends in neurosciences 33, 67-75 (2010).

38 Marinangeli, C. et al. AMP-activated protein kinase is essential for the maintenance of energy levels during synaptic activation. iScience 9, 1-13 (2018). 
39 Sharma, S. K. \& Carew, T. J. The roles of MAPK cascades in synaptic plasticity and memory in Aplysia: facilitatory effects and inhibitory constraints. Learning \& memory 11, 373-378 (2004).

40 Kerimoglu, C. et al. Histone-methyltransferase MLL2 (KMT2B) is required for memory formation in mice. Journal of Neuroscience 33, 3452-3464 (2013).

41 Hetz, C. \& Papa, F. R. The unfolded protein response and cell fate control. Molecular cell 69, 169-181 (2018).

42 Benito, E. et al. HDAC inhibitor-dependent transcriptome and memory reinstatement in cognitive decline models. The Journal of clinical investigation 125, 3572-3584 (2015).

43 Gjoneska, E. et al. Conserved epigenomic signals in mice and humans reveal immune basis of Alzheimer's disease. Nature 518, 365 (2015).

44 Gispert, S. et al. Potentiation of neurotoxicity in double-mutant mice with Pink1 ablation and A53T-SNCA overexpression. Human molecular genetics 24, 1061-1076 (2014).

45 Swarup, V. et al. Identification of evolutionarily conserved gene networks mediating neurodegenerative dementia. Nature medicine 25, 152 (2019).

46 Kerimoglu, C. et al. KMT2A and KMT2B mediate memory function by affecting distinct genomic regions. Cell reports 20, 538-548 (2017).

47 Stilling, R. M. et al. K-Lysine acetyltransferase 2a regulates a hippocampal gene expression network linked to memory formation. The EMBO journal 33, 1912-1927 (2014).

48 Zhang, T., Cooper, S. \& Brockdorff, N. The interplay of histone modifications-writers that read. EMBO reports 16, 1467-1481 (2015).

49 Jakovcevski, M. et al. Neuronal Kmt2a/Mll1 histone methyltransferase is essential for prefrontal synaptic plasticity and working memory. Journal of Neuroscience 35, 50975108 (2015).

50 Barski, A. et al. High-resolution profiling of histone methylations in the human genome. Cell 129, 823-837 (2007).

51 Bernstein, B. E. et al. Genomic maps and comparative analysis of histone modifications in human and mouse. Cell 120, 169-181 (2005).

52 Vermeulen, M. et al. Selective anchoring of TFIID to nucleosomes by trimethylation of histone H3 lysine 4. Cell 131, 58-69 (2007).

53 Landolin, J. M. et al. Sequence features that drive human promoter function and tissue specificity. Genome research 20, 890-898 (2010).

54 Vecsey, C. G. et al. Histone deacetylase inhibitors enhance memory and synaptic plasticity via CREB: CBP-dependent transcriptional activation. Journal of Neuroscience 27, 6128-6140 (2007).

55 Fischer, A., Sananbenesi, F., Wang, X., Dobbin, M. \& Tsai, L.-H. Recovery of learning and memory is associated with chromatin remodelling. Nature 447, 178 (2007).

56 Nightingale, K. P. et al. Cross-talk between histone modifications in response to histone deacetylase inhibitors MLL4 links histone H3 acetylation and histone H3K4 methylation. Journal of Biological Chemistry 282, 4408-4416 (2007).

57 Rafehi, H. et al. Vascular histone deacetylation by pharmacological HDAC inhibition. Genome research 24, 1271-1284 (2014).

58 Bieberstein, N. I., Oesterreich, F. C., Straube, K. \& Neugebauer, K. M. First exon length controls active chromatin signatures and transcription. Cell reports 2, 62-68 (2012). 
59 Mielcarek, M. et al. SAHA decreases HDAC 2 and 4 levels in vivo and improves molecular phenotypes in the R6/2 mouse model of Huntington's disease. PloS one 6 , e27746 (2011).

60 Hockly, E. et al. Suberoylanilide hydroxamic acid, a histone deacetylase inhibitor, ameliorates motor deficits in a mouse model of Huntington's disease. Proceedings of the National Academy of Sciences 100, 2041-2046 (2003).

61 Reid, A. E. et al. Evaluation of 6-([18F] fluoroacetamido)-1-hexanoicanilide for PET imaging of histone deacetylase in the baboon brain. Nuclear medicine and biology $\mathbf{3 6}$, 247-258 (2009).

62 Yeh, H.-H. et al. Imaging epigenetic regulation by histone deacetylases in the brain using PET/MRI with 18F-FAHA. NeuroImage 64, 630-639 (2013).

63 Zhang, B. et al. Integrated systems approach identifies genetic nodes and networks in late-onset Alzheimer's disease. Cell 153, 707-720 (2013).

64 Miller, J. A., Woltjer, R. L., Goodenbour, J. M., Horvath, S. \& Geschwind, D. H. Genes and pathways underlying regional and cell type changes in Alzheimer's disease. Genome medicine 5, 48 (2013).

65 Parikshak, N. N., Gandal, M. J. \& Geschwind, D. H. Systems biology and gene networks in neurodevelopmental and neurodegenerative disorders. Nature Reviews Genetics 16, 441 (2015).

66 Gandal, M. J. et al. Transcriptome-wide isoform-level dysregulation in ASD, schizophrenia, and bipolar disorder. Science 362, eaat8127 (2018).

67 Langfelder, P. \& Horvath, S. WGCNA: an R package for weighted correlation network analysis. BMC bioinformatics 9, 559 (2008).

68 Pașca, A. M. et al. Human 3D cellular model of hypoxic brain injury of prematurity. Nature medicine 25, 784-791 (2019).

69 Galehdar, Z. et al. Neuronal apoptosis induced by endoplasmic reticulum stress is regulated by ATF4-CHOP-mediated induction of the Bcl-2 homology 3-only member PUMA. Journal of Neuroscience 30, 16938-16948 (2010).

70 Darling, N. J., Balmanno, K. \& Cook, S. J. ERK1/2 signalling protects against apoptosis following endoplasmic reticulum stress but cannot provide long-term protection against BAX/BAK-independent cell death. PloS one 12, e0184907 (2017).

71 Ogata, M. et al. Autophagy is activated for cell survival after endoplasmic reticulum stress. Molecular and cellular biology 26, 9220-9231 (2006).

72 Baumeister, P. et al. Endoplasmic reticulum stress induction of the Grp78/BiP promoter: activating mechanisms mediated by YY1 and its interactive chromatin modifiers. Molecular and cellular biology 25, 4529-4540 (2005).

73 Mathys, H. et al. Single-cell transcriptomic analysis of Alzheimer's disease. Nature, 1 (2019).

74 Hay, M. et al. Cognitive impairment in heart failure: A protective role for angiotensin(1-7). Behavioral neuroscience 131, 99 (2017).

75 de Bruijn, R. F. et al. Subclinical cardiac dysfunction increases the risk of stroke and dementia: the Rotterdam Study. Neurology 84, 833-840 (2015).

76 Rusanen, M. et al. Heart diseases and long-term risk of dementia and Alzheimer's disease: a population-based CAIDE study. Journal of Alzheimer's Disease 42, 183-191 (2014).

77 Qiu, C. et al. Heart failure and risk of dementia and Alzheimer disease: a populationbased cohort study. Archives of internal medicine 166, 1003-1008 (2006).

78 Almeida, O. P.\& Tamai, S. Clinical treatment reverses attentional deficits in congestive heart failure. BMC geriatrics 1, 2 (2001). 
79 Alagiakrishnan, K., Mah, D., Ahmed, A. \& Ezekowitz, J. Cognitive decline in heart failure. Heart failure reviews 21, 661-673 (2016).

80 Čelutkienè, J., Vaitkevičius, A., Jakštienè, S. \& Jatužis, D. Expert opinion-cognitive decline in heart failure: more attention is needed. Cardiac failure review 2, 106 (2016).

81 Hawkins, M. A. et al. The MoCA and MMSE as screeners for cognitive impairment in a heart failure population: a study with comprehensive neuropsychological testing. Heart \& Lung: The Journal of Acute and Critical Care 43, 462-468 (2014).

82 Coughlan, G., Laczó, J., Hort, J., Minihane, A.-M. \& Hornberger, M. Spatial navigation deficits-overlooked cognitive marker for preclinical Alzheimer disease? Nature Reviews Neurology 14, 496 (2018).

$83 \mathrm{Tu}, \mathrm{S}$. et al. Lost in spatial translation-A novel tool to objectively assess spatial disorientation in Alzheimer's disease and frontotemporal dementia. Cortex 67, 83-94 (2015).

84 Virtual reality identifies navigational defects in Alzheimer disease and cognitive aging. Nature Clinical Practice Neurology 4, 638, doi:10.1038/ncpneuro0929 (2008).

85 Cogné, M. et al. The contribution of virtual reality to the diagnosis of spatial navigation disorders and to the study of the role of navigational aids: A systematic literature review. Annals of Physical and Rehabilitation Medicine 60, 164-176 (2017).

86 Hort, J. et al. Spatial navigation deficit in amnestic mild cognitive impairment. Proceedings of the National Academy of Sciences 104, $4042-4047$ (2007).

87 Laczó, J. et al. APOE and spatial navigation in amnestic MCI: Results from a computerbased test. Neuropsychology 28, 676 (2014).

88 Lithfous, S., Dufour, A. \& Després, O. Spatial navigation in normal aging and the prodromal stage of Alzheimer's disease: insights from imaging and behavioral studies. Ageing research reviews 12, 201-213 (2013).

89 Shen, E. Y. et al. Neuronal deletion of Kmt2a/Mll1 histone methyltransferase in ventral striatum is associated with defective spike-timing-dependent striatal synaptic plasticity, altered response to dopaminergic drugs, and increased anxiety. Neuropsychopharmacology 41, 3103 (2016).

90 Webb, W. M. et al. Dynamic association of epigenetic H3K4me3 and DNA 5hmC marks in the dorsal hippocampus and anterior cingulate cortex following reactivation of a fear memory. Neurobiology of learning and memory 142, 66-78 (2017).

91 Gupta, S. et al. Histone methylation regulates memory formation. Journal of Neuroscience 30, 3589-3599 (2010).

92 Cao, D. J. et al. Histone deacetylase (HDAC) inhibitors attenuate cardiac hypertrophy by suppressing autophagy. Proceedings of the National Academy of Sciences 108, 4123-4128 (2011).

$93 \mathrm{Ai}$, S. et al. EED orchestration of heart maturation through interaction with HDACs is H3K27me3-independent. Elife 6, e24570 (2017).

94 He, L. et al. Transcriptional regulator Zeb2 is essential for Bergmann glia development. Journal of Neuroscience 38, 1575-1587 (2018).

95 de Souza Iung, L. H., Mulder, H. A., de Rezende Neves, H. H. \& Carvalheiro, R. Genomic regions underlying uniformity of yearling weight in Nellore cattle evaluated under different response variables. BMC genomics 19, 619 (2018).

96 Mata, A. et al. New functions of Semaphorin 3E and its receptor PlexinD1 during developing and adult hippocampal formation. Scientific reports 8, 1381 (2018).

97 Kuramoto, K., Negishi, M. \& Katoh, H. Regulation of dendrite growth by the Cdc42 activator Zizimin1/Dock9 in hippocampal neurons. Journal of neuroscience research 87, 1794-1805 (2009). 
98 Pathania, M. et al. The autism and schizophrenia associated gene CYFIP1 is critical for the maintenance of dendritic complexity and the stabilization of mature spines. Translational psychiatry 4, e374 (2014).

99 DeLorey, T. et al. Mice lacking the $\beta 3$ subunit of the GABAA receptor have the epilepsy phenotype and many of the behavioral characteristics of Angelman syndrome. Journal of Neuroscience 18, 8505-8514 (1998).

100 Ali, F. \& Meier, R. Primate home range and GRIN2A, a receptor gene involved in neuronal plasticity: implications for the evolution of spatial memory. Genes, Brain and Behavior 8, 435-441 (2009).

101 Bröker-Lai, J. et al. Heteromeric channels formed by TRPC 1, TRPC 4 and TRPC 5 define hippocampal synaptic transmission and working memory. The EMBO journal 36, 2770-2789 (2017).

102 Prut, L. et al. A reduction in hippocampal GABAA receptor $\alpha 5$ subunits disrupts the memory for location of objects in mice. Genes, brain and behavior 9, 478-488 (2010).

103 Wang, S. et al. IQGAP3, a novel effector of Rac1 and Cdc42, regulates neurite outgrowth. J Cell Sci 120, 567-577 (2007).

104 Rizzo, V. et al. Encoding of contextual fear memory requires de novo proteins in the prelimbic cortex. Biological Psychiatry: Cognitive Neuroscience and Neuroimaging 2 , 158-169 (2017).

105 Shen, Y., Fu, W.-Y., Cheng, E. Y., Fu, A. K. \& Ip, N. Y. Melanocortin-4 receptor regulates hippocampal synaptic plasticity through a protein kinase A-dependent mechanism. Journal of Neuroscience 33, 464-472 (2013).

106 Okuyama, T. Social memory engram in the hippocampus. Neuroscience research 129, 17-23 (2018).

107 Kim, H.-N. et al. Genome-wide association study of the five-factor model of personality in young Korean women. Journal of Human Genetics 58, 667 (2013).

108 Schaukowitch, K. et al. An intrinsic transcriptional program underlying synaptic scaling during activity suppression. Cell reports 18, 1512-1526 (2017).

109 Khazaei, M. R. et al. The E3-ubiquitin ligase TRIM2 regulates neuronal polarization. Journal of neurochemistry 117, 29-37 (2011).

110 Takahashi, H. et al. Selective control of inhibitory synapse development by Slitrk3PTP $\delta$ trans-synaptic interaction. Nature neuroscience 15, 389 (2012).

111 Fernandes, C., Hoyle, E., Dempster, E., Schalkwyk, L. \& Collier, D. Performance deficit of $\alpha 7$ nicotinic receptor knockout mice in a delayed matching-to-place task suggests a mild impairment of working/episodic-like memory. Genes, Brain and Behavior 5, 433-440 (2006).

112 Chan, C. S. et al. $\alpha 8$-Integrins are required for hippocampal long-term potentiation but not for hippocampal-dependent learning. Genes, Brain and Behavior 9, 402-410 (2010).

113 Liu, F. et al. Variation in the CACNB2 gene is associated with functional connectivity of the Hippocampus in bipolar disorder. BMC psychiatry 19, 62 (2019).

114 Yook, J. S. et al. Astaxanthin supplementation enhances adult hippocampal neurogenesis and spatial memory in mice. Molecular nutrition \& food research $\mathbf{6 0}$, 589-599 (2016).

115 Tiwari, S. S. et al. Alzheimer-related decrease in CYFIP2 links amyloid production to tau hyperphosphorylation and memory loss. Brain 139, 2751-2765 (2016).

116 Young, J., Meves, J., Tarantino, I., Caldwell, S. \& Geyer, M. Delayed procedural learning in $\alpha 7$-nicotinic acetylcholine receptor knockout mice. Genes, Brain and Behavior 10, 720-733 (2011). 
117 Bouchecareilh, M., Hutt, D. M., Szajner, P., Flotte, T. R. \& Balch, W. E. Histone deacetylase inhibitor (HDACi) suberoylanilide hydroxamic acid (SAHA)-mediated correction of $\alpha 1$-antitrypsin deficiency. Journal of Biological Chemistry 287, 3826538278 (2012).

$118 \mathrm{Wu}, \mathrm{C}$-C. et al. Upregulation of Znf179 acetylation by SAHA protects cells against oxidative stress. Redox biology 19, 74-80 (2018).

119 Byrd, A. E. \& Brewer, J. W. Micro (RNA) managing endoplasmic reticulum stress. IUBMB life 65, 373-381 (2013).

120 Bahari-Javan, S. et al. HDAC1 regulates fear extinction in mice. Journal of Neuroscience 32, 5062-5073 (2012).

121 Sunyer, B., Patil, S., Höger, H. \& Lubec, G. Barnes maze, a useful task to assess spatial reference memory in the mice. Nat Protoc 390, 10-38 (2007).

122 Benito, E., Valor, L. M., Jimenez-Minchan, M., Huber, W. \& Barco, A. cAMP response element-binding protein is a primary hub of activity-driven neuronal gene expression. Journal of neuroscience 31, 18237-18250 (2011). 
A

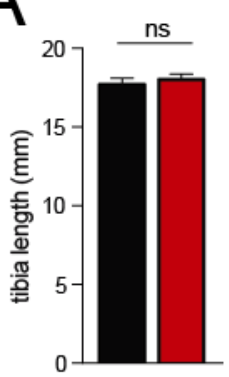

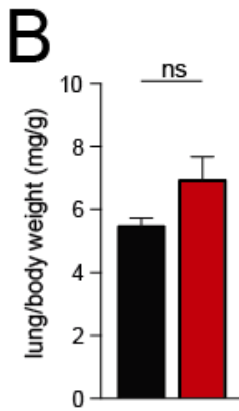

C

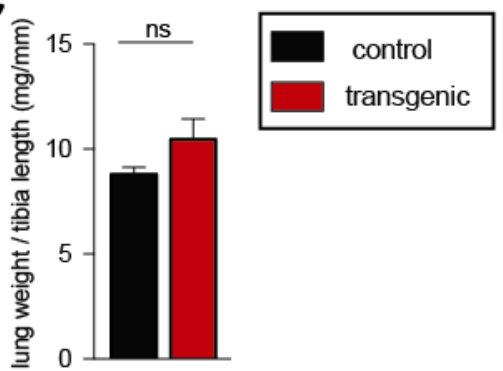

$D$

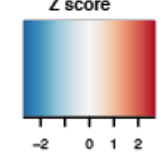

F

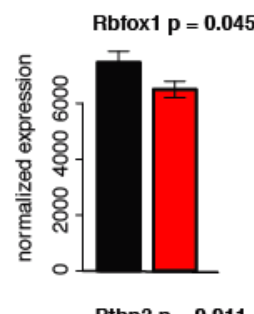

Ptbp3 p $=0.01$



downregulated genes: 1529 upregulated genes: 1562

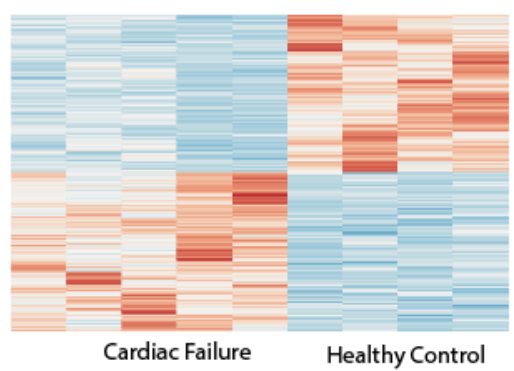

Neuronal splicing Factors

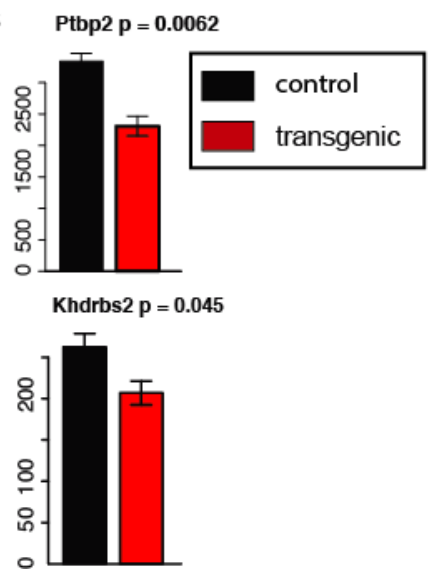

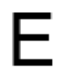



G

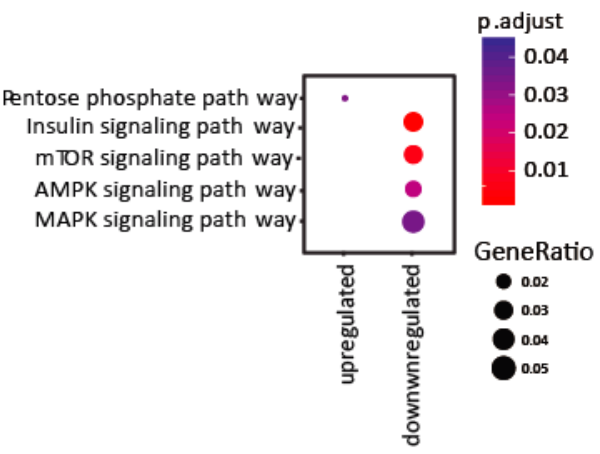

Fig S1. RNA-seq data in transgenic mice.

A-C) Tibia length, lung/body weight ratio, lung weight/tibia length ratio between CamkII $\delta$ c transgenic mice ( $\mathrm{n}=$ 8 ) and littermate controls $(n=5)$. Error bar indicates mean \pm sem. D) Heatmap showing deregulated genes in heart from human patients with heart failure $(n=5)$ compared to healthy controls $(n=4)$. E) Featured biological processes for down-regulated genes. X-axis represents - $\log 10$ (FDR). F) Expression of neuronal splicing factors between control and transgenic mice. Error bar indicates mean \pm sem. G) Dot-plot showing top significant pathways that the deregulated genes may potentially regulate. The color label indicates the level of significance and size of the dot representing the number of deregulated genes involved in the given pathways. 


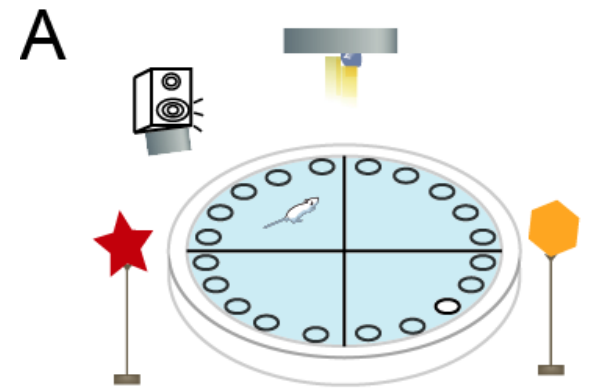

Habituation
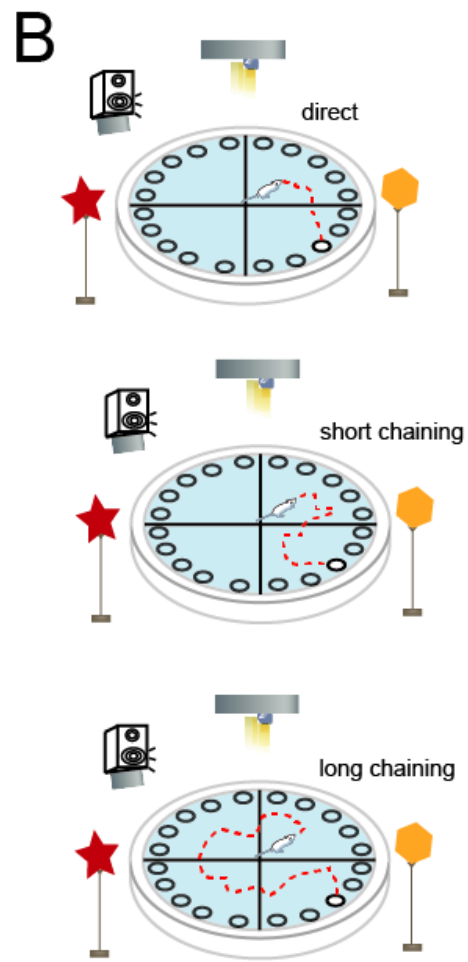

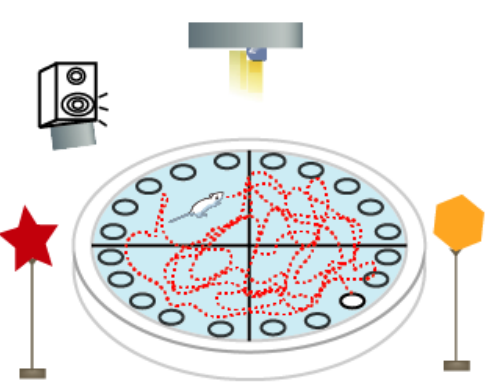

Training Day 1

search strategies


Fig S2. Experimental settings and Different mouse strategies in Barnes Maze.

A) Barnes maze experimental setup is divided into habituation (mice acclimatize with the experimental environment, get exposure to light and sound, perform first experimental task), training trials, and probe test. Initial Training trials help mice learning to locate an escape hole. After several training trials, mice could recall the location of the escape hole during probe test, albeit escape hole is covered during the test. Figure B illustrating different strategic trajectories that mice can employ to locate and then hide in the escape hole, hence the unique name has been assigned to each of these strategies. 


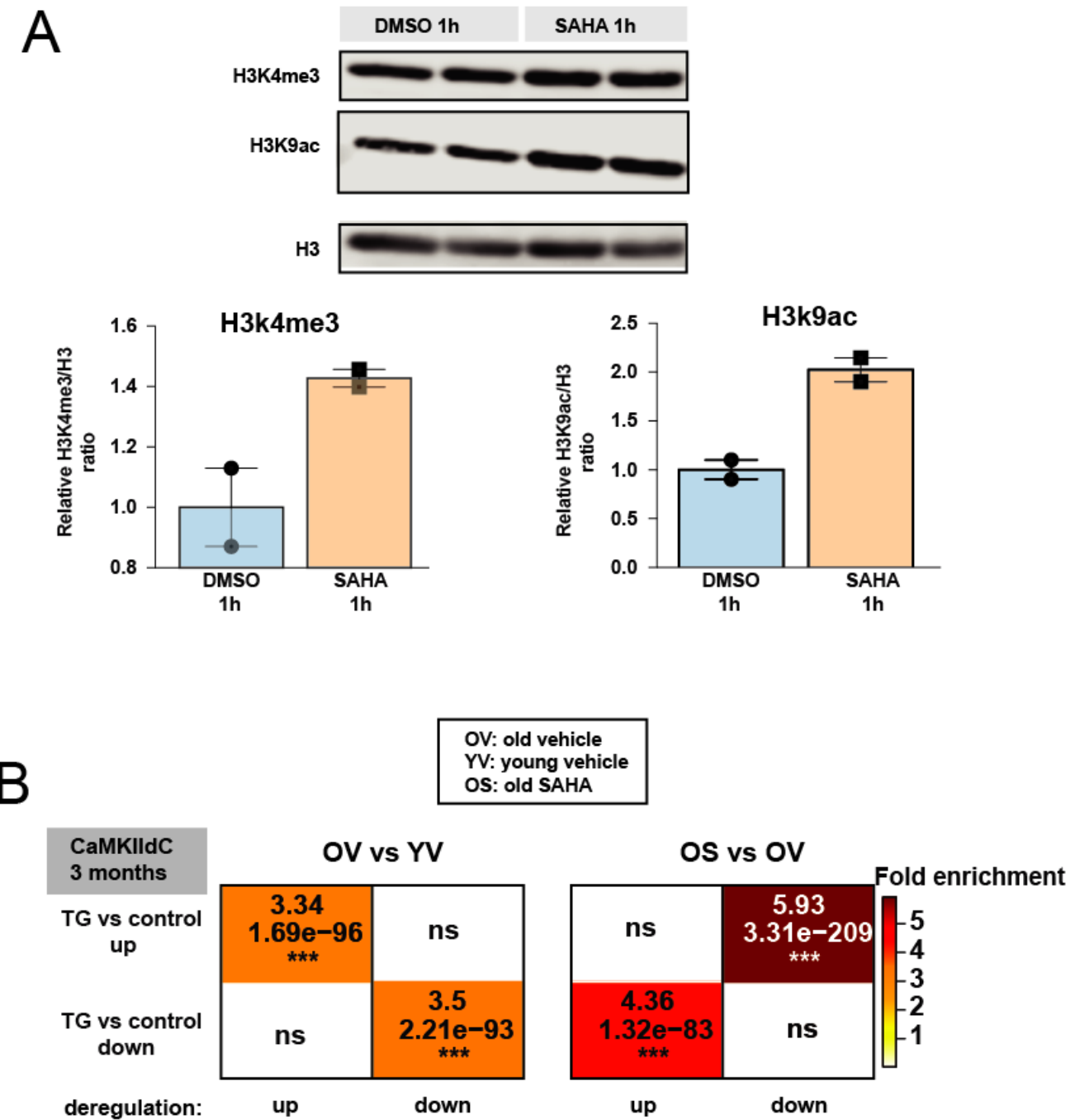

Fig S3. SAHA increases the H3K4me3 level in primary neurons.

A) Western blot for $\mathrm{H} 3 \mathrm{~K} 4 \mathrm{me} 3$ and $\mathrm{H} 3 \mathrm{~K} 9 \mathrm{ac}$ after 1hour of SAHA (1 uM) and DMSO treatment in primary neurons. Two replicates represent pooled samples $(n=3)$ from two independent experiments. Experiment was done at day in vitro 10. Relative Intensity was normalized to H3 level. Error bar indicates mean \pm sem. B) Enrichment analysis of deregulated genes in CamkIISc transgenic with those of old vehicle (OV), young vehicle (YV) and old SAHA (OS) mice. Data are retrieved from Benito et al. 2015. Fold enrichment, significance value, and asterisks (top to bottom) are given in each rectangle corresponding to the comparisons. 



Fig S4. Weighted gene co-expression analysis.

A) Significantly deregulated genes in TV vs. WV and TS vs. WT groups (FDR < 0.05). Up- and down-regulated genes are represented in darkred and darkblue colors respectively B) Venn diagram showing common and uniquely deregulated genes between TV vs. WV and TS vs. WV conditions. C) differential exon usage pattern $($ FDR $<0.05)$ in TV vs. WV and TS vs. WV conditions D) soft power selection based on scale independence and mean connectivity for different modules identification in WGCNA. E) Different modules representing distinct expression patterns among groups. Y-axis representing eigen-expression of the given cluster/module. 


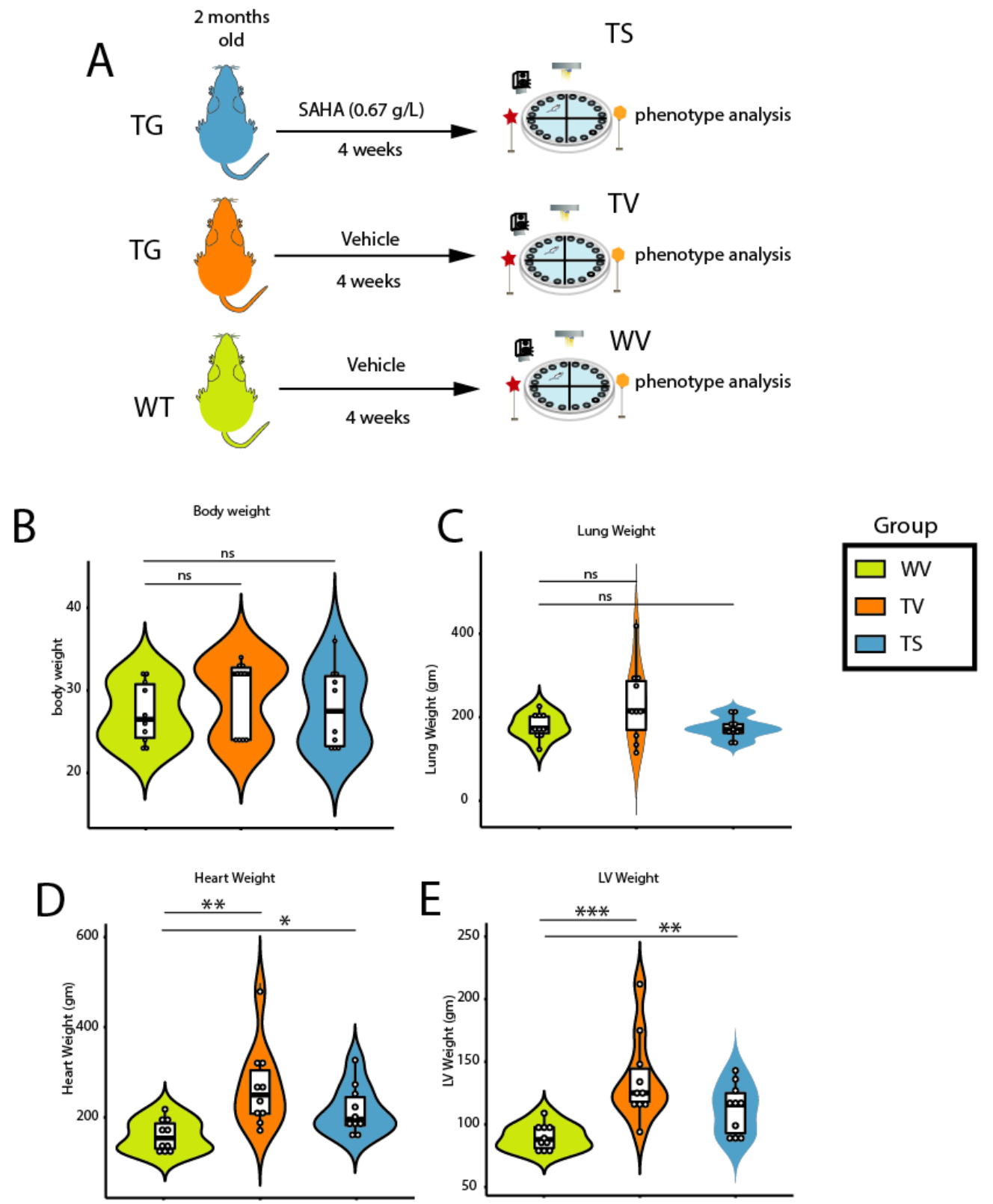

Figure S5. Effect of orally administered SAHA in transgenic mice.

A) Scheme for SAHA or vehicle treatment in transgenic and littermate controls B-C) Body weight and lung weight was similar among groups. D-E) Heart and left ventricular weights increased in both TV and TS groups compared to WV. Violin plot includes densities and box plot. White dot represents each mouse's performance. Kruskal Wallis t-test, ${ }^{*} \mathrm{p}<0.05,{ }^{*} \mathrm{p}<0.01, * * * \mathrm{p}<0.001 . \mathrm{n}=10$ each group. 

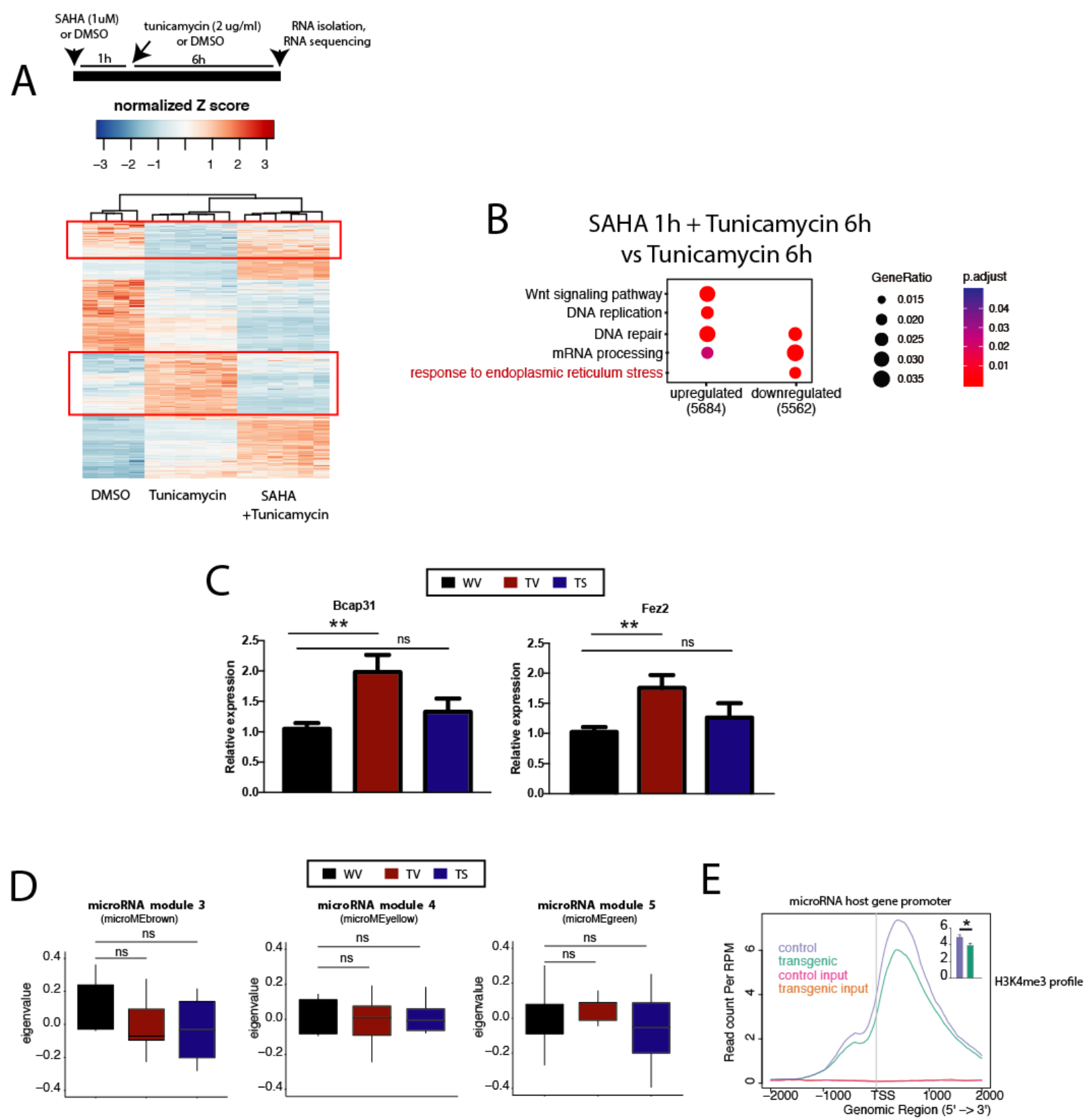

Fig S6. SAHA partially rescues ER stress-related gene expression changes in vitro and in vivo.

A) Scheme for modeling ER stress using tunicamycin $(2 \mathrm{ug} / \mathrm{ml})$ and SAHA mediated rescue experiment. Experiment was performed on day in vitro 10 of primary neuronal culture from the hippocampus. Heatmap showing deregulated genes expression in DMSO, tunicamycin and SAHA pre-treatment + tunicamycin groups. Red rectangular box indicating gene sets that deregulated in response to tunicamycin, are restored in SAHA + tunicamycin group. B) Featured significant GO biological processes. Response to ER response (highlighted in red color) is reduced in SAHA pretreatment group. Dot size represents gene ratio while significance level is represented with color. C) qPCR showing mRNA expression pattern of Bcap31 and Fez2 in three groups. Data normalized to WT vehicle (WV). (WT vehicle: $n=10$, TG vehicle: $n=9$, TG SAHA: $n=8$; multiple t-test, ${ }^{*} p<0.05$, $* * \mathrm{p}<0.001$, error bars indicate mean $\pm \mathrm{sem})$. D) Expression of microRNA modules from WGCNA analysis in three experimental groups. WV: $\mathrm{n}=9$; TV: $\mathrm{n}=7$; TS: $\mathrm{n}=10$; Kruskal Wallis test. E) H3K4me3 profile at promoter of genes that harbor microRNAs. H3K4me3 level at promoter of these genes is significantly reduced in transgenic mice. Unpaired $\mathrm{t}$-test, ${ }^{*} \mathrm{p}<0.05$ 


\section{Discussion on both articles}

Research Article 1 (prepared for submission)

\section{Circulating microRNAs as marker and therapy for}

\section{early cognitive deficits}

Md Rezaul Islam ${ }^{1}$, L. Kaurani ${ }^{2 \$}$, T. Berulava ${ }^{1}$, Urs Heilbronner ${ }^{3}$, Monika Budde ${ }^{3}$, K. Niamkovich $^{3}$, T.P. Centeno ${ }^{1}$, V. Elerdashvilii ${ }^{1}$, E. Benito ${ }^{1}$, P. Rao ${ }^{2}$, C. Kerimoglu ${ }^{1}$, M. Boroomandi $^{1}$, M Sadman Sakib ${ }^{1}$, Fanny Senner ${ }^{3}$, Janos Kalman, J ${ }^{3}$, S. Burkhardt ${ }^{1}$, B. Malchow $^{4}$, H. Bickeboeller ${ }^{5}$, T. Schulze ${ }^{3 *}$, P. Falkai ${ }^{4 *}$, F. Sananbenesi ${ }^{* *}$ A. Fischer ${ }^{1,2^{*}, \#}$

Research Article 2 (prepared for submission)

\section{Epigenetic modifications underpin heart failure associated cognitive deficits}

Md Rezaul Islam ${ }^{1}$, M Sadman Sakib ${ }^{1}$, Raoul Maximilian Hofmann², Dawid Lbik ${ }^{2}$, Tea Berulava ${ }^{1}$, Martí Jiménez Mausbach $^{1}$, Susanne Burkhardt ${ }^{1}$, Alessya Kretzschmar ${ }^{2}$, Julia Cha ${ }^{1}$, Elerdashvili Vakhtang ${ }^{1}$, Dennis M Krueger ${ }^{1}$, Farahnaz Sananbenesi ${ }^{4}$, Karl Toischer ${ }^{2,3}$, Andre Fischer ${ }^{1 *}$ 


\section{Discussion}

Dementia is a slow and progressive neurodegenerative disorder with molecular and metabolic changes taking place years before the onset of disease ${ }^{109}$. With currently available markers and cognitive tests, patients are only diagnosed at an advanced stage of memory decline. As a result, drugs tested so far to improve cognition in demented subjects have been unsuccessful ${ }^{110-112}$. However, an early diagnostic marker will provide opportunities for therapeutic intervention at an earlier stage before substantial neuronal death and brain damage occurs. Moreover, understanding of risk factors and their mechanism to affect cognition will pave the way for deciphering molecular underpinnings at early dementia. Despite high demand, useful markers to diagnose dementia at the pre-clinical stage are still missing. Moreover, the contribution of risk factors at an early stage of memory deficits is poorly understood. Therefore, in this dissertation, I tested the hypothesis that microRNA-based signatures could be potentially informative about early cognitive changes. In a second approach, I investigated the underlying molecular mechanism of cognitive decline due to heart failure (HF), a risk factor of dementia, and explored whether a therapeutic intervention would be able to restore brain functions in HF patients.

In the first study, we identify a blood-based "microRNA signature" consisting of three microRNAs (miR-146a-5p, miR-181a-5p, and miR-148-3p) for diagnosis of early cognitive decline. Co-expression of this signature showed a robust increased pattern in blood at the early onset of cognitive deficits in both healthy mouse and human. Increased co-expression of "microRNA signature" was also observed in the aging brain and that of 4 months old APP/PS1 mice, an Alzheimer's disease (AD) mouse model. Of note, at four months of age, APP/PS1 mice show no overt memory impairment (Agbemenyah et al., unpublished). Interestingly, human MCI patients displayed increased co-expression of this signature compared to age-matched controls. This increment in expression was not limited to human blood but also observed in human cerebrospinal fluid (CSF). In line with these data, we observed increased expression of "microRNA signature" in the brains of various mouse models for memory impairment and AD. This is very interesting, since it indicated that changes in "“"microRNA signature” could inform about relevant pathomechanisms in the CNS. Indeed, when I injected inhibitors of a selected microRNA into the hippocampus of mice suffering from memory impairment, this phenotype was reversed. In summary, our study demonstrates that co-expression of "microRNA signature" in the blood can be sensitive to detect early dementia. 
Although previous studies reported microRNAs-based biomarkers for dementia ${ }^{113-119}$, our "microRNA signature" is different for several reasons. First, previously reported biomarkers were discovered by cross-sectional comparison between healthy and disease conditions and therefore, do not reflect a progressive decline in memory performance ${ }^{113-116,118,119}$. Second, those biomarkers showed high variability in expression depending on its source ${ }^{120,121}$. Third, studies reporting those biomarkers showed conflicting results and were often based on lowquality sequencing data and fewer sample numbers. By contrast, that approach that I adapted is based on the integrative analysis of healthy humans, longitudinal monitoring of changes in cognitive performance in mice, patients, and disease models. Therefore, our signature appears more robust for the early detection of incipient dementia. Consistently, our signature shows similar distinct expression changes across various body fluid sources. Since similar results were observed across several datasets from mouse and human, the "microRNA signature" likely reflects a conserved early pathomechanism in dementia between mice and humans. However, questions remain whether this signature will be able to predict the onset of future dementia. Together with a large longitudinal cohort having well-characterized cognitive information and application of deep learning models on sequencing data, future studies will be able to investigate this.

How can this signature be informative about early cognitive changes? To find an answer, we investigated a recent genome-wide association study (GWAS) and retrieved genes that have been associated with general cognition ${ }^{122}$. Interestingly, target genes of all three microRNAs from our signature significantly overlap with those genes. These data imply that by interacting with crucial memory-related genes, microRNA members of "microRNA signature" may influence learning and memory. Consistently, by modulating one microRNA from signature, miR-181a-5p, we have shown its causal link with memory deficits. However, this result is in sharp contrast to two recent studies showing that miR-181a-5p is important for memory formation ${ }^{123,124}$. This discrepancy could be due to the fact that those two studies investigated contextual fear memory whereas we tested spatial navigation in mice. Unlike fear memories that can be formed without the involvement of the hippocampus, spatial navigation is solely dependent on $\mathrm{it}^{125}$. Therefore, the modulation of microRNA in the dorsal hippocampus might have limited influence in fear memory formation. Moreover, using cell culture model, we found that increased expression of microRNA-181a-5p leads to the down-regulation of cognition related genes, and they are conserved in various neurodegenerative diseases. Therefore, its 
increased expression is likely to be a detriment to cognition as we have observed. Further confidence on this stem from selective inhibition of miR-181a-5p in the dorsal hippocampus. We observed that inhibition of miR-181a-5p could ameliorate cognitive deficits in old mice. Will inhibition of all three microRNAs improve memory as well? Can this inhibition restore memory in Alzheimer's disease? Our preliminary results from mice study demonstrate that inhibition of our signature (all three microRNAs) can ameliorate cognitive deficits in old and APP/PS1 (Alzheimer's mouse model) mice. However, questions remain about its plausible mechanism. Future experiments will address this using high throughput sequencing and imaging technologies.

What can trigger the expression changes of the "microRNA signature"? We reasoned that different risk factors might play a role. To test this conjecture, in the second part of the dissertation, we investigated the role of heart failure (HF) as a risk factor to modulate brain functions. By using a cardiac failure mouse model, we could show that hypoperfusion from heart failure can lead to dramatic gene expression changes in the hippocampus. This alteration in gene expression profile overlapped with that from hypoxic or increased stress condition. Moreover, the changes are in line with findings from a recent study based on single cell RNSseq data from early Alzheimer's disease (AD) ${ }^{126}$. Furthermore, gene expression changes were highly conserved with various neurological diseases. Consistently, HF mice exhibited deficits in both the acquisition and recovery of memory in a spatial learning task. Observed memory deficits were also reported in a previous study after myocardial infarction ${ }^{127}$. By analyzing the distribution of $\mathrm{H} 3 \mathrm{~K} 4 \mathrm{me}$, a histone modification, at the promoter of genes, we could show that a causal relationship exists between H3K4me3 level and reduced expression of cognition related genes. H3K4me3 is essential for maintenance of memory ${ }^{81,128}$, therefore, a decreased level of H3K4me3 may underpin observed cognitive deficits. Growing evidence suggests that human heart failure patients also exhibit memory decline ${ }^{129-133}$. Given that targeted therapy to improve memory in human HF patients is missing ${ }^{134-137}$, we tested SAHA's potential as therapeutic intervention. Oral administration of SAHA increased both cognition related gene expression and cognitive performance in HF mice. Our findings are in line with a previous study showing oral treatment of SAHA provides cognitive benefits to aged and Alzheimer's mouse model $7^{79}$

SAHA could normalize microRNA expression changes in HF mice. Intriguingly, when the expression of the "microRNA signature" from the first part of the dissertation was investigated 
in the HF model, we found an increased expression of the signature in HF mice (Fig T8). This result provides further evidence that the "microRNA signature" could be used as a marker for the diagnosis of early cognitive decline and therapeutic efficacy. These data further imply that hypoperfusion may trigger the changes in expression change of "microRNA signature". Additionally, SAHA could restore expression changes of this "microRNA signature". This data supports our previous experimental results that reduced expression of "microRNA signature" leads to cognitive improvement.

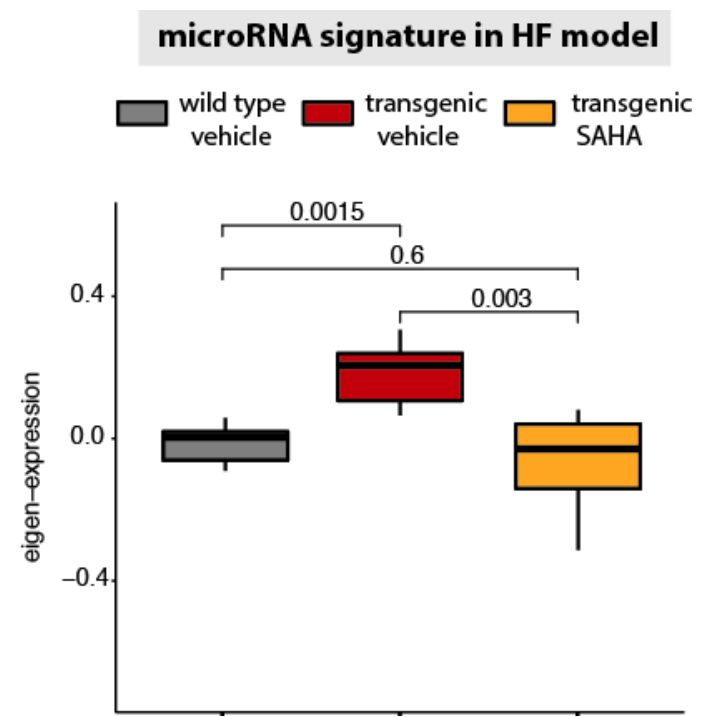

Fig T8. Co-expression analysis of "microRNA signature" in the HF model. Transgenic mice (CamKIIठc) were given SAHA in drinking water for four weeks. Littermate controls and additional transgenic mice groups received drinking water with sugar (vehicle) only. The "microRNA signature" shows increased expression in transgenic vehicle group compared with wild type vehicle. However, Transgenic mice with SAHA treatment show reinstatement in "microRNA signature" expression. n, wild type vehicle:9, transgenic vehicle: 7, transgenic SAHA: 9; Kruskal-Wallis test. Box plot center, lower and upper hinges represent the median, first, and third quartiles, respectively.

In conclusion, the first study reveals potentials of "microRNA signature" as a diagnostic marker for early dementia, and the second study explores pathomechanism of early cognitive deficits due to cardiovascular risk factor and demonstrates the efficacy of SAHA in the cognitive improvement of HF subjects. While future clinical applications for the "microRNA signature" in early risk diagnosis of dementia and SAHA based improved cognition for heart failure patients remain to be tested, these two studies provide critical insights about the marker, molecular underpinnings, and therapy of early cognitive deficits. Further questions remain regarding whether hypoperfusion could be an additional biomarker for accurate detection of early dementia, how the management of cardiovascular risk factors would influence brain function and whether lifestyle and exercise could be a preventive measure for early onset of dementia. 


\section{References}

1 Kandel, E. R. The molecular biology of memory storage: a dialogue between genes and synapses. Science 294, 1030-1038 (2001).

2 Marinesco, S. \& Carew, T. J. Serotonin release evoked by tail nerve stimulation in the CNS of Aplysia: characterization and relationship to heterosynaptic plasticity. Journal of Neuroscience 22, 2299-2312 (2002).

3 Mackey, S., Kandel, E. \& Hawkins, R. Identified serotonergic neurons LCB1 and RCB1 in the cerebral ganglia of Aplysia produce presynaptic facilitation of siphon sensory neurons. Journal of Neuroscience 9, 4227-4235 (1989).

4 Abrams, T. W., Karl, K. A. \& Kandel, E. Biochemical studies of stimulus convergence during classical conditioning in Aplysia: dual regulation of adenylate cyclase by $\mathrm{Ca} 2+/$ calmodulin and transmitter. Journal of Neuroscience 11, 2655-2665 (1991).

5 Klein, M. \& Kandel, E. R. Presynaptic modulation of voltage-dependent Ca2+ current: mechanism for behavioral sensitization in Aplysia californica. Proceedings of the National Academy of Sciences 75, 3512-3516 (1978).

6 Castellucci, V. F. et al. Intracellular injection of the catalytic subunit of cyclic AMPdependent protein kinase simulates facilitation of transmitter release underlying behavioral sensitization in Aplysia. Proceedings of the National Academy of Sciences 77, 7492-7496 (1980).

7 Rescorla, R. A. \& Wagner, A. R. A theory of Pavlovian conditioning: Variations in the effectiveness of reinforcement and nonreinforcement. Classical conditioning II: Current research and theory 2, 64-99 (1972).

8 Ebbinghaus, H. Memory: A contribution to experimental psychology. Annals of neurosciences 20, 155 (2013).

9 Sutton, M. A. \& Carew, T. J. Parallel molecular pathways mediate expression of distinct forms of intermediate-term facilitation at tail sensory-motor synapses in Aplysia. Neuron 26, 219-231 (2000).

10 Schwartz, J. H., Castellucci, V. F. \& Kandel, E. R. Functioning of identified neurons and synapses in abdominal ganglion of Aplysia in absence of protein synthesis. Journal of neurophysiology 34, 939-953 (1971).

11 Roberts, A. C. \& Glanzman, D. L. Learning in Aplysia: looking at synaptic plasticity from both sides. Trends in neurosciences 26, 662-670 (2003).

12 Bacskai, B. J. et al. Spatially resolved dynamics of cAMP and protein kinase A subunits in Aplysia sensory neurons. Science 260, 222-226 (1993).

13 Alberini, C. M., Ghirardl, M., Metz, R. \& Kandel, E. R. C/EBP is an immediate-early gene required for the consolidation of long-term facilitation in Aplysia. Cell 76, 10991114 (1994).

14 Bartsch, D. et al. Enhancement of memory-related long-term facilitation by ApAF, a novel transcription factor that acts downstream from both CREB1 and CREB2. Cell 103, 595-608 (2000).

15 Frey, U. \& Morris, R. G. Synaptic tagging: implications for late maintenance of hippocampal long-term potentiation. Trends in neurosciences 21, 181-188 (1998).

16 Kandel, E. R., Dudai, Y. \& Mayford, M. R. The molecular and systems biology of memory. Cell 157, 163-186 (2014).

$17 \mathrm{Si}$, K. et al. A neuronal isoform of CPEB regulates local protein synthesis and stabilizes synapse-specific long-term facilitation in aplysia. Cell 115, 893-904 (2003).

18 Abel, T., Martin, K. C., Bartsch, D. \& Kandel, E. R. Memory suppressor genes: inhibitory constraints on the storage of long-term memory. Science 279, 338-341 (1998). 
19 Bartsch, D. et al. Aplysia CREB2 represses long-term facilitation: relief of repression converts transient facilitation into long-term functional and structural change. Cell 83, 979-992 (1995).

20 Rajasethupathy, P. et al. A role for neuronal piRNAs in the epigenetic control of memory-related synaptic plasticity. Cell 149, 693-707 (2012).

21 Lee, S.-H. et al. A cellular model of memory reconsolidation involves reactivationinduced destabilization and restabilization at the sensorimotor synapse in Aplysia. Proceedings of the National Academy of Sciences 109, 14200-14205 (2012).

22 Bliss, T. V. \& Lømo, T. Long-lasting potentiation of synaptic transmission in the dentate area of the anaesthetized rabbit following stimulation of the perforant path. The Journal of physiology 232, 331-356 (1973).

23 Morris, R., Anderson, E., Lynch, G. a. \& Baudry, M. Selective impairment of learning and blockade of long-term potentiation by an N-methyl-D-aspartate receptor antagonist, AP5. Nature 319, 774 (1986).

24 Bannerman, D. M. et al. Dissecting spatial knowledge from spatial choice by hippocampal NMDA receptor deletion. Nature neuroscience 15, 1153 (2012).

25 McHugh, T. J. et al. Dentate gyrus NMDA receptors mediate rapid pattern separation in the hippocampal network. Science 317, 94-99 (2007).

26 Nakazawa, K. et al. Requirement for hippocampal CA3 NMDA receptors in associative memory recall. Science 297, 211-218 (2002).

27 Mellor, J. \& Nicoll, R. Hippocampal mossy fiber LTP is independent of postsynaptic calcium. Nature neuroscience 4, 125 (2001).

28 Weisskopf, M. G., Castillo, P. E., Zalutsky, R. A. \& Nicoll, R. A. Mediation of hippocampal mossy fiber long-term potentiation by cyclic AMP. Science 265, 18781882 (1994).

29 Malenka, R. C. \& Bear, M. F. LTP and LTD: an embarrassment of riches. Neuron 44, 5-21 (2004).

30 Awasthi, A. et al. Synaptotagmin-3 drives AMPA receptor endocytosis, depression of synapse strength, and forgetting. Science 363, eaav1483 (2019).

31 Satizabal, C. L. et al. Incidence of dementia over three decades in the Framingham Heart Study. New England Journal of Medicine 374, 523-532 (2016).

32 Dunlay, S. M., Weston, S. A., Jacobsen, S. J. \& Roger, V. L. Risk factors for heart failure: a population-based case-control study. The American journal of medicine 122, 1023-1028 (2009).

33 Dégano, I. R. et al. The association between education and cardiovascular disease incidence is mediated by hypertension, diabetes, and body mass index. Scientific reports 7, 12370 (2017).

34 Cermakova, P. et al. Heart failure and dementia: survival in relation to types of heart failure and different dementia disorders. European journal of heart failure 17, 612-619 (2015).

35 Angermann, C. E., Frey, A. \& Ertl, G. (Oxford University Press, 2012).

36 Dalkara, T. \& Alarcon-Martinez, L. Cerebral microvascular pericytes and neurogliovascular signaling in health and disease. Brain research 1623, 3-17 (2015).

37 Roy, B. et al. Reduced regional cerebral blood flow in patients with heart failure. European journal of heart failure 19, 1294-1302 (2017).

38 Kumar, R. et al. Reduced regional brain cortical thickness in patients with heart failure. PloS one 10, e0126595 (2015).

39 Kumar, R. et al. Brain axonal and myelin evaluation in heart failure. Journal of the neurological sciences 307, 106-113 (2011). 
40 Pan, A. et al. Visual assessment of brain magnetic resonance imaging detects injury to cognitive regulatory sites in patients with heart failure. Journal of cardiac failure 19, 94-100 (2013).

41 Woo, M. A. et al. Regional hippocampal damage in heart failure. European journal of heart failure 17, 494-500 (2015).

42 Hajduk, A. M. et al. Cognitive impairment and self-care in heart failure. Clinical epidemiology 5, 407 (2013).

43 Ampadu, J. \& Morley, J. E. Heart failure and cognitive dysfunction. International journal of cardiology 178, 12-23 (2015).

44 Doehner, W. et al. Heart and brain interaction in patients with heart failure: overview and proposal for a taxonomy. A position paper from the Study Group on Heart and Brain Interaction of the Heart Failure Association. European journal of heart failure 20, 199215 (2018).

45 Pressler, S. J. et al. Cognitive deficits in chronic heart failure. Nursing research 59, 127 (2010).

46 Čelutkienė, J., Vaitkevičius, A., Jakštienė, S. \& Jatužis, D. Expert opinion-cognitive decline in heart failure: more attention is needed. Cardiac failure review 2, 106 (2016).

47 Baquer, N. Z. et al. A metabolic and functional overview of brain aging linked to neurological disorders. Biogerontology 10, 377-413 (2009).

48 Dalton, A. \& Janicki, M. Aging and dementia. Dementia, aging, and intellectual disabilities, 5-39 (1999).

49 Fearnley, J. M. \& Lees, A. J. Ageing and Parkinson's disease: substantia nigra regional selectivity. Brain 114, 2283-2301 (1991).

50 Lindsay, J. et al. Risk factors for Alzheimer's disease: a prospective analysis from the Canadian Study of Health and Aging. American journal of epidemiology 156, 445-453 (2002).

51 Schaie, K. W. Intellectual development in adulthood: The Seattle longitudinal study. (Cambridge University Press, 1996).

52 Hedden, T. \& Gabrieli, J. D. Insights into the ageing mind: a view from cognitive neuroscience. Nature reviews neuroscience 5, 87 (2004).

53 Wimmer, M. E., Hernandez, P. J., Blackwell, J. \& Abel, T. Aging impairs hippocampusdependent long-term memory for object location in mice. Neurobiology of aging 33, 2220-2224 (2012).

54 Zeng, Y. et al. (Soc Neuroscience, 2011).

55 Stilling, R. M. et al. De-regulation of gene expression and alternative splicing affects distinct cellular pathways in the aging hippocampus. Frontiers in cellular neuroscience 8, 373 (2014).

56 van der Heide, L. P., Ramakers, G. M. \& Smidt, M. P. Insulin signaling in the central nervous system: learning to survive. Progress in neurobiology 79, 205-221 (2006).

57 Broughton, S. \& Partridge, L. Insulin/IGF-like signalling, the central nervous system and aging. Biochem. J 418, 1-12 (2009).

58 Yankner, B. A., Lu, T. \& Loerch, P. The aging brain. Annu. Rev. pathmechdis. Mech. Dis. 3, 41-66 (2008).

59 Kaeberlein, M. et al. Regulation of yeast replicative life span by TOR and Sch9 in response to nutrients. Science 310, 1193-1196 (2005).

60 Kapahi, P. et al. Regulation of lifespan in Drosophila by modulation of genes in the TOR signaling pathway. Current Biology 14, 885-890 (2004).

61 Vellai, T. et al. Genetics: influence of TOR kinase on lifespan in C. elegans. Nature 426, 620-620 (2003).

62 Harrison, D. E. et al. Rapamycin fed late in life extends lifespan in genetically heterogeneous mice. nature 460, 392-395 (2009). 
63 Uddin, M. et al. Autophagy and Alzheimer's disease: from molecular mechanisms to therapeutic implications. Frontiers in aging neuroscience 10, 4 (2018).

64 Shehata, M. et al. Autophagy enhances memory erasure through synaptic destabilization. Journal of Neuroscience 38, 3809-3822 (2018).

65 Boisvert, M. M., Erikson, G. A., Shokhirev, M. N. \& Allen, N. J. The aging astrocyte transcriptome from multiple regions of the mouse brain. Cell reports 22, 269-285 (2018).

66 Davie, K. et al. A single-cell transcriptome atlas of the aging Drosophila brain. Cell 174, 982-998. e920 (2018).

67 Cummings, J. The National Institute on Aging-Alzheimer's Association Framework on Alzheimer's disease: Application to clinical trials. Alzheimer's \& Dementia 15, 172178 (2019).

68 Galton, C. J., Patterson, K., Xuereb, J. H. \& Hodges, J. R. Atypical and typical presentations of Alzheimer's disease: a clinical, neuropsychological, neuroimaging and pathological study of 13 cases. Brain 123, 484-498 (2000).

69 Braak, H. \& Del Tredici, K. The preclinical phase of the pathological process underlying sporadic Alzheimer's disease. Brain 138, 2814-2833 (2015).

70 Medina, M. \& Avila, J. New perspectives on the role of tau in Alzheimer's disease. Implications for therapy. Biochemical pharmacology 88, 540-547 (2014).

71 Congdon, E. E. \& Sigurdsson, E. M. Tau-targeting therapies for Alzheimer disease. Nature Reviews Neurology 14, 399 (2018).

72 Beason-Held, L. L. et al. Changes in brain function occur years before the onset of cognitive impairment. Journal of Neuroscience 33, 18008-18014 (2013).

73 Buchhave, P. et al. Cerebrospinal fluid levels of $\beta$-amyloid 1-42, but not of tau, are fully changed already 5 to 10 years before the onset of Alzheimer dementia. Archives of general psychiatry 69, 98-106 (2012).

74 Mehta, D., Jackson, R., Paul, G., Shi, J. \& Sabbagh, M. Why do trials for Alzheimer's disease drugs keep failing? A discontinued drug perspective for 2010-2015. Expert opinion on investigational drugs 26, 735-739 (2017).

75 Coughlan, G., Laczó, J., Hort, J., Minihane, A.-M. \& Hornberger, M. Spatial navigation deficits-overlooked cognitive marker for preclinical Alzheimer disease? Nature Reviews Neurology 14, 496 (2018).

76 Zhang, T., Cooper, S. \& Brockdorff, N. The interplay of histone modifications-writers that read. EMBO reports 16, 1467-1481 (2015).

77 Zhou, V. W., Goren, A. \& Bernstein, B. E. Charting histone modifications and the functional organization of mammalian genomes. Nature Reviews Genetics 12, 7 (2011).

78 Wagner, E. J. \& Carpenter, P. B. Understanding the language of Lys36 methylation at histone H3. Nature reviews Molecular cell biology 13, 115 (2012).

79 Benito, E. et al. HDAC inhibitor-dependent transcriptome and memory reinstatement in cognitive decline models. The Journal of clinical investigation 125, 3572-3584 (2015).

80 Fischer, A., Sananbenesi, F., Wang, X., Dobbin, M. \& Tsai, L.-H. Recovery of learning and memory is associated with chromatin remodelling. Nature 447, 178 (2007).

81 Gupta, S. et al. Histone methylation regulates memory formation. Journal of Neuroscience 30, 3589-3599 (2010).

82 Stilling, R. M. et al. K-Lysine acetyltransferase 2a regulates a hippocampal gene expression network linked to memory formation. The EMBO journal 33, 1912-1927 (2014).

83 Jakovcevski, M. et al. Neuronal Kmt2a/Mll1 histone methyltransferase is essential for prefrontal synaptic plasticity and working memory. Journal of Neuroscience 35, 50975108 (2015). 
84 Kerimoglu, C. et al. KMT2A and KMT2B mediate memory function by affecting distinct genomic regions. Cell reports 20, 538-548 (2017).

85 Großhans, H. \& Filipowicz, W. Molecular biology: the expanding world of small RNAs. Nature 451, 414-416 (2008).

86 Silahtaroglu, A. \& Stenvang, J. MicroRNAs, epigenetics and disease. Essays Biochem 48, 165-185 (2010).

87 Shafi, G., Aliya, N. \& Munshi, A. MicroRNA signatures in neurological disorders. The Canadian Journal of Neurological Sciences 37, 177-185 (2010).

88 Avraham, R. \& Yarden, Y. Regulation of signalling by microRNAs. Biochemical Society Transactions 40, 26 (2012).

89 Bartel, D. P. MicroRNAs: genomics, biogenesis, mechanism, and function. cell 116, 281-297 (2004).

90 Winter, J., Jung, S., Keller, S., Gregory, R. I. \& Diederichs, S. Many roads to maturity: microRNA biogenesis pathways and their regulation. Nature cell biology 11, 228-234 (2009).

91 Rajasethupathy, P. et al. Characterization of small RNAs in Aplysia reveals a role for miR-124 in constraining synaptic plasticity through CREB. Neuron 63, 803-817 (2009).

92 Bredy, T. W., Lin, Q., Wei, W., Baker-Andresen, D. \& Mattick, J. S. MicroRNA regulation of neural plasticity and memory. Neurobiology of learning and memory 96, 89-94 (2011).

93 Konopka, W. et al. MicroRNA loss enhances learning and memory in mice. The Journal of Neuroscience 30, 14835-14842 (2010).

94 Edbauer, D. et al. Regulation of synaptic structure and function by FMRP-associated microRNAs miR-125b and miR-132. Neuron 65, 373-384 (2010).

95 Zovoilis, A. et al. microRNA-34c is a novel target to treat dementias. The EMBO journal 30, 4299-4308 (2011).

96 Gao, J. et al. A novel pathway regulates memory and plasticity via SIRT1 and miR-134. Nature 466, 1105-1109 (2010).

97 Kosaka, N. et al. Trash or Treasure: extracellular microRNAs and cell-to-cell communication. Frontiers in genetics 4 (2013).

98 Shah, M. Y. \& Calin, G. A. The mix of two worlds: non-coding RNAs and hormones. Nucleic acid therapeutics 23, 2-8 (2013).

99 Scott, K. A. et al. Thinking small: towards microRNA-based therapeutics for anxiety disorders. Expert opinion on investigational drugs 24, 529-542 (2015).

100 Li, Y.-J. et al. Alterations of serum levels of BDNF-related miRNAs in patients with depression. PLoS One 8, e63648 (2013).

$101 \mathrm{Hu}, \mathrm{W}$. et al. Functional miRNAs in breast cancer drug resistance. OncoTargets and therapy 11, 1529 (2018).

102 Rao, P., Benito, E. \& Fischer, A. MicroRNAs as biomarkers for CNS disease. Frontiers in molecular neuroscience 6 (2013).

103 Etheridge, A., Lee, I., Hood, L., Galas, D. \& Wang, K. Extracellular microRNA: a new source of biomarkers. Mutation Research/Fundamental and Molecular Mechanisms of Mutagenesis 717, 85-90 (2011).

104 Bouchie, A. First microRNA mimic enters clinic. Nature Biotechnology 31, 577, doi:10.1038/nbt0713-577 (2013).

105 Bader, A. G. miR-34-a microRNA replacement therapy is headed to the clinic. Frontiers in genetics 3, 120 (2012).

106 Chowdhury, S. M. et al. Longitudinal assessment of ultrasound-guided complementary microRNA therapy of hepatocellular carcinoma. Journal of controlled release 281, 1928 (2018). 
107 Acunzo, M., Romano, G., Wernicke, D. \& Croce, C. M. MicroRNA and cancer-a brief overview. Advances in biological regulation 57, 1-9 (2015).

108 Hanna, J., Hossain, G. S. \& Kocerha, J. The Potential for microRNA Therapeutics and Clinical Research. Frontiers in Genetics 10, doi:10.3389/fgene.2019.00478 (2019).

109 Stern, Y. Cognitive reserve. Neuropsychologia 47, 2015-2028 (2009).

110 Mangialasche, F., Solomon, A., Winblad, B., Mecocci, P. \& Kivipelto, M. Alzheimer's disease: clinical trials and drug development. The Lancet Neurology 9, 702-716 (2010).

111 Schneider, L. S. et al. Clinical trials and late-stage drug development for A lzheimer's disease: an appraisal from 1984 to 2014. Journal of internal medicine 275, 251-283 (2014).

112 Abbott, A. \& Dolgin, E. Leading Alzheimer's theory survives drug failure. Nature 540, 15-16 (2016).

113 Chen, R. et al. MicroRNAs as biomarkers of resilience or vulnerability to stress. Neuroscience 305, 36-48 (2015).

114 Leidinger, P. et al. A blood based 12-miRNA signature of Alzheimer disease patients. Genome biology 14, R78 (2013).

115 Galimberti, D. et al. Circulating miRNAs as potential biomarkers in Alzheimer's disease. Journal of Alzheimer's Disease 42, 1261-1267 (2014).

116 Cheng, á. et al. Prognostic serum miRNA biomarkers associated with Alzheimer's disease shows concordance with neuropsychological and neuroimaging assessment. Molecular psychiatry 20, 1188 (2015).

117 Schwarz, E. C. et al. Deep characterization of blood cell miRNomes by NGS. Cellular and Molecular Life Sciences 73, 3169-3181 (2016).

118 Hara, N. et al. Serum microRNA miR-501-3p as a potential biomarker related to the progression of Alzheimer's disease. Acta neuropathologica communications 5, 10 (2017).

119 Nagaraj, S. et al. Profile of 6 microRNA in blood plasma distinguish early stage Alzheimer's disease patients from non-demented subjects. Oncotarget 8, 16122 (2017).

120 Hooten, N. N. et al. Age-related changes in microRNA levels in serum. Aging (Albany NY) 5, 725 (2013).

121 ElSharawy, A. et al. Genome-wide miRNA signatures of human longevity. Aging cell 11, 607-616 (2012).

122 Davies, G. et al. Study of 300,486 individuals identifies 148 independent genetic loci influencing general cognitive function. Nature communications 9, 2098 (2018).

$123 \mathrm{Xu}, \mathrm{X} .-\mathrm{F}$. et al. miR-181a participates in contextual fear memory formation via activating mTOR signaling pathway. Cerebral Cortex 28, 3309-3321 (2017).

124 Zhang, S.-f., Chen, J.-c., Zhang, J. \& Xu, J.-g. miR-181a involves in the hippocampusdependent memory formation via targeting PRKAA1. Scientific reports 7, 8480 (2017).

125 Wiltgen, B. J., Sanders, M. J., Anagnostaras, S. G., Sage, J. R. \& Fanselow, M. S. Context fear learning in the absence of the hippocampus. Journal of Neuroscience 26, 5484-5491 (2006).

126 Mathys, H. et al. Single-cell transcriptomic analysis of Alzheimer's disease. Nature, 1 (2019).

127 Hay, M. et al. Cognitive impairment in heart failure: A protective role for angiotensin(1-7). Behavioral neuroscience 131, 99 (2017).

128 Webb, W. M. et al. Dynamic association of epigenetic H3K4me3 and DNA 5hmC marks in the dorsal hippocampus and anterior cingulate cortex following reactivation of a fear memory. Neurobiology of learning and memory 142, 66-78 (2017).

129 de Bruijn, R. F. et al. Subclinical cardiac dysfunction increases the risk of stroke and dementia: the Rotterdam Study. Neurology 84, 833-840 (2015). 
130 Rusanen, M. et al. Heart diseases and long-term risk of dementia and Alzheimer's disease: a population-based CAIDE study. Journal of Alzheimer's Disease 42, 183-191 (2014).

131 Qiu, C. et al. Heart failure and risk of dementia and Alzheimer disease: a populationbased cohort study. Archives of internal medicine 166, 1003-1008 (2006).

132 Almeida, O. P. \& Tamai, S. Clinical treatment reverses attentional deficits in congestive heart failure. BMC geriatrics 1, 2 (2001).

133 Alagiakrishnan, K., Mah, D., Ahmed, A. \& Ezekowitz, J. Cognitive decline in heart failure. Heart failure reviews 21, 661-673 (2016).

134 Khachaturian, A. S. et al. Antihypertensive medication use and incident Alzheimer disease: the Cache County Study. Archives of neurology 63, 686-692 (2006).

135 Galli, A. \& Lombardi, F. Neprilysin inhibition for heart failure. The New England journal of medicine 371, 2335, doi:10.1056/NEJMc1412654 (2014).

136 Solomon, S. D. et al. Angiotensin receptor neprilysin inhibition in heart failure with preserved ejection fraction: rationale and design of the PARAGON-HF trial. JACC: Heart Failure 5, 471-482 (2017).

137 Zannad, F. et al. Rationale and design of a randomized, double-blind, event-driven, multicentre study comparing the efficacy and safety of oral rivaroxaban with placebo for reducing the risk of death, myocardial infarction or stroke in subjects with heart failure and significant coronary artery disease following an exacerbation of heart failure: the COMMANDER HF trial. European journal of heart failure 17, 735-742 (2015). 


\section{Appendix}

Circulating microRNAs as marker and therapy for early cognitive deficits

Islam and Kaurani et al. 2019

Epigenetic modifications underpin heart failure associated cognitive deficits

Islam et al. 2019

A password protected folder containing all the supplementary files for both articles is accessible through either QR code or direct link as given below

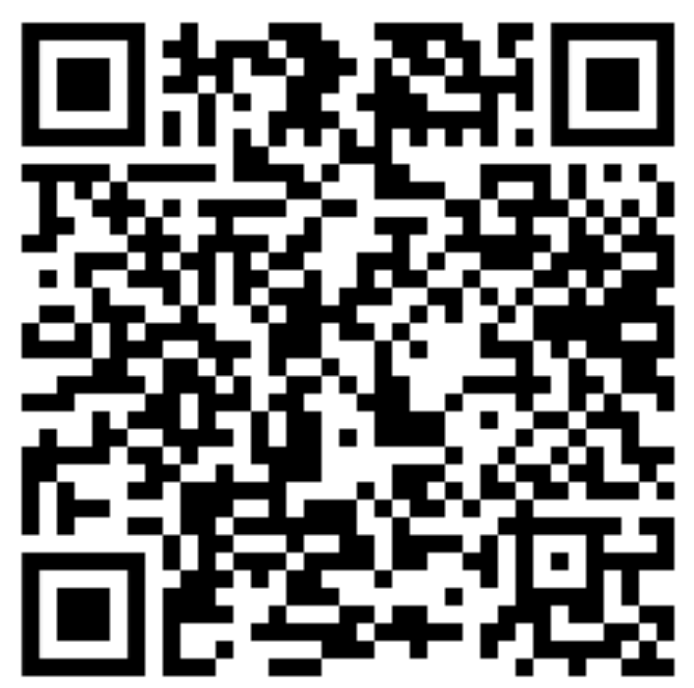

https://docs.google.com/forms/d/e/1FAIpQLSfyT6GLcYI0NhSYKZ2 JHWRnEwEog5BYEJ6-3YmQ5Y15u8Nc3Q/viewform 


\section{Acknowledgements}

I would like to take this unique opportunity to thank my $\mathrm{PhD}$ supervisor Prof. Dr. Andre Fischer for having me in His lab and giving me opportunities to explore different areas of neuroscience. His immense knowledge in the field has always inspired me and His guidance has brought me to this stage. The freedom that He has given me to think of new ideas and to execute those has shaped me completely over the last four years. His continuous motivations kept me moving forward all the time, therefore I cannot believe that I have been in His lab for four years. I enjoyed my time so much that I feel I have been here for only four days. Thank You Andre! I have learnt a lot from You and keep learning.

My thesis would not have been at this stage without close supervision and crucial suggestions that I have received from Prof. Dr. Tiago F. Outeiro and Dr. Camin Dean during our regular meetings. So, I would like to thank them for their time and active participations to guide me for last four years. I would also like to express my gratitude to my extended thesis committee members, Prof. Gregor Eichele, Prof. Ralf Heinrich, and Susann Boretius for their time to take part in my doctoral thesis.

My time in Fischer lab started when I first came to lab to do my Master's thesis. I liked the lab immediately and that is because I was under supervision of an amazing person, Tea Berulava. She not only taught me critical experimental techniques but also motivated me whole time. I found her always there when I had any problems, whether it be personal or professional. It is also because of her, I decided to continue working in this lab. I am grateful to You Tea! I am thankful to Lalit. I learnt about high throughput library preparation from him and later I realized that I also improved my spoken English because of him. Whenever I discussed about science with him, I always found him up to date with recent advancements in the field. His organizing abilities has always inspired me and I want to learn that one day. I would like to thank Sakib. His immense contribution to my project was instrumental and I am grateful to him. I would like to also thank Susi, who keeps helping me with generation of all the sequencing data. Her ability to keep balance between work and personal life is quite motivating for anyone. I would like to thank Vakho, Tonatiuh, Henning, Nicole, Ricardo, Robert, Dennis, Carmelo, Eva, Cemil, Melanie, Sasha, Julia for their help during my time in the lab. I have learnt many things from all of You. I would also like to thank Farah for her continuous supports on my work. I would like to thank my students Luis, Ima, Mariana, and Marti as well for their contribution.

I would thank IMPRS Neuroscience program and its coordination office to give me this opportunity. Many thanks to Sandra for answering all of my questions with prompt reply and being a problem solver.

I would like to express my gratitude to my parents whose constant supports helped me to come to this stage. Although they do not understand in details what my research is about, but they would always push me to go forward. They think that their son is doing something big and I just work for them to make them happy. I thank to Raian and Bushra for loving me so much. I am thankful to my wife Sabah and my little son, Aarham. Without their love, supports, and sacrifices, I would not be here. I would also like to thank the Bangladeshi community in Göttingen for their continuous supports. I thank to my friends and relatives who always wished good things for me, and who did not leave me though I failed to give time to them. Finally, I would like to remember all the others whom I could not mention here but you directly and indirectly helped me a lot to develop my personal and academic life and also contributed significantly to shape my projects. So, a Big Thanks to all of you. 